\title{
Thyroid Hormone Transporters
}

\author{
Stefan Groeneweg, ${ }^{1}$ Ferdy S. van Geest, ${ }^{1}$ Robin P. Peeters, ${ }^{1}$ Heike Heuer, ${ }^{2}$ and \\ W. Edward Visser ${ }^{1}$ \\ ${ }^{1}$ Department of Internal Medicine, Erasmus Medical Center, Rotterdam, the Netherlands Academic Center for \\ Thyroid Diseases, Erasmus Medical Center, 3015 GD Rotterdam, the Netherlands; and 'Department of Endocrinology, \\ Diabetes and Metabolism, University Hospital Essen, University Duisburg-Essen, Essen 45122, Germany \\ ORCiD numbers: 0000-0002-5248-863X (W. E. Visser).
}

ABSTRACT Thyroid hormone transporters at the plasma membrane govern intracellular bioavailability of thyroid hormone. Monocarboxylate transporter (MCT) 8 and MCT10, organic anion transporting polypeptide (OATP) 1C1, and SLC17A4 are currently known as transporters displaying the highest specificity toward thyroid hormones. Structure-function studies using homology modeling and mutational screens have led to better understanding of the molecular basis of thyroid hormone transport. Mutations in MCT8 and in OATP1C1 have been associated with clinical disorders. Different animal models have provided insight into the functional role of thyroid hormone transporters, in particular MCT8. Different treatment strategies for MCT8 deficiency have been explored, of which thyroid hormone analogue therapy is currently applied in patients. Future studies may reveal the identity of as-yet-undiscovered thyroid hormone transporters. Complementary studies employing animal and human models will provide further insight into the role of transporters in health and disease.

(Endocrine Reviews 41: 1 - 55, 2020)

\section{GRAPHICAL ABSTRACT}

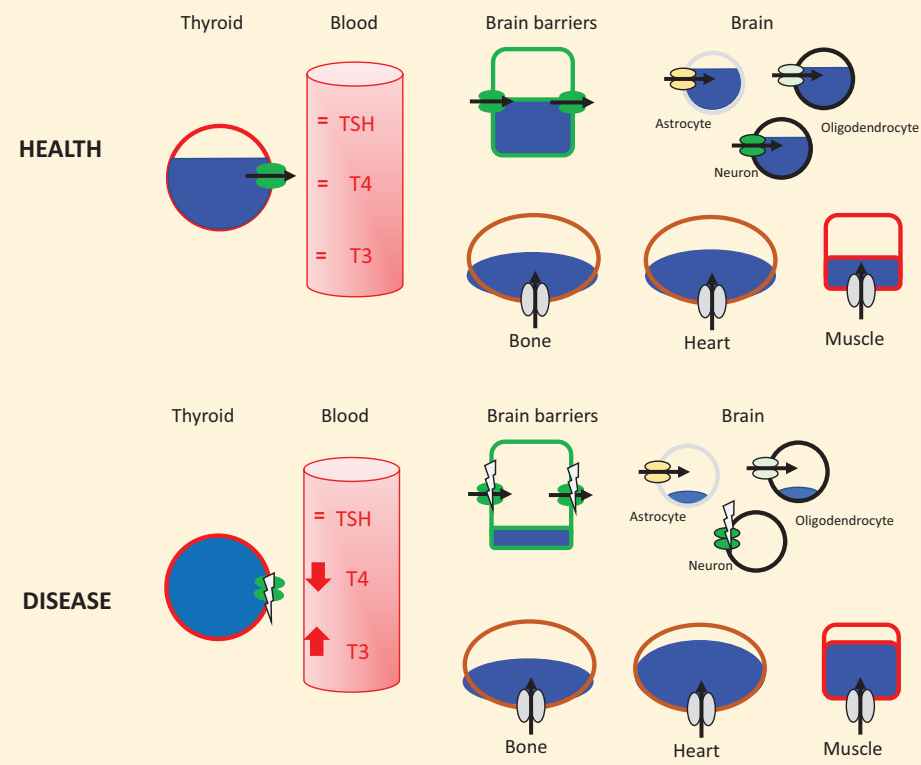

Key Words: thyroid hormone, thyroid hormone transport, thyroid hormone transporters, MCT8, MCT8 deficiency, Allan-Herndon-Dudley syndrome, AHDS, OATP1C1 
ESSENTIAL POINTS

- Thyroid hormones require transporter proteins to facilitate their transport across cell membranes

- Among the up to 16 different thyroid hormone transporters belonging to 5 distinct protein families; monocarboxylate transporter 8 (MCT8) is the most specific thyroid hormone transporter identified to date

- Genetic mutations in MCT8 and organic anion transporting polypeptide (OATP) 1C1 have been associated with clinical syndromes

- MCT8 deficiency (Allan-Herndon-Dudley syndrome) is characterized by a neurocognitive (central) entity related to a hypothyroid state in the brain and a peripheral entity due to T3 excess in the circulation

- Treatment strategies for MCT8 deficiency that are currently being explored include thyroid hormone analogue therapy, (molecular) chaperones, and gene therapy

7 hyroid hormone, the common name for the inactive precursor thyroxine (T4) and the active hormone 3,5,3'-tri-iodothyronine (T3), is important for the development of virtually all tissues and regulation of basal metabolism and tissue regeneration throughout life $(1,2)$.

The genomic effects of thyroid hormone are exerted through binding of T3 to the nuclear T3 receptors (TRs), which are bound to T3-response elements (TREs) in the regulatory regions of $\mathrm{T} 3$ target genes and can act as transcription factors (2). The intracellular T3 concentration is governed by the 3 deiodinating enzymes (DIO1-3) that can either activate or inactivate thyroid hormone, as well as by the activity of plasma membrane transporter proteins that mediate the cellular uptake and/or efflux of T4 and T3 in thyroid hormone target cells (Fig. 1) (3-5). Plasma membrane transporters need to be distinguished from serum thyroid hormonebinding proteins (such as thyroid hormonebinding globulin), which were previously called serum thyroid hormone transporters (6). The transport of thyroid hormones in serum is extensively reviewed elsewhere (6) and falls beyond the scope of this review. There is increased awareness of nongenomic effects of thyroid hormones whose actions involve receptors in the plasma membrane, mitochondria, or cytoplasm (7).

It had been thought for many decades that thyroid hormones could enter their target cells through passive diffusion given the lipophilic nature of iodothyronines. However, accumulating evidence published from the 1970s onward provided evidence that thyroid hormone transfer across the plasma membrane requires a carrier-mediated mechanism, and that the role of passive diffusion, if any, is limited $(8,9)$. These studies indicated that the transport of thyroid hormone into cells is a saturable process, may be inhibited by aromatic and/or aliphatic amino acids, and may depend on $\mathrm{Na}^{+}$in some cell types. Yet, the identity of thyroid hormone plasma membrane transporter proteins started being elucidated in the late 1990s. These early studies, providing the basis for a paradigm shift in the field, have been extensively reviewed in this Journal by Hennemann et al (2001) (8). The turn of the millennium marked the time of several discoveries that had a great impact on the field of thyroidology.

First, several transporters from different protein families were identified at the molecular level as thyroid hormone-transporting proteins. This was a major breakthrough compared to the decades before when only the general characteristics of thyroid hormone transmembrane passage had been studied in nontransfected cells. The most efficient thyroid hormone transporters include monocarboxylate transporter (MCT)8, MCT10, the organic anion transporting polypeptide (OATP)1C1 and, recently, SLC17A4 (10-13).

Another group of discoveries involved the identification of diseases associated with mutations in thyroid hormone transporters. Mutations in MCT8 are associated with severe intellectual disability accompanied by a specific thyroid hormone fingerprint in the blood $(14,15)$. These publications started a new era because they provided ultimate proof for the physiological relevance of thyroid hormone transporters. Recently, the first case of OATP1C1 deficiency has been reported presenting with progressive neurodegeneration and cerebral hypometabolism (16).

In addition, different models have been established to study the role of thyroid hormone transporters in health and disease. Particularly, various experimental models have enlarged the understanding on the (tissue-specific) contribution of various thyroid hormone transporters in the regulation of tissue thyroid hormone state. Novel global and tissue-specific transporter knock-out (ko) mouse models have been generated and characterized over recent years (17-27). In addition, zebrafish and chicken are emerging as complementary vertebrate models to study the role of thyroid hormone transporters. Finally, redifferentiated patient-derived induced 


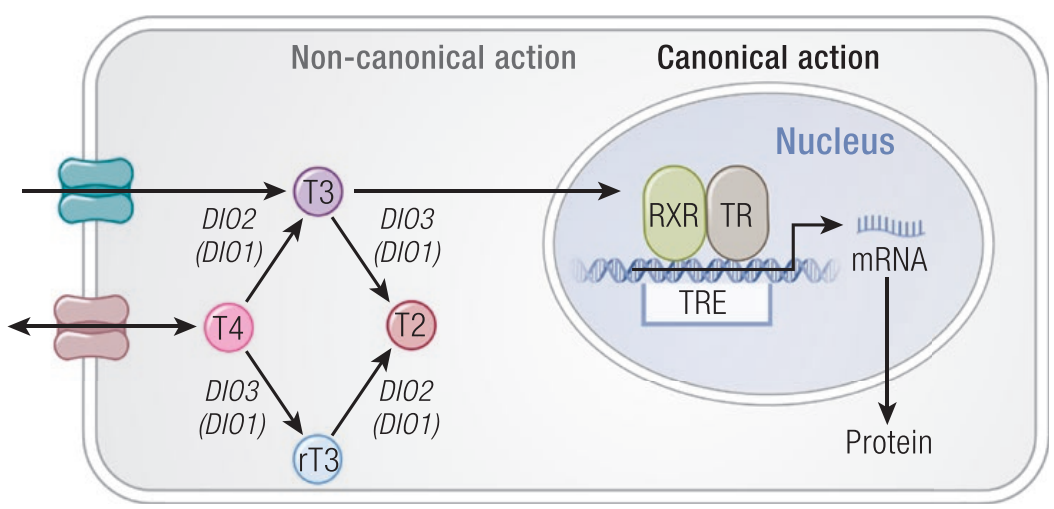

Figure 1. Transport, metabolism, and action of thyroid hormone in a thyroid hormone target cell. Transport across the plasma membrane is facilitated by transporter proteins. Deiodination of thyroid hormones is catalyzed by iodothyronine deiodinases (type 1, D1; type 2, D2; type 3, D3). T3 enters the nucleus, where it binds its receptor (TR) that forms a heterodimer with RXR at T3-responsive elements (TREs) in the promotor regions of $\mathrm{T} 3$ target genes. mRNA indicates messenger RNA.

pluripotent stem cells (iPSCs) have been employed as a human model to understand disease (28-32).

Drug therapy development programs followed the identification of MCT8 deficiency. The abovementioned models largely facilitated testing potential therapeutic intervention for transporter defects. Such preclinical studies indicated that the use of thyroid hormone analogues may hold strong therapeutic potential in MCT8 deficiency, leading to a phase 2 clinical trial on the application of triiodothyroacetic acid (Triac) (33). In addition, chemical chaperones and gene therapy are under active investigation (34-37).

Here we provide an update on thyroid hormone transporter families since 2000, including their functional and molecular characteristics, their contribution to thyroid hormone homeostasis in different tissues, in vivo models to understand the role of transporters, the disorders associated with defects in thyroid hormone transporters, and therapeutic strategies that are currently being investigated.

\section{Thyroid Hormone Transporter Families and Their Molecular Characteristics}

\section{General aspects of thyroid hormone transport}

Thyroid hormone transporters govern the cellular uptake (transport from the extracellular compartment into the cell), the cellular efflux (transport from the cell into the extracellular compartment), or both. To date, about 16 human transporters are considered to transport iodothyronines across the cell membrane. These transporters belong to 5 different protein families, which include the organic anion transporters (OATPs, SLC10, and SLC17),
L-type amino acid transporters (LATs), and monocarboxylate transporters (SLC16 also known as MCTs).

Most thyroid hormone transporters have been identified through in vitro overexpression studies in cell lines or Xenopus oocytes, using $\left[{ }^{125} \mathrm{I}\right]$-radiolabeled iodothyronines to measure cellular thyroid hormone uptake. Such cellular expression systems are indispensable to ensure proper protein conformation and function. The substrate specificity of transporters is generally determined in direct uptake studies with the compounds of interest or indirectly in (cis-)inhibition studies. Cellular thyroid hormone homeostasis in these systems depends not only on the overexpressed transporter of interest, but also on the set of endogenously expressed thyroid hormone transporters. These additional transporter proteins may importantly influence the transport direction (uptake or efflux), as well as the estimates of substrate affinity and transport rate of the transporter being studied. Therefore, reported Michaelis constants (Km values) reflect merely approximate measures for transporter affinity, rather than precise values as calculated in case for purified enzymes. It is important to highlight that some transporters may require specific conditions to function as thyroid hormone transporters. This particularly holds for the so-called secondary active transporters, which couple the uphill transport of thyroid hormone against its concentration or electrochemical gradient to the downhill transport of ions (eg, $\mathrm{Na}^{+}$or $\mathrm{H}^{+}$) along the electrochemical gradient. This is less relevant for transporters that rely on facilitated diffusion, in which case thyroid hormones are transported along their concentration gradient across the cell membrane. By contrast, the presence of competing substrates may reduce the transport of thyroid hormones. Application of the appropriate and, moreover, physiologically relevant conditions 
is thus imperative to assess whether a transporter accepts thyroid hormones as a substrate.

This section provides an overview of the thyroid hormone transporters that have been identified to date, with a particular focus on those that are considered to transport thyroid hormones in vivo. Their main characteristics will be summarized and discussed, including phylogenetics, substrate specificity, and transport direction, as well as their tissue distribution and regulation of expression.

\section{Organic anion transporting polypeptides}

Phylogenesis of the organic anion transporting polypeptide superfamily Organic anion transporting polypeptides (OATPs) form a gene superfamily with more than 300 members across at least 40 different species, together classified as the solute carrier family SLCO for gene classification and OATP for the corresponding protein nomenclature (38). The OATP superfamily represents a large group of homologous proteins that accept a wide range of substrates, including anionic, but also neutral and even cationic compounds. Over the years, the nomenclature of the OATP members has been subjected to changes that pose considerable confusion in literature. To date, up to 40 different OATPs have been identified in humans, rats and mice, forming 6 major families (OATP1, OATP2, OATP3, OATP4, OATP5, and OATP6) that can be further categorized into several subfamilies (eg, OATP1A, OATP1B, and OATP1C) based on their amino acid sequence identities (38, 39). Within these subfamilies the individual OATPs are numbered according to the chronology of their discovery, and the same number is given to any orthologs. It should be noted that the organization of some of these (sub)families is very different in humans from that in mice and rats because some members have not been conserved among species $(38,39)$.

In humans, 11 different OATPs have been identified, of which 8 are capable of transporting iodothyronines in vitro (Fig. 2). Four of these genes, encoding OATP1A2, 1B1, 1B3, and $1 \mathrm{C} 1$, are clustered together on human chromosome 12p12 (Table 1).

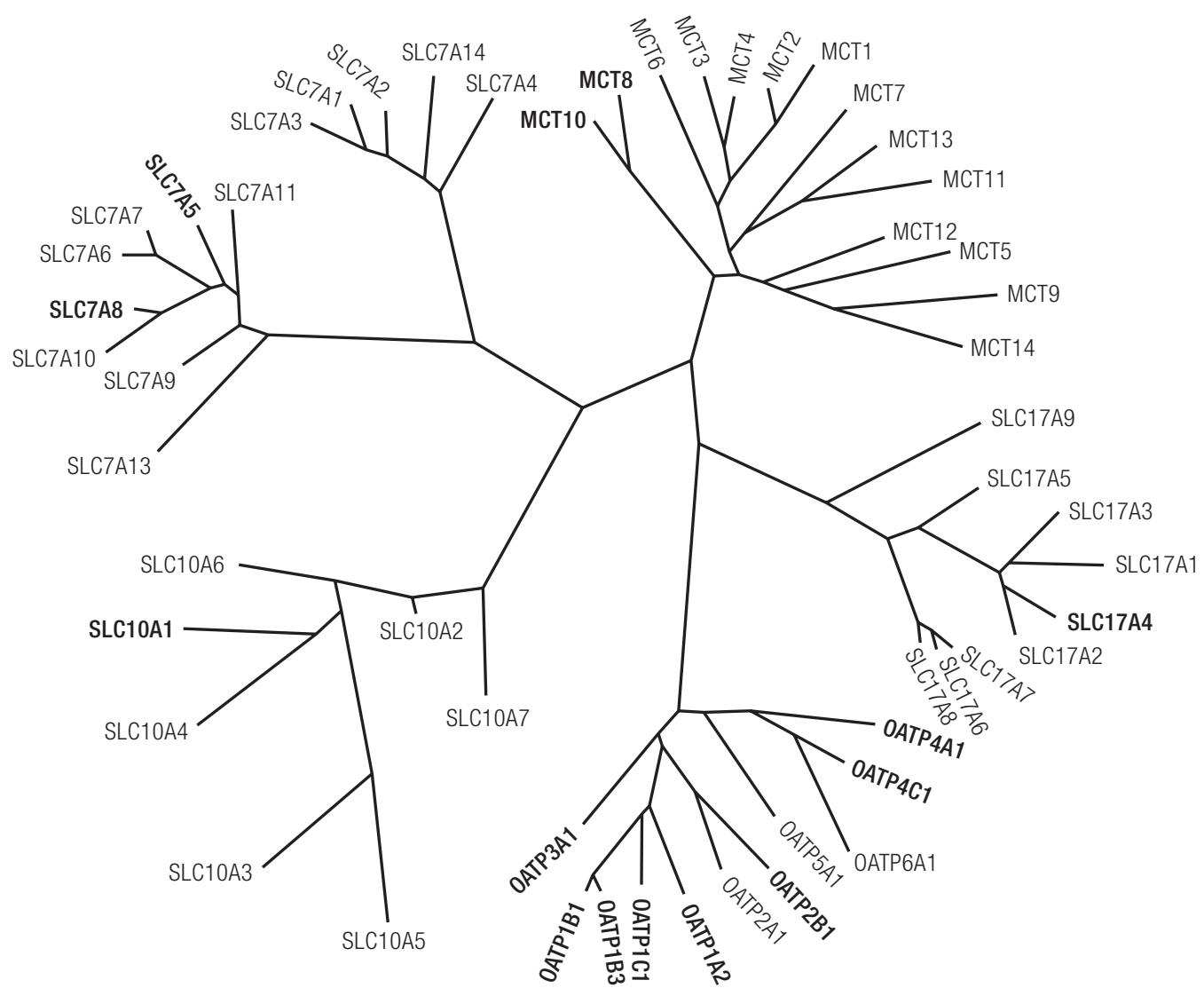

Figure 2. Phylogenetic tree including the protein families to which the currently known human thyroid hormone transporters belong. Phylogenetic trees have been generated using MUSCLE multiple sequence alignment of ENSEMBL reference protein sequences and the PhyML algorithm available at http://www.phylogeny.fr/alacarte.cgi. The thyroid hormone transporting (human) members of each transporter family are indicated in bold. 


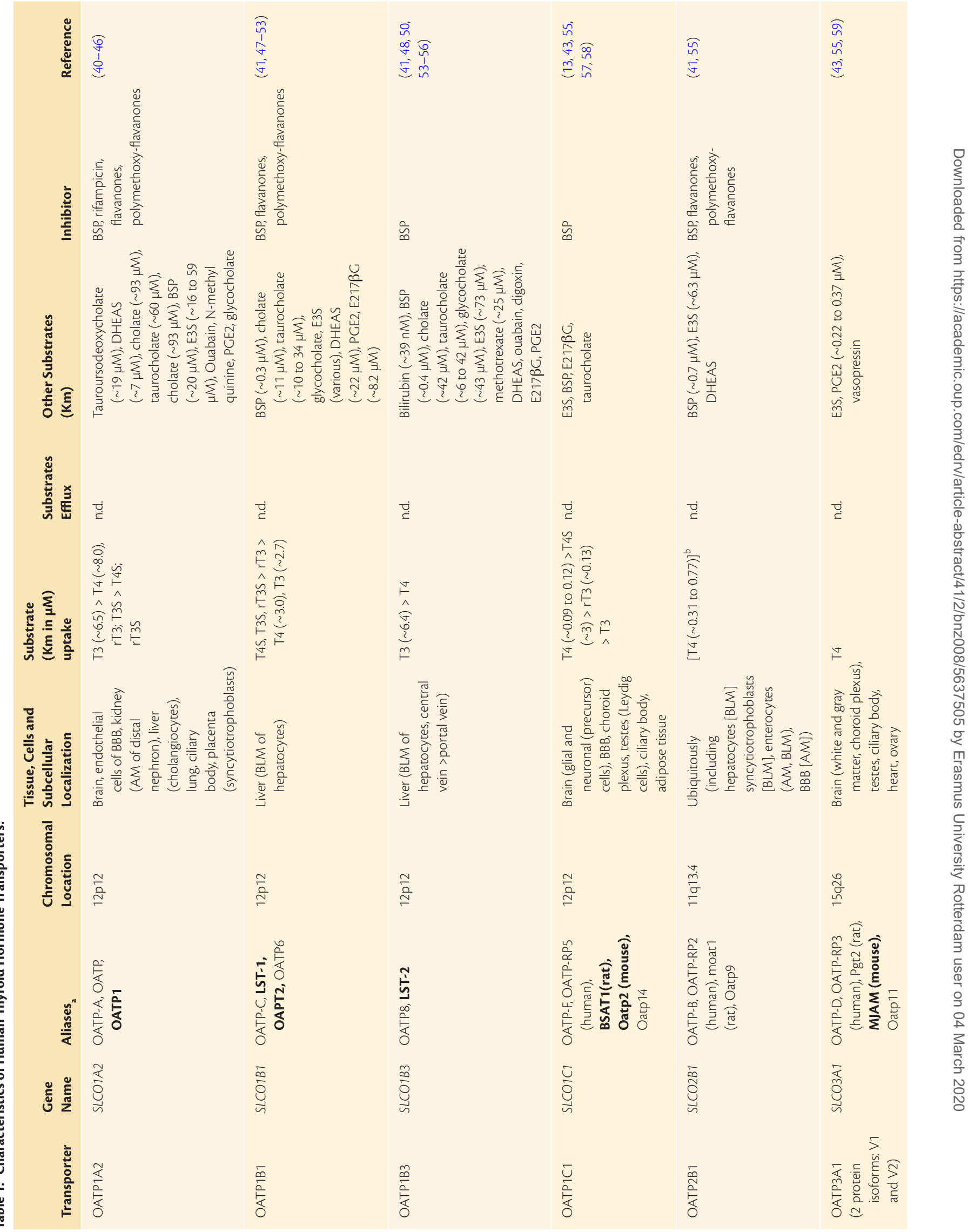




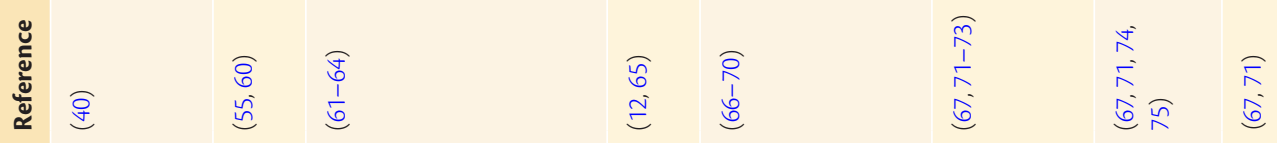
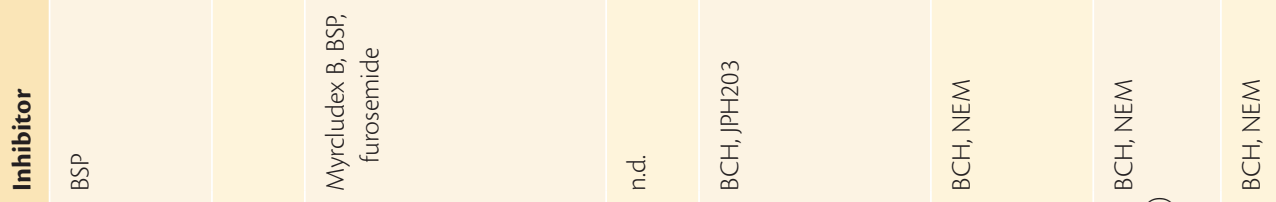

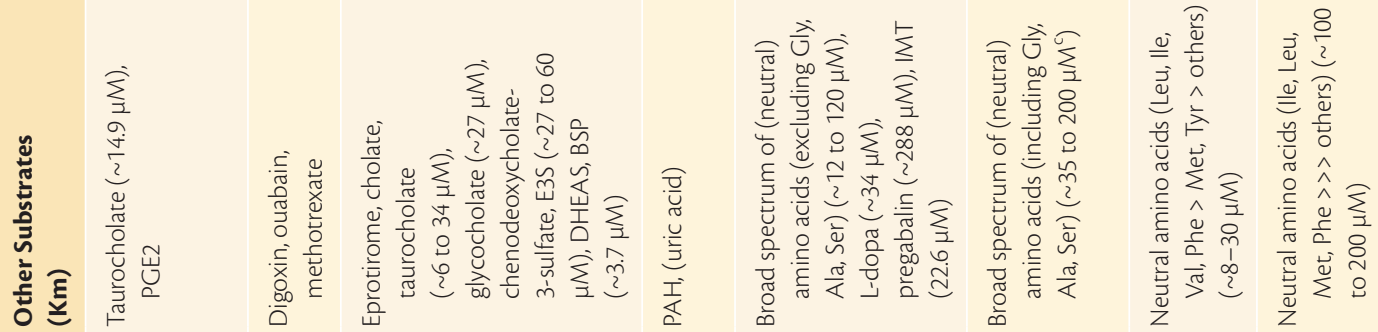

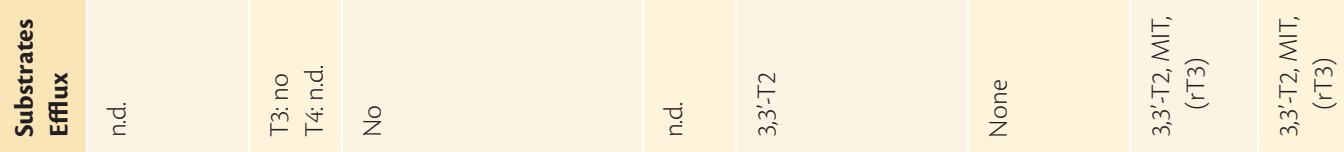

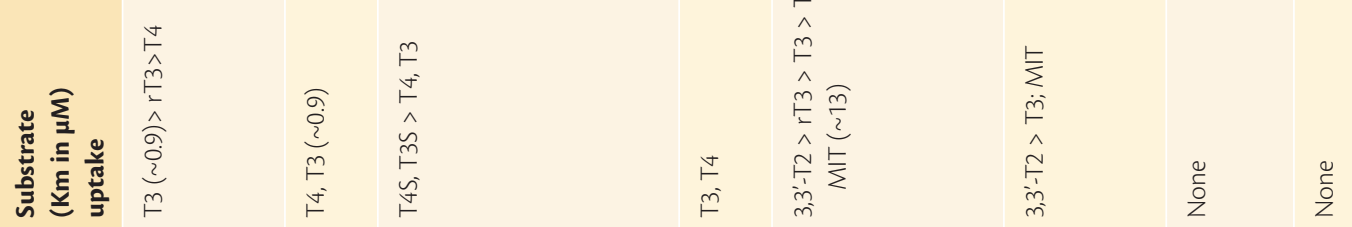

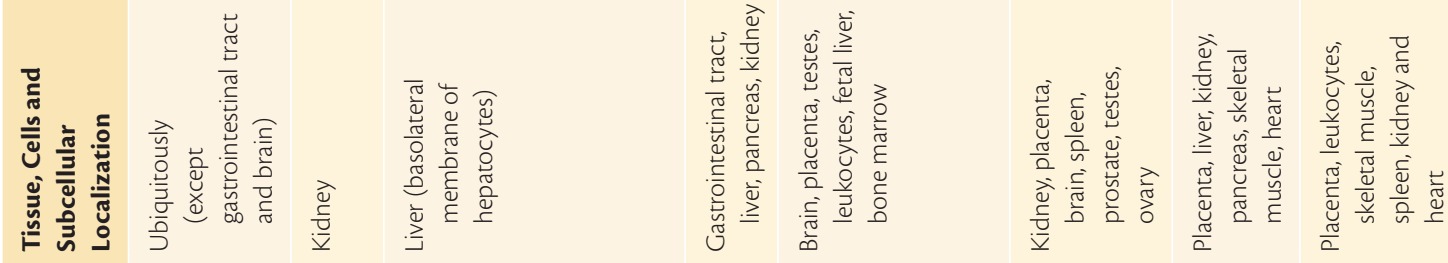

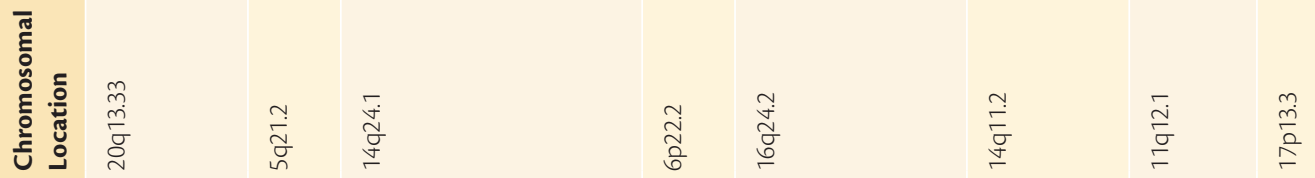

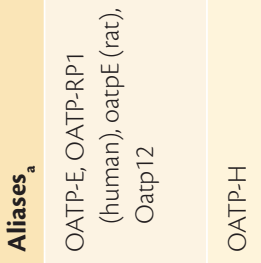

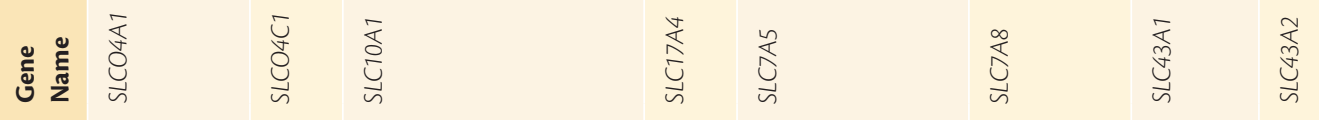

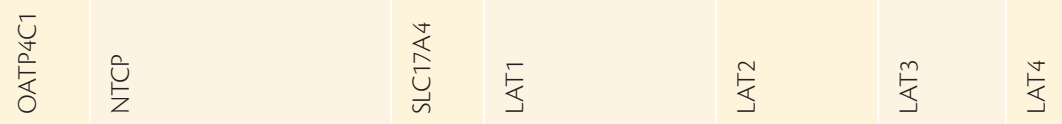




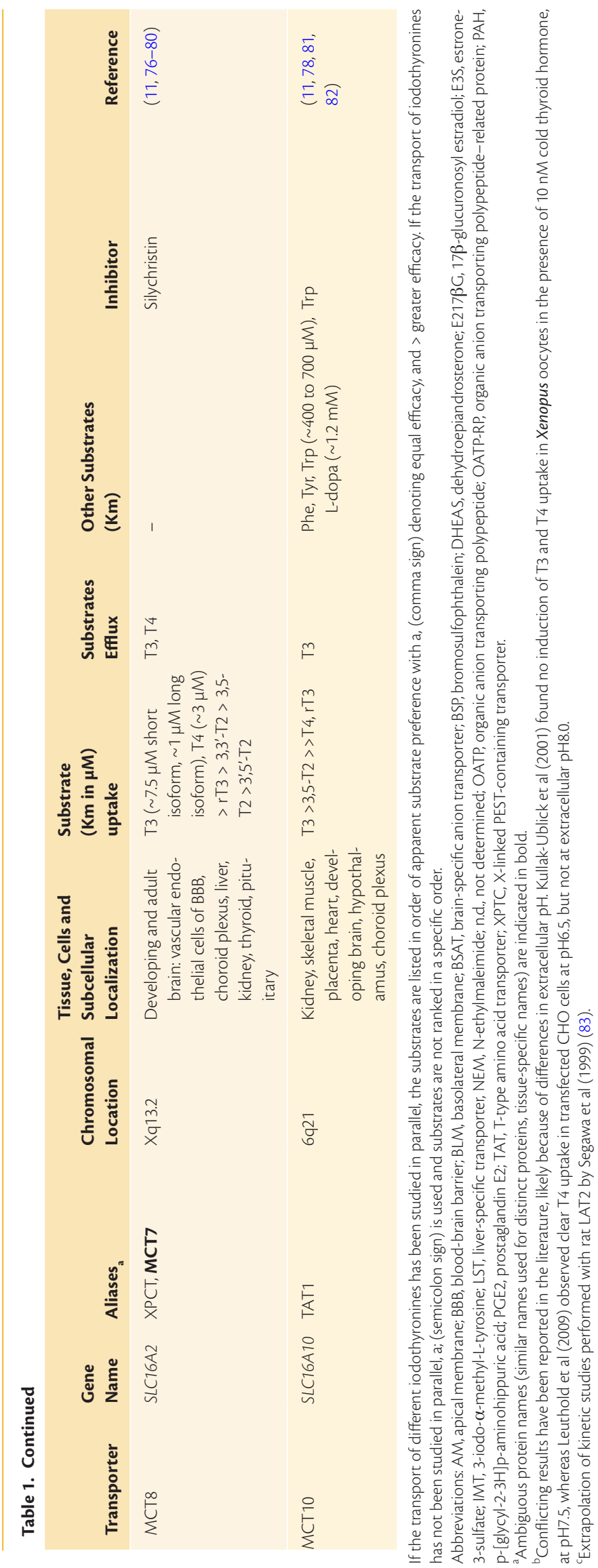


Substrate specificity and transport direction Rat OATP1A1 was the first member of the SLCO superfamily to be isolated (84) and was initially characterized as a sodium-independent bile acid transporter with a preference for unconjugated (eg, cholate) over conjugated (eg, taurocholate) bile acids (84-86). Soon afterward, rat OATP1A1 was among the first transporters shown to transport iodothyronines (87). Following this observation, OATP1A2 was the first human OATP that was found to transport several iodothyronines (rT3, T3, and T4) $(40,41)$ and their sulfoconjugates (42), as well as a broad spectrum of other substrates, including conjugated and unconjugated bile acids, bromosulfophthalein (BSP), and dehydroepiandrosterone sulfate (Table 1$)(88,89)$.

In the subsequent years, additional OATPs have been cloned and functionally evaluated. These studies revealed that most human OATPs are multispecific transporters that accept a broad range of substrates, including several bile acids, steroid hormones and conjugates thereof, linear and cyclic peptides, prostaglandins, and multiple drugs and other xenobiotics (reviewed in $(38,90)$ ), some of which are shown in Table 1. In addition to OATP1A2, several other members of the OATP family were found to facilitate the uptake of iodothyronines or metabolites thereof, including OATP1B1 (47-49), OATP1B3 (42, 54), OATP1C1 (13, 57), OATP2B1 (55), OATP3A1 (55, 59), OATP4A1 (40), and OATP4C1 $(38,60,91)$. Besides rat OATP1A1, rat OATP1A3 $(48,92)$, OATP1A4 (48), OATP1A5 (48), OATP1B1 (93), OATP1B2 (94), OATP1B3 (93), OATP1C1 (95), OATP4A1 (40), OATP4C1 (60), OATP6B1 (96), and OATP6C1 (96) transport iodothyronines, each with its unique substrate preferences and affinities (summarized in (38, 91)). Many of these OATPs show a relatively low affinity toward iodothyronines and transport a multitude of other substrates for these transporters (Table 1). For these reasons, it is currently unclear whether all OATPs that were found to transport thyroid hormone in vitro also exert meaningful contributions to cellular thyroid hormone homeostasis in vivo. OATP1C1 is currently considered the only member of the OATP family that contributes to thyroid hormone transport in vivo, and therefore the remainder of this section will focus particularly on this transporter.

Compared to other OATPs, OATP1C1 has the highest affinity for and greatest specificity toward iodothyronines, with apparent $\mathrm{Km}$ values of 90.4 $\mathrm{nM}$ for T4, and 127.7 nM for rT3, whereas its affinity for $\mathrm{T} 3$ seems considerably lower (13, 57). OATP1C1 also facilitates the uptake of sulfoconjugated T4 (T4S) and enhances its intracellular metabolism by DIO1 (57). The uptake of T4 by OATP1C1 exhibits a biphasic kinetic profile, suggesting the presence of a low- and a highaffinity binding site (97). Moreover, rat OATP1C1 facilitates the efflux of T4 in transfected human embryonic kidney 293 cells (95), indicating that OATP1C1 may also be involved in the cellular efflux of T4.

Although the exact transport mechanism of OATP1C1 is not fully understood, OATPs are known to act as organic anion exchangers (98) and are therefore classified as secondary active transporters. Multiple counterions have been shown to potentiate OATP-mediated transport, the characteristics of which have been mostly studied on substrates other than thyroid hormones (extensively reviewed by Hagenbuch and Stieger (38)), but the preferred counterions for OATP1C1-mediated T4 transport are currently unknown. The uptake by various OATPs is stimulated by a low extracellular $\mathrm{pH}$, with the exception of OATP1C1 $(55,99)$. Therefore, it appears imperative to study thyroid hormone uptake by OATPs other than OATP1C1 at physiologically relevant extracellular $\mathrm{pH}$ to determine their relative substrate preferences (55).

Several (cis-side) inhibitors, such as rifampicin, gemfibrozil, and BSP, have been used to study the contribution of OATPs, including OATP1C1, to overall thyroid hormone transport in cell systems. However, the inhibiting effects of most of these compounds are not completely specific for OATPs and their potency may vary between substrates (reviewed in more detail in (38)). Recent work has demonstrated that several components of grapefruit and orange juice, including different flavanones (eg, naringing and naringenin) and polymethoxyflavanones (eg, nobiletin and tangeretin) inhibit members of the OATP family, including OATP1A2 and OATP2B1 (100-103). It is yet unclear whether these compounds also inhibit OATP1C1-mediated T4 transport and whether they should be regarded as (specific) OATP-inhibitors.

Taken together, most of the human OATPs have thus been shown to transport thyroid hormones in an in vitro setting, but only OATP1C1 appears to have a relatively high substrate specificity toward T4 and has been shown to function as a thyroid hormone transporter in vivo (see "Mechanisms of Disease-Monocarboxylate Transporter 8 and Organic Anion Transporting Polypeptide-1C1 Deficiency in Human and Animal Models").

Tissue distribution and regulation of expression OATPs are expressed in virtually all tissues. Some members, such as OATP2B1, OATP3A1, and OATP4A1, are expressed ubiquitously, whereas the 
expression pattern of others, including OATP1B1, OATP1B3, and OATP1C1, is more restricted (50). Especially at the messenger RNA (mRNA) level, most OATPs are widely expressed, although the tissue expression profile has not always been confirmed at the protein level because of lack of suitable antibodies. An overview of the tissue distribution of the thyroid hormone-transporting
OATP members at the mRNA level in humans is presented in Fig. 3 and Table 1. Considering the overlapping tissue distribution of many thyroid hormone-transporting OATPs, the precise impact of single transporter deficits is difficult to study in vivo. So far, alterations in tissue thyroid hormone homeostasis have been observed only in mice deficient in OATP1C1 (see "OATP1C1"). Therefore,
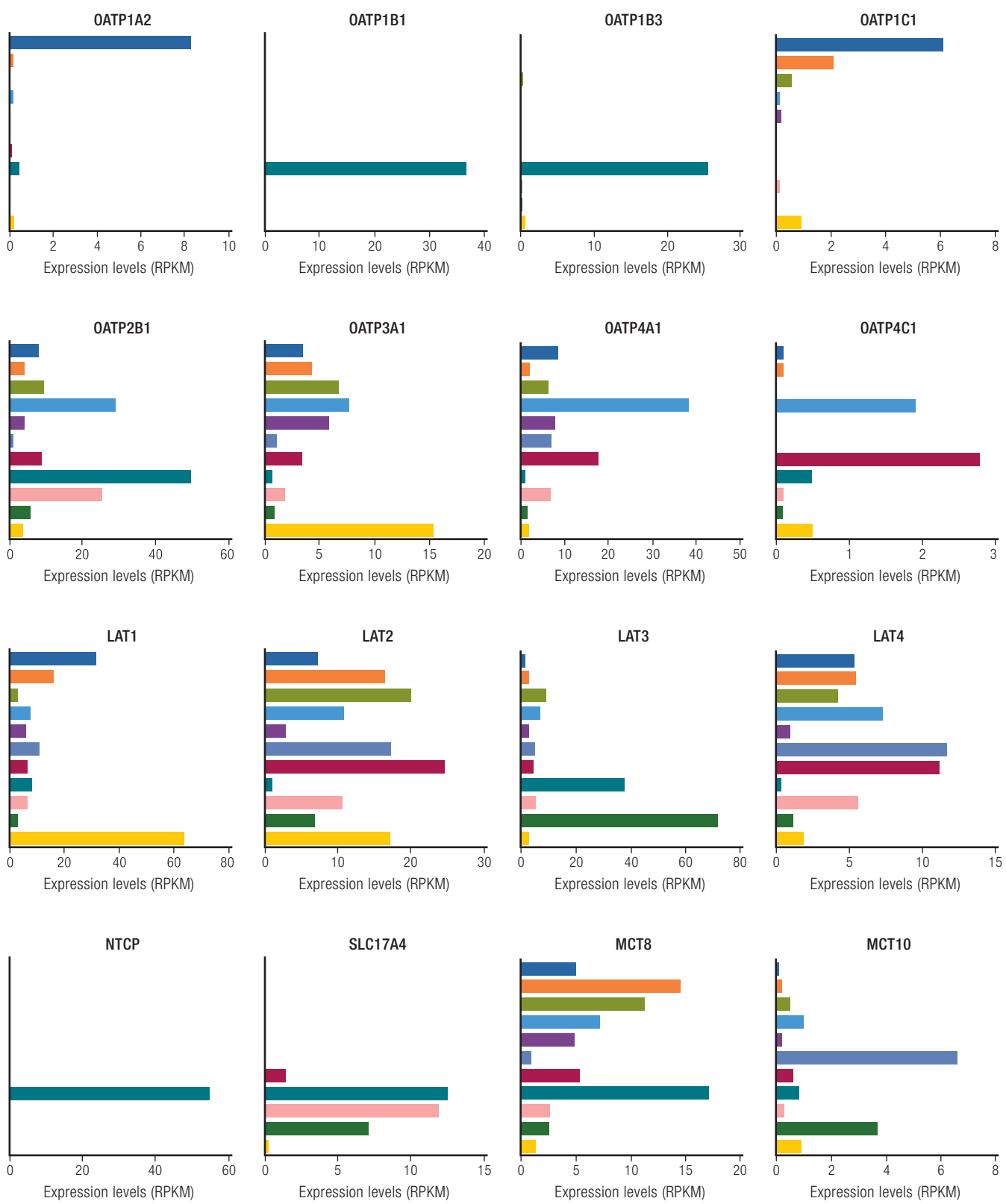

\begin{tabular}{|llll|}
\hline$\square$ Cerebral cortex & $\square$ Lung & Kidney & Pancreas \\
$\square$ Pituitary & $\square$ Heart & $\square$ Liver & Testis \\
$\square$ Thyroid & $\square$ Skeletal muscle & $\square$ Small intestine & \\
\hline
\end{tabular}

Figure 3. Tissue distributions of human thyroid hormone transporters. Expression profiles are based on messenger RNA levels retrieved from the Genotype-tissue-expression (GTEx) RNA sequencing project, accessible at https://www.proteinatlas.org/, and reflect expression levels in adult tissues. 
we will here detail only the tissue distribution and transcriptional regulation of OATP1C1. For a detailed overview of the tissue distribution of other human OATPs, we refer to an extensive review by Hagenbuch and Stieger (38).

OATP1C1 is considered a brain-specific transporter, although a limited number of other tissues also express OATP1C1. In humans, these tissues include Leydig cells of the testes (13) and the ciliary body of the eye $(43,104)$. OATP1C1 mRNA is widely abundant in the human brain, with the highest expression in the cerebral cortex, amygdala, caudate nucleus, hippocampus, and putamen, but not in the pons or cerebellum (13). Recent studies have detailed the cell-specific distribution of OATP1C1 in the developing human brain from gestational week (GW) 14 to GW38 (58). Throughout development, OATP1C1 protein was abundantly present in epithelial cells of the choroid plexus, which together with stromal vessels constitute the blood-cerebrospinal fluid barrier (BCSFB). OATP1C1 expression was also found in the leptomeningeal cells and blood vessels in the subarachnoid space, which together comprise the outer cerebrospinal fluid brain barrier (CSFBB), as well as in ependymocytes and tanycytes, which constitute the inner CSFBB. Only weak OATP1C1 expression was detected in the capillary vessels of the blood-brain barrier (BBB), although from GW32 onward OATP1C1 immunoreactivity was detected in astrocytes surrounding these vessels. In radial glial cells, OATP1C1 protein was detected from the apical neuroepithelial surface in the ventricular zone to the cortical surface, although the intensity differed along the trajectory. Other cell types inside the brain were also found to express OATP1C1 protein, including immature neurons and Cajal-Retzius cells. Human control samples lacking MCT8 were available in these studies, which was not the case for OATP1C1, DIO2, or DIO3.

In humans, OATP1C1 thus seems to be present at different brain barriers, except for the BBB, as well as in different thyroid hormone target cells inside the brain. The contributions of the various barriers to the total thyroid hormone transport into the brain and the role of OATP1C1 therein are currently unknown, as is further discussed in "Mechanisms of Disease-Monocarboxylate Transporter 8 and Organic Anion Transporting Polypeptide-1C1 Deficiency in Human and Animal Models."

In rodents OATP1C1 is also predominantly expressed in the brain and only a few other tissues, including the villous interstitial substance in the placenta (105) and different structures of the eye (43, 104). In the adult rodent brain, Oatp1c1 is localized in distinct subsets of astrocytes and tanycytes as well as the basolateral membrane of epithelial cells in the choroid plexus. In sharp contrast to the human situation, OATP1C1 is also present at the luminal and abluminal membrane in vascular endothelial cells of the $\mathrm{BBB}$ in the rodent central nervous system (CNS) $(18,95,106-108)$. Further, oatp1c1 expression can be detected at the mRNA level in vascular structures within the brain of zebrafish (29) but not in the monkey (109). These observations indicate an important species difference in the localization of OATP1C1 in primates vs rodents and fish, as discussed in more detail in "Mechanisms of Disease-Monocarboxylate Transporter 8 and Organic Anion Transporting Polypeptide-1C1 Deficiency in Human and Animal Models."

A growing number of studies have reported on the transcriptional regulation of OATPs which generally involves tissue-specific factors, growth factors, cytokines, and chemicals (reviewed in (38)). Oatp1c1 transcript levels in rat capillary endothelial cells were found to be affected by the thyroidal state because higher expression was found in hypothyroid animals and reduced expression in hyperthyroid animals $(95,110)$. Similarly, the expression levels of Oatp1c1 increased in the fetal part of the placenta during maternal hypothyroidism in the rat (105). The mechanism by which OATP1C1 is regulated by thyroid hormone is currently unknown, and it is unclear whether OATP1C1 is a direct target for the T3 receptors. Given the low apparent Km of OATP1C1 for T4, it is conceivable that relatively small fluctuations in expression levels may influence T4 transport. Other regulating factors are currently poorly defined, although a striking reduction in Oatp1c1 expression has been observed in rodent brain capillary cells during inflammation (111).

\section{Sodium/taurocholate cotransporting polypeptide}

Phylogenesis of the SLC10A family The SLC10 family comprises 7 established members that are uptake transporters of bile acid, steroidal hormones, specific drugs, and a variety of other substrates (Fig. 2) (112). They have long been regarded as the sodium bile salt cotransporting family because the first 2 members, sodium/taurocholate cotransporting polypeptide (NTCP; SLC10A1) and the apical sodium-dependent bile acid transporter (ASBT; SLC10A2), are primarily bile salt transporters (113). However, later studies revealed that the other members of the family, SLC10A3 to 7, transport a great variety of 
other substrates, and in some cases no bile acids. This prompted further evaluation of NTCP, which turned out to transport substrates other than bile salts, including thyroid hormone (Table 1). Despite thorough evaluation, NTCP is the only member of the SLC10 family that accepts thyroid hormone as a substrate (61). The NTCP amino acid sequence is well conserved across species, and orthologs have been identified in fish and amphibians (62).

Substrate specificity and transport direction NTCP was isolated by expression cloning from rat liver mRNA $(114,115)$ and found to mediate the sodium-coupled uptake of taurocholate and other bile acids (116). Similar to its rat ortholog, human NTCP transports all physiological bile salts and their conjugates when expressed in oocytes and various cell systems (117), with reported apparent affinities in the low micromolar range (1-30 $\mu \mathrm{M})$ (Table 1) (reviewed in (62)). In addition, NTCP transports bile acids, sulfoconjugated steroids such as estron-3-sulfate (E3S), and cholephilic compounds such as BSP with similar affinity, the latter 2 being frequently used as competitive (though nonselective) inhibitors of NTCPmediated transport (62). On overexpression in Xenopus oocytes, rat NTCP induced the uptake of T3 and T4 by about 2-times over uninjected control oocytes, whereas the influx of rT3 was induced by 6 times (87). In addition, rat NTCP facilitates the uptake of the sulfoconjugated iodothyronines T3S and T4S (87). Also, human NTCP facilitates the sodium-dependent uptake of T4 and to a lesser extent T3, and, even more efficiently, their respective sulfoconjugates in overexpressing mammalian cell lines (61). The uptake of T3S and T4S was largely inhibited in presence of $50 \mu \mathrm{M}$ taurocholate, suggesting that transport of thyroid hormones by NTCP can be impeded if bile acid concentrations rise (eg, after a meal) (61). Overexpression of NTCP induces T4S and to a lesser extent T3S metabolism by the DIO1 (61). In accordance with the unidirectional transport of bile acids, NTCP does not facilitate the efflux of T3S or T4S (61). In addition to endogenous (sulfoconjugated) iodothyronines, NTCP facilitates the uptake of the synthetic thyromimetic drug eprotirome (63).

Recent studies suggested that NTCP is involved in the infection of liver cells with hepatitis B virus and hepatitis D virus, which could be selectively inhibited by the synthetic peptide Myrcludex B (118). This molecule also inhibits bile acid transport by NTCP $(64,119)$ and is currently applied in clinical trials (120). Further studies should reveal whether this molecule also selectively inhibits
NTCP-mediated thyroid hormone transport in the liver, which may provide an additional tool to define the contribution of NTCP to thyroid hormone homeostasis in vivo.

Tissue distribution and regulation of expression In rodents, NTCP is exclusively expressed in the liver, where it is localized at the basolateral membrane of differentiated hepatocytes $(121,122)$. During rat development, Ntcp mRNA can be first detected between days 18 and 21 of gestation (123), although its expression levels remain low during fetal development. At birth, rodent Ntcp expression levels strongly increase $(123,124)$. Similar to rodents, human NTCP is specifically expressed in the liver (Fig. 3) (125). Expression levels are relatively low during fetal development, possibly contributing to the high $\mathrm{T} 3 \mathrm{~S}$ and $\mathrm{T} 4 \mathrm{~S}$ concentrations at that stage, and about 20 times higher in adult liver (126). Expression of NTCP is regulated by several liganddependent transcription factors (eg, retinoic acid receptor [RAR] a, glucocorticoid receptor) and several hepatic transcription factors (reviewed in detail in (127)). It is currently unknown whether thyroid hormone-related factors drive the expression of NTCP in the liver.

Given its broad substrate specificity and high affinity for alternative substrates, it is tempting to speculate that NTCP functions as a general hepatic anion transporter in the liver rather than a specific thyroid hormone transporter.

\section{SLC17A4}

Phylogenesis of the SLC17 family The SLC17 family is a group of 9 structurally related proteins that have been identified as organic anion transporters (Fig. 2) (128). The first 4 members (SLC17A1-4) were termed type I phosphate transporters (NPTs) following the classification of SLC17A1 as an $\mathrm{Na}^{+}$dependent inorganic phosphate transporter (129, 130). Other mammalian members that have been identified are the lysosomal acidic sugar transporter sialin (SLC17A5), the vesicular glutamate transporters (VGLUT1-3: SLC17A7, SLC17A6, and SLC17A8 respectively), and the vesicular nucleotide transporter (VNUT; SLC17A9). These proteins are integral membrane transporters that reside on the plasma membrane (SLC17A1-4), lysosomes (SLC17A5), or synaptic vesicles (SLC17A6-9).

At present, SLC17A4 is the only member of the SLC17 family that has been demonstrated to transport thyroid hormones (12), although it should be noted that other members of this family have not 
been tested thus far. From an evolutionary perspective, SLC17A1-4 appear to be the most recent members of the SLC17 family, and are present only in mammals (131). In human, the genes encoding SLC17A1-4 are all located on Chr6p22.2, and likely originate from gene duplication of a common ancestor, Slc17a1/2/3/4, present in teleost fish (131). As SLC17A4 is present only in mammals, other frequently used animal models in thyroid research, such as zebrafish, chicken, and Xenopus, will be of limited use for determining SLC17A4 function in vivo.

Substrate specificity and transport direction SLC17A1-4 were initially classified as $\mathrm{Na}^{+}$dependent inorganic phosphate transporters (129). Three additional related proteins were identified through comparative genomic analyses comprising NPT3 (SLC17A2), NPT4 (SLC17A3), and NPT5 (SLC17A4), which were also designated as inorganic phosphate transporters (130). However, subsequent studies revealed that SLC17A1 and SLC17A3 also accept a broad range of organic anions, which together with the low apparent affinity of SLC17A1 for inorganic phosphate, argued against this classification as inorganic phosphate transporters $(65,130,132,133)$. Indeed, SLC17A4 was also found to transport the inorganic anions aminohippuric acid and urate once expressed in proteoliposomes, the uptake of which could be inhibited by various anionic compounds (65). Whether SLC17A4 also transports these compounds in living cells is unknown.

In a recent genome-wide association study, variation at the SLC17A4 locus was found to be associated with serum free $\mathrm{T} 4$ concentrations (12). Subsequent in vitro studies in a mammalian overexpression system showed that SLC17A4 potently induced the intracellular accumulation of $\mathrm{T} 3$ and T4 (12). Its apparent affinity for T3 ( $\mathrm{IC}_{50}$ : $0.35 \mu \mathrm{M} ; \mathrm{Km} \sim 0.41 \mu \mathrm{M})$ and $\mathrm{T} 4\left(\mathrm{IC}_{50}: 0.06 \mu \mathrm{M}\right.$; $\mathrm{Km} \sim 0.18 \mu \mathrm{M})$ are in the submicromolar range, and are among the highest of all thyroid hormone transporters identified to date (Table 1). Whether SLC17A4 facilitates the efflux of T3 and T4, or the transport of other iodothyronines remains to be studied.

Although these data point to a physiologically relevant thyroid hormone transporter function of SLC17A4, further studies are needed to characterize the functional properties of this transporter, including substrate specificity, efflux potential, transport mechanism, and specific inhibitors. Finally, other members of the human SLC17 family should be functionally characterized to position the SLC17 family among the other transporter families.
Tissue distribution and regulation of expression At the mRNA level, SLC17A4 is expressed in human liver, kidney, colon, small intestine, and pancreas (Fig. 3) $(65,134)$. Likewise, Slc17a4 expression is restricted to the kidney, liver, and gastrointestinal tract in the rat. Immunohistochemistry studies in mice localized SLC17A4 protein to the apical membrane of the small intestinal tract. To our knowledge, no studies have explored the transcriptional regulation of SLC17A4 thus far.

Should SLC17A4 have a similar subcellular distribution in human intestinal cells as observed in rodents, it may be a good candidate to facilitate the uptake of iodothyronines from the gut. Further studies in Slc17a4 ko mice will reveal the relevance of SLC17A4 for cellular thyroid hormone transport in the intestinal tract and will help elucidate the mechanism by which it regulates serum $\mathrm{T} 4$ concentrations.

\section{L-amino acid transporters}

Phylogenesis of L-type amino acid transporters The system L, or L-type amino acid transporters (LATs), comprise a heterogeneous family of proteins that transport neutral (branched chain and aromatic) amino acids in an $\mathrm{Na}^{+}$-independent fashion $(66,83,135)$. The first 2 members, LAT1 and LAT2, are heterodimeric proteins composed of a common heavy chain (CD98; SLC3A2; 4F2hc) and different light chains (SLC7A5 and SLC7A8, respectively). Fifteen additional light chains have been identified and categorized to the SLC7 family, of which 6 others also form heterodimers with CD98 (Fig. 2). However, these members are not classified as LATs because of their differential transporter characteristics, which have been extensively reviewed by Fotiadis et al (2013) (136). Over the years, 3 additional proteins have been identified to exhibit similar transporters characteristics as LAT1 and LAT2 and have been termed LAT3 to $5(71,74,75,137)$. These proteins belong to the SLC43 family (SLC43A1-3) and function as monomeric proteins (reviewed in (138)).

The only members of the LAT family that have been demonstrated to transport iodothyronines in vitro are LAT1 and LAT2. Recent studies showed that LAT3 to 5 do not mediate thyroid hormone uptake, although LAT3 and LAT4 may induce the cellular efflux of 3-mono-iodotyrosine (MIT), 3,5-di-iodotyrosine (DIT), and possibly also 3,3'T2 (67). Two other members of the SLC7 family, $\mathrm{y}^{\star}$ LAT1 (SLC7A7) and $\mathrm{y}^{\star}$ LAT2 (SLC7A6), both closely related to LAT1 and 2 (Fig. 2), were both tested negative for thyroid hormone transport 
(139). Therefore, we will here focus on the characteristics of LAT1 and LAT2.

Substrate specificity and transport direction LAT1 and LAT2 are obligatory exchangers that transport (large) neutral amino acids, including L-leucine, L-isoleucine, L-tyrosine and L-tryptophan, typically in an energy and $\mathrm{Na}^{+}$-independent fashion $(83,140,141)$. LAT2 also accepts the small neutral amino acids glycine and alanine. The apparent affinity for large neutral and aromatic amino acids on the extracellular side is grossly similar for both transporters and falls within the micromolar range, which is close to the physiological concentrations of these substrates in serum, and is up to 100 fold higher than on the cytosolic side (millimolar range) $(66,68,83,140)$. In a variety of cells, including pituitary cells (142), erythrocytes (143), cardiomyocytes (144), astrocytes (145), mouse neuroblastoma cells (146), and thymocytes (147), the uptake of iodothyronines was found to be competitively inhibited by neutral amino acids. These observations suggested the involvement of a LAT or T-type amino acid transporter (148), ultimately resulting in the identification of LAT1 and LAT2 as thyroid hormone transporters. Seminal in vitro studies by Ritchie et al (1999) (149) and Friesema and colleagues (2001) (139) first showed direct thyroid hormone transport by human LAT1 and mouse LAT2, respectively.

LAT1 facilitates the transport of 3,3'-T2, rT3, T3 and T4 into cells, whereas it facilitates the efflux of only 3,3'-T2 (139). Its affinity for T4 (7.9 $\mu \mathrm{M})$, T3 $(0.8 \mu \mathrm{M}), \mathrm{rT} 3(12.5 \mu \mathrm{M})$, and 3,3'-T2 $(7.9 \mu \mathrm{M})$ are considerably lower than for the various amino acids, but still significantly exceeding the physiological thyroid hormone concentrations in serum (Table 1). The inhibition of L-leucine, L-tyrosine, and L-tryptophan uptake (at $10 \mu \mathrm{M}$ ) required supraphysiological concentrations of $\mathrm{T} 3$ ( $\mathrm{K}_{\mathrm{i}} 1.7 \mu \mathrm{M}$ for L-leucine) or $\mathrm{T} 4\left(\mathrm{~K}_{\mathrm{i}} 115 \mu \mathrm{M}\right.$ for L-leucine), whereas transport of T3 (at $0.1 \mu \mathrm{M}$ ) was almost completely blocked in the presence of $100 \mu \mathrm{M}$ L-leucine, L-tyrosine, L-tryptophan, or L-phenylalanine, which is close to the physiological concentrations of these amino acids in human serum $(139,150,151)$.

LAT2 facilitates the uptake of 3,3'-T2 and to a lesser extent T3, but not rT3 or T4, whereas none of the iodothyronine seems to be a suitable substrate for LAT2-mediated efflux $(67,152,153)$. Along this line, the presence of $10 \mu \mathrm{M}$ T3 and T4 had no effect on LAT2-mediated L-alanine, and only minimal effects on L-leucine transport in stably expressing rodent and/or mammalian cell lines
$(150,151)$. The apparent affinity of LAT2 for 3,3'$\mathrm{T} 2$ is $18.6 \mu \mathrm{M}$, whereas its affinity for T3 has not been determined as yet. The uptake of 3,3'-T2 was diminished by supraphysiological concentrations of L-leucine, L-isoleucine, L-methionine, and L-histidine ( $1 \mathrm{mM})$, and by various $\mathrm{T} 1$ and $\mathrm{T} 2$ derivatives, but not by rT3 at a concentration of $10 \mu \mathrm{M}$ (152). Cis-inhibition studies at physiologically relevant concentrations of amino acids have not been reported so far, although similar effects may be expected as observed for LAT1.

LAT1 effectively transports MIT with greater affinity than L-tyrosine, suggesting that the 3-iodination increases substrate affinity (154). Also, the introduction of an a-methyl group in this molecule, resulting in 3-iodo- $\alpha$-methyl-tyrosine (IMT), was well tolerated (154).

The transport of amino acids and iodothyronines by LATs can be competitively inhibited by 2-aminobicyclo(2,2,1)-heptane-2-carboxylic acid $(\mathrm{BCH})$, which is generally considered a LATspecific inhibitor (155). More recently, the LAT1specific inhibitor KYT-0353 (or JPH203) has been synthesized (156). Another inhibitor of LAT2, LAT3, LAT4 but not LAT1 is N-ethylmaleimide (NEM) $(71,157)$. These novel inhibitors may help distinguish the contributions of LAT1 and LAT2 to cellular thyroid hormone transport in future studies. The transport of iodothyronines by LAT1 and LAT2 can also be greatly diminished by cellular amino acid depletion prior to uptake studies, suggesting that LAT1 and LAT2 may require amino acids at the intracellular side to create a suitable gradient for the transport of iodothyronines. This observation contrasts with the inhibitory effect of at least some amino acids on iodothyronine transport once applied at the extracellular side, suggesting that the direction of the amino acid gradient across the cell membrane may importantly determine the rate and direction by which LAT1 and LAT2 transport iodothyronines.

Tissue distribution and regulation of expression In humans and rodents, the CD98 heavy chain has a wide tissue distribution $(68,158)$. Therefore, the tissue distribution of LAT1 and LAT2 is mainly determined by the expression of the SLC7A5 and SLC7A8 light chains, respectively. In rodents, the Lat1 light chain is predominantly expressed in the placenta, brain, vascular endothelial cells, spleen, ovary, testes, retina, and to some extent in renal proximal tubuli at mRNA level (159-161). A similar tissue distribution was observed in humans, with abundant LAT1 mRNA expression in brain, placenta, and testes, as well as in bone, fetal liver, 
and leukocytes (Fig. 3) (68). At the protein level, the presence of LAT1 has been confirmed in microvessels in the mouse brain and spinal cord $(19,162)$. Similarly, LAT1 (and CD98) was detected in bovine (163) and chicken (164) brain capillary cells and in human brain microvasculature endothelial cells (BMECs) (165). Although the localization of LAT1 at the brain capillaries appears consistent across species, it is unlikely to play a major role in the transport of $\mathrm{T} 3$ across the $\mathrm{BBB}$ (see "Mechanisms of Disease- Monocarboxylate Transporter 8 and Organic Anion Transporting Polypeptide-1C1 Deficiency in Human and Animal Models"). LAT1 is also present in primary cultures of mouse neurons and astrocytes, although the contribution of LAT1 to T3 transport in astrocytes appears to be neglectably small (166). Outside the brain, LAT1 protein has been detected in human placenta, where it localized to the apical membrane of trophoblasts with increasing levels toward full-term pregnancy (167).

The expression of Lat 2 mRNA in rodents has been localized to a variety of tissues, including brain, the developing eye, kidney, small intestine, ovary, testes, placenta, and skeletal muscle $(72,83,159,160,168)$. Along this line, LAT2 protein is highly abundant in kidney and small intestine where it localizes to the basolateral membrane of proximal tubuli $(159,160)$, and to the basolateral membrane of small intestinal villi $(160,168)$, respectively. In humans, LAT2 mRNA is very abundantly expressed in the kidney, and to a lesser extent in the placenta, skeletal muscle, liver, spleen, and brain (Fig. 3) (72, 73). In contrast to rodents, human small intestinal cells express only very low levels of LAT2 mRNA (73).

In the developing and mature mouse brain, LAT2 is most prominently expressed in neurons of the cerebral and cerebellar cortex, thalamus, hippocampus, and in choroid plexus structures $(169,170)$. In the developing human brain, LAT2 expression was restricted to microglia cells (169), whereas in the adult human brain, LAT2 protein was also detected in neurons. These observations suggest the presence of a species difference in the regulation of LAT2 expression. Studies of rodent primary cultures indicated the presence of LAT2 in microglia, neurons, and astrocytes. In astrocyte cultures, LAT2 was found to account for $~ 40 \%$ of T3 uptake (166).

The transcriptional regulation of LAT1 and LAT2 expression has not been studied in much detail. Some studies have indicated that LAT1 expression is responsive to estrogen (171) and hypoxia (172), whereas the proto-oncogene c-myc was found to positively regulate $L A T 1$ expression (173). Expression of LAT1 and LAT2 does not appear to be responsive to serum thyroid hormone status (166).

Taken together, LAT1 and LAT2 have been shown to transport a variety of iodothyronines in vitro. However, it is yet unclear whether LAT1 and LAT2 significantly contribute to the cellular uptake of thyroid hormone in vivo. The broad range of alternative substrates as well as the pronounced cisinhibitory effects of physiological concentrations of various amino acids may importantly limit the propensity of LATs to transport iodothyronines in vivo (see also "L-type amino acid transporters").

\section{Monocarboxylate transporter family}

Phylogenesis of the monocarboxylate transporter family The SLC16 gene family comprises 14 members and is also known as the monocarboxylate transporter (MCT) family (Fig. 2) (174). The first members of this family to be identified were MCT1 to 4 , which show the strongest sequence conservation and facilitate the proton-linked transport of monocarboxylate metabolites involved in energy metabolism such as pyruvate, L-lactate, and ketone bodies (174). More recently, MCT7 and MCT11 were found to facilitate the proton-linked transport of ketone bodies and pyruvate, respectively $(175,176)$. Recent studies identified substrates for MCT6 (bumetanide), MCT9 (carnitine), and MCT12 (creatine) $(177,178)$. Genetic mutations in these MCT transporters have been linked to various metabolic disorders (179-181).

The only members of the MCT family found to transport iodothyronines are MCT8 and MCT10 (10, 11). The gene encoding MCT8, SLC16A2, was first identified by Lafrenière et al (182) and is located at the X-chromosome (Chr Xq13.2). The gene was originally called the X-linked PESTcontaining transporter (XPTC) because of the high abundance of Pro (P), Glu (E), Ser (S), and Thr (T) residues in the $\mathrm{N}$-terminal domain of the predicted protein. It consists of 6 exons and 5 introns, of which the first intron is particularly large $(\sim 100 \mathrm{~kb})$. The SLC16A10 gene, encoding MCT10, has a very similar structural organization and is located on Chr 6q21. Both genes are widely conserved across species and are likely to have arisen from a common ancestral gene through gene duplication. MCT8 orthologs have been identified in mammals, rodents, birds, reptiles, amphibians, marsupials, and fish, although only a few of their gene products have been verified as thyroid hormone transporters (10, 183-186). Whereas SLC16A10 has only one 
translational start site (TLS), 2 TLSs have been identified in SLC16A2 of humans and some other mammals such as the cow, elephant, and seal. Most other species, including rat, mice, and zebrafish, have only one TLS corresponding to the most downstream TLS in human SLC16A2. Depending on which of the 2 TLSs is being used, the human MCT8 protein consists of 613 or 539 amino acids, classically referred to as "long" and "short" MCT8, respectively. Because both isoforms exhibit similar transporter characteristics (see the following section) and the homology among species is strongest starting from the second TLS, it is today commonly accepted that short MCT8 is the most relevant isoform. Recently, the MCT8 reference sequence (www.ncbi.org) has been changed from the long to the short isoform, which changes the official amino acid residue numbering. Unfortunately, this renumbering can cause confusion and ambiguity with regard to the nomenclature of variants and mutations in SLC16A2 that have been identified and reported over the last decades, which started counting from the first TLS. To avoid such confusion, we propose continuing the use of the first TLS in the nomenclature of mutations.

\section{Substrate specificity and transport direction}

Early in vitro studies had suggested the involvement of a T-type amino acid transporter in the uptake of thyroid hormones into erythrocytes (187). Such a T-type amino acid transporter was identified and characterized by Kim and colleagues in the early 2000s and termed TAT1 (81, 188). TAT1 belongs to the MCT family (MCT10, SLC16A10) and was found to transport the aromatic amino acids phenylalanine, tryptophan, and tyrosine very effectively, but seemingly not thyroid hormones $(81,188)$. Based on the high sequence homology, MCT8 (SLC16A2) was finally identified as the long-sought T-type amino acid transporter capable of transporting T3 and T4 (10). These studies indicated highly effective transport of T4, T3, rT3, and 3,3'-T2 in oocytes expressing rat MCT8, with apparent $\mathrm{Km}$ values of $4.7 \mu \mathrm{M}$ for T4, $4.0 \mu \mathrm{M}$ for T3, and $2.2 \mu \mathrm{M}$ for rT3 (Table 1). By contrast, overexpression of rat MCT8 did not induce the uptake of the aromatic amino acids or leucine and not of sulfoconjugated iodothyronines or monocarboxylic acids such as lactate and pyruvate (10). Subsequent studies in transiently transfected mammalian cells indicated that (the short isoform of) human MCT8 also induces the intracellular accumulation of T4, T3, rT3, and 3,3'-T2, although the fold induction was less pronounced when compared to rat MCT8 in Xenopus oocytes (76). This apparent discrepancy was later explained by the observation that MCT8 facilitates not only the cellular uptake, but also the cellular efflux of iodothyronines (11). Complementary studies indeed confirmed that upon cotransfection of MCT8 with the cytoplasmic high-affinity thyroid hormone-binding protein mu-crystallin, the intracellular accumulation of T3 and T4 was strongly amplified by preventing MCT8-mediated thyroid hormone efflux (11). Expression of MCT8 greatly enhances the intracellular metabolism of iodothyronines, in particular by $\mathrm{DIO} 3$ (76, 189), which provided ultimate proof that MCT8 regulates the amount of intracellular thyroid hormone levels. Using a similar approach, later studies demonstrated that MCT10 is an equally proficient T3 transporter as MCT8, but substantially less effective with T4 as a substrate (11). Based on the abovementioned studies, the transport of thyroid hormones by MCT8 and MCT10 likely concerns facilitated diffusion and is not sensitive to $\mathrm{pH}$ or dependent on $\mathrm{Na}^{+}$. Detailed (cis-inhibition) studies on potential substrates and inhibitors for MCT8 suggested that MCT8 is specific for the L-enantiomers of thyroid hormones, and requires both the amino and the carboxy groups of the alanine side-chain of thyroid hormone as well as at least one iodine atom in each iodothyronine ring (77), although 3-iodothyronamine at high concentrations reduced MCT8-mediated thyroid hormone uptake $(77,190)$. In contrast to MCT8, T3 uptake by MCT10 was also effectively inhibited by $1 \mu \mathrm{M}$ 3-T1, 3',-T1, and 3,5-T2, suggesting that the presence of iodine in both rings might be a less stringent requirement for MCT10 (78). Moreover, rat MCT10-mediated uptake of tryptophan is inhibited by L-3,4-dihydroxyphenylalanine (L-dopa) and 3-O-methyl-dopa (188). Direct uptake studies confirmed that compounds lacking the aNH2 group of thyroid hormone (ie, Triac and tetraiodothyroacetic acid [Tetrac]) are not suitable substrates for MCT8 and MCT10, whereas its modification (ie, N-bromoacetyl-iodothyronines) is tolerated (191-193). Similar studies confirmed that MCT10 directly transports 3,5-T2 and L-dopa (78, $81,188)$. No substrates other than iodothyronines have been identified for MCT8 thus far. MCT8mediated thyroid hormone uptake is inhibited by the nonselective inhibitor BSP (77). The tricyclic antidepressant desipramine inhibits both MCT8 and MCT10 (194), whereas the flavonolignan silychristin appears to be a specific inhibitor of MCT8 (79). Moreover, several tyrosine kinase inhibitors have been found to interfere with MCT8 and MCT10 function through noncompetitive 
inhibition (195-198). Although not as extensively studied as short MCT8, the long isoform of human MCT8 also efficiently transports iodothyronines $(80,199)$.

Expression and tissue distribution of monocarboxylate transporter 8 and monocarboxylate transporter $10 \mathrm{MCT} 8$ is expressed in many human tissues (Table 1 and Fig. 3). MCT8 mRNA levels are highest in the liver and adrenal gland and somewhat lower in a variety of other tissues including the brain, kidney, placenta, and thyroid (44, 82, 108, 200-202). Detailed studies by Alkemade et al showed that MCT8 is present in neurons and astrocytes of the paraventricular and infundibular nuclei in human hypothalamus (203). Expression of MCT8 was also detected in human tanycytes, a specialized ependymal cell type lining the third ventricle and involved in the negative-feedback regulation within the hypothalamus-pituitary-thyroid (HPT) axis $(203,204)$. Analysis of human fetal cerebral cortex at midgestation revealed MCT8 immunopositive signals in numerous neurons of the ventricular and subventricular zone, in choroid plexus structures and ependymal cells lining the ventricle, as well as in the wall of microvessels $(108,169,205)$. The presence of MCT8 protein in these cells was further detailed by extensive spatiotemporal expression analyses in human brain tissues from GW14 to GW38 (58). At all stages, strong immunoreactivity was observed within vascular structures in all brain regions and at GW32 and GW38 in their surrounding astrocytes as well, consistent with the current belief that MCT8 is important for the transport of thyroid hormones across the BBB (see "Role of MCT8 at Brain Barriers"). In line with previous studies (82), MCT8 was also present in choroid plexus epithelial cells (apical > basolateral membrane) and fenestrated capillaries throughout development. From GW20 onward, MCT8 was increasingly detected in the apical membrane of ependymocytes, in particular those facing the fourth ventricle, and to a lesser extent in the cilia and basal processes of these cells. In addition, MCT8 exhibited strong immunoreactivity in the leptomeningeal cells and blood vessels in the subarachnoid space at all ages. Thus, MCT8 appears to also be expressed at the inner and outer CSFBB. MCT8 protein was also detected along the entire length of radial glial cells, cortical plate neurons, and Cajal-Retzius cells. Immature neurons in the cortical plate and subplate show only weak perinuclear MCT8 staining between GW16 and GW25, whereas strong membrane staining starts to become apparent by GW32.
In the adult human CNS, MCT8 immunolabeling was also present in microvessels and choroid plexus structures, whereas neuronal MCT8 expression appeared to be weak $(108,169)$.

In rodents, the MCT8 protein has been detected in the sinusoidal membrane of hepatocytes (10, 20 ), on the basolateral membrane of thyrocytes $(19,206,207)$ and the proximal tubule cells in the kidney $(208,209)$, retinal cells (210), placenta (211), and in different cell types in skeletal muscle (24). In mouse brain, MCT8 is predominantly localized in different neuronal populations of the cerebral and cerebellar cortex, hippocampus, striatum, and hypothalamus, with higher expression during early postnatal stages. MCT8 mRNA was also detected in oligodendrocytes and in astrocytes $(106,108,169,212-214)$. Similar to the human situation, mouse MCT8 protein is strongly expressed in capillary endothelial cells, choroid plexus structures and in tanycytes $(169,204,212)$. In zebrafish, mct 8 mRNA is expressed in different areas of the brain, spinal cord and vascular system $(29,185,215)$. Colocalization studies indicated that mct 8 mRNA is expressed in sensory and motor neurons, oligodendrocytes, but not astrocytes (29, $30,216)$, which is reminiscent to the situation in mice. Mct8 is, among others, also expressed in brains and brain barriers of Xenopus and chicken $(32,164,184,217)$.

Taken together, extensive expression studies have indicated that MCT8 is highly abundant in all brain barriers, most important the BBB, and in many thyroid hormone target cells within the brain. In all species, the cell-type specific expression pattern of MCT8 was found to vary depending on the brain region studied and the timing during development (170, 218), which should be considered when comparing different models. The identification and characterization of MCT8-expressing cell types will help further delineate the role of MCT8 in brain thyroid hormone homeostasis (see "Mechanisms of DiseaseMonocarboxylate Transporter 8 and Organic Anion Transporting Polypeptide-1C1 Deficiency in Human and Animal Models"). In addition to animal models, redifferentiated human iPSCs (28), or human embryonic stem cells (219) may provide complementary models to study the role of MCT8 in these cells. Further studies should also disentangle whether the long isoform of the human MCT8 protein is also expressed in vivo, as currently available studies presumably detected the short isoform of MCT8 (169) or were not suited to differentiate between both isoforms. Establishment of an antibody that specifically recognizes the extended $\mathrm{N}$-terminal tail may help to resolve this intriguing question. 
In humans, MCT10 mRNA expression was predominantly detected in the kidney, skeletal muscle, placenta, heart, and developing brain (Fig. 3) (44, 81, 202, 220). MCT10 mRNA expression increased with gestational age in the human placenta, and its expression levels were lower in fetuses presenting with intrauterine growth restriction (202). MCT10 protein has been detected at various stages and regions in the developing brain (220) and on the apical membrane of the choroid plexus (82). In the developing human hypothalamus, MCT10 expression was detected in different neuronal cell populations, including the paraventricular nucleus, supraoptic nucleus, lateral hypothalamus, and infundibular nucleus starting from the second trimester, although the expression levels and distribution pattern showed interindividual variation and changed during development (82). In rodents, MCT10 is expressed in a great variety of tissues, including the thyroid, liver, kidney, chondrocytes, small intestine, and skeletal muscle $(19,24,221,222)$. Of note, Mct10 RNA expression in the murine brain is low and appears to be restricted to a subset of neurons, microglia cells, and oligodendrocytes $(166,170)$, whereas Mct10 RNA is also highly enriched in mouse choroid plexus at embryonic stages (E15) (223). The tissue distribution of $m c t 10$ in zebrafish has not been studied in much detail and appears to be restricted to the liver and trigeminal ganglia (29). The differences in expression of MCT8 vs MCT10 suggest that absence of each of the individual transporters is unlikely to be fully compensated by the other.

Little is known about the molecular mechanisms involved in the transcriptional regulation of MCT8 and MCT10. Kogai et al identified an enhancer element responsive to retinoic acid that enhanced expression of MCT8 in F9 mouse teratocarcinoma cells (224). However, this element is not present at the corresponding location in the human genome and, thus, it is currently unclear whether the expression of the human MCT8 gene is regulated by retinoic acid. Inspection of the MCT8 proximal promoter sequence in different species suggests that MCT8 lacks a classical TATA-box element and is thus regulated through other mechanisms $(29,224)$. Although other specific factors and pathways that regulate MCT8 (and MCT10) expression, especially in physiological relevant tissues, remain to be identified, several studies may provide some guidance for identifying such mechanisms. Recent studies in rodents indicated that expression both of Mct8 and Mct10 is responsive to feeding status and systemic inflammation because pronounced downregulation of hepatic and hypothalamic Mct8 expression levels was found during fasting (225-227) and reduced Mct8 expression in liver and brain vasculature during systemic inflammation $(111,225)$.

It still remains to be clarified to what extent MCT8 expression is regulated by thyroid hormone status. Whereas no significant changes in brain Mct8 transcript levels were reported in congenital hypothyroid Pax8 ko mice (212), Mct8 expression was downregulated in tanycytes of hypothyroid rats (228). Conversely, testicular Mct8 expression was increased in hyperthyroid rats (229). In developing zebrafish, global mct8 expression levels were reported to be thyroid hormone responsive (110). Therefore, MCT8 expression might be regulated by thyroid status in a tissue-specific fashion. Yet, the underlying molecular mechanism and the involved thyroid hormone-responsive factors remain to be identified. Identification of such factors would help to disentangle why the expression of MCT8 and OATP1C1 appear to respond differently to thyroid state. Other studies may further support the link between the regulation of MCT8 expression and thyroid function. First, MCT8 expression levels were increased in thyroid tissue from patients with Graves disease and in the follicular thyroid cell line PCCL3 after stimulation with thyrotropin (TSH), suggesting that MCT8 expression in the thyroid may be TSH responsive (230). Second, both thyroidal Mct8 and Mct10 mRNA expression levels were downregulated in thyrocyte-specific TR $\beta$ deficient mice (231). Finally, Mct8 expression in the thyroid gland appears to respond to iodine status in rodents (232-235). These and other studies (eg, (205, 214, 236-239)) may pave the way for future studies on the transcriptional regulation of MCT8 and MCT10.

With their Km values for T3 (and T4) uptake well above the serum free hormone concentrations in vivo (see "Substrate Specificity and Transport Direction"), it is unknown to what extent expression levels should change to achieve relevant effects on the cellular transport of thyroid hormone. Importantly, the $\mathrm{Km}$ of thyroid hormone efflux by MCT8 and MCT10 is unknown as well as the intracellular thyroid hormone concentrations in proximity of the transporter. Therefore, it is not excluded that the level of expression is of differential importance for the uptake and efflux rate.

\section{Molecular Mechanism of Thyroid Hormone Transport}

\section{Structure and transport mechanism of trans- membrane transporters}

Most plasma membrane transporter proteins in humans belong to either the 
adenosine triphosphate-binding cassette (ABC) or to the SLC gene family. All currently known thyroid hormone transporters belong to the SLC family. The known thyroid hormone transporters have different polypeptide chain fold patterns, including the major facilitator superfamily (MFS) fold (MCTs and OATPs), the LeuT fold (LAT1 and LAT2), and the NhaA fold (NTCP) (Fig. 4A-4C). The MFS fold contains 2 nearly symmetrical bundles of 6 transmembrane domains (TMDs) that can exert a rotary movement against each other. The presence of the appropriate substrate induces a conformational change from the outside-open to the inside-open conformation (uptake) and vice versa (efflux) (240). The LeuT fold contains 2 inverted bundles of 5 TMDs followed by 2 TMDs that do not participate in this pseudosymmetry. TMD1 and TMD6 are discontinuous and interrupted by a highly conserved nonalpha-helical segment involved in substrate binding (241).
The NhaA fold comprises 2 inverted segments of 5 TMDs with TMD4 and 9 being unwound in the center to allow substrate interactions. For more details, please refer to a recent review by Schweizer et al. (242). The structure-function relationship of the known thyroid hormone transporters is best studied in MCT8 and LAT2.

\section{Structure function of monocarboxylate trans- porter $\mathbf{8}$ and monocarboxylate transporter $\mathbf{1 0}$}

The MCT family is classified as a member of the MFS and shares a common topology with 12 TMDs and intracellular C- and N-termini (243) (Fig. 4A). No crystal structures are available for MCT8 or MCT10, or for any of the other MCTs. Therefore, structural information is mainly derived from analogies with (closely) related proteins through protein homology modeling and supported by in vitro studies that have evaluated the effects of amino acid substitutions and chemical modifiers on transport function. (a)

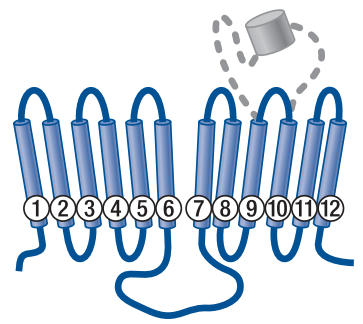

(b)
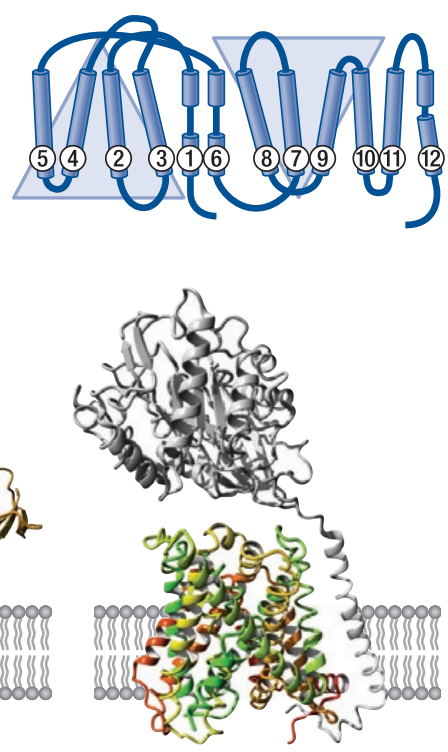

LAT1 and CD98 (c)
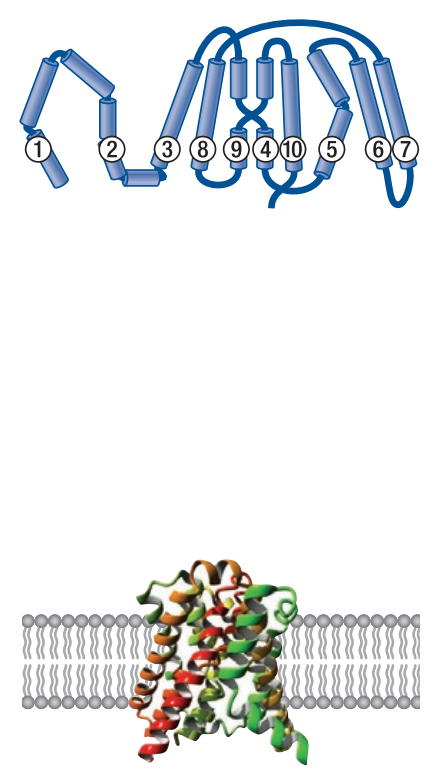

ASBTyf

Figure 4. Schematic overview of the different protein folds of thyroid hormone transporter families. A, The MCT and OATP families both belong to the major facilitator superfamily (MFS), which is organized in 2 symmetrical bundles of 6 transmembrane domains (TMDs) linked through a large intracellular loop. MFS proteins likely transport substrates according to the "rocker switch model" in which the presence of substrate induces a conformational change that leads to the uptake or efflux of the substrate molecule. Members of the OATP family contain a large extracellular loop between TMD9 and TMD10 with a KAZAL-like sequence motif (in gray). Displayed are exemplary homology models of MCT8 (based on the E coli fucose:proton symporter FucP, PDB\#3O7P) (245,246) and OATP1C1 (based on the $E$ coli multidrug transporter MdfA PDB\#4ZP0 and the KAZAL-type inhibitor Infestin 4 (PDB\#2ERW) (16,288,289). B, LAT1 (and LAT2) are organized according to a LeuT fold, which contains 2 inverted bundles of 5 TMDs followed by 2 TMDs that do not participate in this pseudosymmetry. Displayed is the recently published crystal structure of LAT1 in complex with CD98 (PDB\# 6IRT) (260). C, The NTCP protein structure is organized according to the NhaA fold, which comprises 2 inverted segments of 5 TMDs, with TMD4 and 9 being unwound in the center to allow substrate interactions. Because no homology models of NTCP have been reported in the context of thyroid hormone transport, its putative structure is illustrated by the structurally related bacterial homolog of the bile acid sodium symporter ASBTyf protein (PDB\# 3ZUX), which may serve as a template for homology modeling (290). 
The first 3-dimensional MCT8 protein homology model was published by Kinne and colleagues (2010) and was based on the crystal structure of the bacterial glycerol-3-phosphate transporter GlpT in the inward-open conformation (PDB ID: 1pw4) (77, 244). The resulting model predicted a critical role for positively charged Arg445 (TMD8) and negatively charged Asp498 (TMD10) in T3 transport because of their location within the membrane plane. This notion was supported by in vitro studies that showed the deleterious effects of their respective alanine substitutions (77). The model also predicted a salt bridge between both residues (77). This prediction is experimentally supported by studies of a charge reversal mutant (Arg445Asp/Asp498Arg) that regained activity compared to both single mutants (245). In analogy to the binding mode of $\mathrm{T} 3$ to the $\mathrm{T} 3$ receptors and the catalytic side of DIO3 $(246,247)$, it was postulated that MCT8 contains a so-called His-Arg clamp (248). Three of such His-Arg pairs were identified in the inwardopen model of MCT8, comprising residue pairs His192/Arg445, His192/Arg301, and His415/ Arg301 (77, 248). The existence of the His192/ Arg445 clamp is best supported by in vitro data. Substitution of His 192 by Phe or Ala affected transporter kinetics (249) and His192 was identified as a target for the His-reactive chemical reagent diethylpyrocarbonate (DEPC) (250). The modification by DEPC was fully prevented in presence of substrate, suggesting that the imine side chain of His 192 aligns the substrate pore (250). Although not identified as a target for DEPC modification (250), substitution of His415 by Phe or Ala reduced thyroid hormone uptake function and altered transport kinetics (249). In contrast, limited experimental evidence is available supporting a role of Arg301 in substrate binding. In fact, substitution of this residue by Ala greatly impaired protein stability, rather suggesting a role for this residue in the maintenance of protein stability (249). Additional structure-function information has been derived from studies using the membraneimpermeable cysteine-specific modifying reagents p-chloromercuribenzenesulfonate and mercury(II) chloride, which identified Cys481 and Cys497 as accessible target residues (251).

For a long time, the generation of homology models in transporter conformations other than the inward-open conformation had been limited by the paucity of available crystal structure templates with sufficient homology to MCT8 (242). By combining several template crystal structures, Protze et al (2017) recently established 3 MCT8 homology models in different conformations allowing further delineation of putative mechanisms by which substrate molecules transit the substrate pore (252). These studies further confirmed that Arg445, Asp498, and His192 are located at the center of the substrate pore and are key players in the docking of substrate in the inward-open and outward-open conformation. In addition, these studies suggested an important role for several other residues in maintaining protein stability (Lys418, Tyr419, Ser313, Ser314, Tyr503) or substrate recruitment (Glu422, Glu423). In parallel, an alternative homology model in the outward-open conformation based on the $E$ coli fucose:proton symporter suggested that the interaction between Arg445 and Asp498 is modulated in the presence of substrate $(253,254)$. In vitro chemical modification studies have supported the involvement of Arg445 in substrate docking by identifying Arg445 as an accessible target for phenylglyoxal modification, which was prevented in the presence of substrate (253). Model-based mutagenesis studies furthermore pointed to a putative role for Phe189, Phe279, and Phe287 in substrate docking through the formation of pi-pi interactions with the phenolic rings of iodothyronines (253). Of note, mutation of Tyr184 (the corresponding residue of Phe287 in MCT10) to a Phe allows T4 transport by MCT10 (78), supporting the involvement of this residue in substrate docking and selection. Additional information has been derived from the in vitro characterization of mutations identified in patients with MCT8 deficiency (see "Genetic Mutations in SLC16A2"). The impact of these mutations on MCT8 function in different testing systems has been recently extensively summarized by Braun and Schweizer (255).

Despite its $49 \%$ amino acid sequence identity and $\sim 80 \%$ sequence similarity to MCT8, predominantly concerning the 12 TMDs, MCT10 lacks sufficient homology to the available crystal structure templates. This poses a limitation for the generation of structural homology models of MCT10 and therefore progress of structure-function studies is hampered. In addition to the above mentioned study by Johannes et al (2016), a recent mutational screen in MCT10, using the yeast Saccharomyces cerevisiae, suggested the involvement of Asn 81 in the recognition of aromatic amino acid substrates (256). The impact of this and the other variants evaluated in this study on T3 transport was not tested.

The exact function of the extended $\mathrm{N}$-terminal domain of MCT8, which is unique to the long isoform, has not been fully elucidated. Recent 
studies have suggested that the extension of the N-terminal Pro- (P-), Glu- (E-), Ser- (S-), Thr- (T-) rich region, known as the PEST domain (257), makes the MCT8 protein more susceptible to degradation through the ubiquitin-proteasome system (199). So far, there is no theoretical or experimental evidence that any of the MCT family members is being glycosylated (243). Some MCT proteins require ancillary proteins that govern proper plasma membrane translocation (258). It is currently unclear whether the 2 thyroid hormone-transporting members, MCT8 and MCT10, require such ancillary proteins; if so, such factors differ from those required by MCT1 to 4 (259). Different experimental approaches have demonstrated the presence of MCT8 homodimers and multimers in cell lysates and living cells (259-261). The physiological relevance of these complexes and the domains involved in establishing these interactions are currently unknown. MCT8 was found to interact with pituitary tumor-transforming gene-binding factor, which impeded cell membrane expression of MCT8 in tumor cells overexpressing this factor (262). Recently, MCT8 was also found to interact with the TSH receptor, which is a G-coupled membrane receptor, and modulate its activity (263). The relevance of these interactions in physiology remains to be explored. No other proteins that form direct interactions with either MCT8 or MCT10 have been identified thus far.

\section{Structure function of L-type amino acid transporters}

LAT1 and LAT2 belong to the superfamily of the amino acid/polyamine/organocation transporter (APC), and are classified as heteromeric amino acid transporters (HATs) (264). Both proteins require heterodimerization of the LAT1 (SLC7A5) and LAT2 (SLC7A8) light chains with the common heavy chain CD98 (SLC3A2) (265). LAT1 and LAT2 are nonglycosylated proteins that contain 12 TMDs organized in a LeuT fold pattern (136) (Fig. 4). CD98 is a type II membrane N-glycoprotein that contains $4 \mathrm{~N}$-linked glycosylation sites, a single TMD, an intracellular N-terminus, and a large extracellular C-terminus (136). Crystallization of the LAT1 and LAT2 CD98 complexes has long been unsuccessful. Although the atomic structure of the extracellular domain of CD98 was resolved in 2007 (266), until recently structural information on the CD98/LAT1 and LAT2 heterodimers was mainly derived from transmission electron microscopy, single-particle analysis, chemical crosslinking studies, mutational screens, and structural modeling (265, 267). However, Yan and colleagues recently resolved the structures of the LAT1 and CD98 complex using cryoelectron microscopy (Fig. 4B) (268), which will greatly advance the understanding on LAT1 and LAT2 function. This review will highlight several key aspects on the structure function of LATs derived from studies prior to this recent landmark study.

Compiling evidence has suggested that the light chains of LAT1 and LAT2 comprise the functional transporter, whereas CD98 functions as a chaperone protein that targets the complex to the cell membrane and increase protein stability (265). Heterodimerization of the light and heavy chain is established through a disulfide bond between a conserved Cys residue located in the second extracellular loop of LAT and Cys109 located just outside the single TMD at the extracellular site of CD98 (66), but probably also involves other domains of both proteins (268). Structural homology models of LAT1 (269-273) and LAT2 (265) have been generated based on the crystal structure of the L-arginine/Agmatine Antiporter from $E$ coli (AdiC; PDB: 3L1L, (241), or PDB: 3OB6, (274)) or the APC transporter Apct from Methanocovvus jannaschii (PDB: 3GI8, (275)), both members of the APC superfamily. These models have been used to identify critical residues involved in substrate recognition and translocation $(269,272)$ as well as in the design of novel LAT1-specific inhibitors (273).

In the context of iodothyronine transport, LAT2 has been modeled on the structures of AdiC and Apct using a chimeric modeling approach (276). The resulting model has been used to guide targeted mutational studies to identify residues that determine substrate specificity, which have been recently reviewed by Krause and Hinz (277). Side-chain shortening of Tyr130 to an Ala or Asn 51, Asn133, and Asn248 to a Ser increased 3,3'-T2 transport by enlarging the predicted substrate traversing channel, whereas side-chain enlargement of Thr140 to a Phe, or Tyr130 to an Arg diminished 3,3'-T2 transport by occluding the traversing channel (276). In contrast, shortening of the Tyr130 residue to an Ala, thereby enlarging the traversing channel, increased the uptake of T3 and allowed some uptake of T4 by LAT2, which is normally limited because of steric hindrance (153). Some of these substituents did not affect the transport of L-leucine or L-phenylalanine (153), suggesting that these residues play a role in the establishment of a structural niche that accommodates the bulky second aromatic ring and/or bulky iodo-substituents, or function as a steric gate keeper for rigid and bulky molecules. Moreover, these studies underlined the importance 
of an aromatic residue at position 242 (Phe/ Trp242) to allow uptake of 3,3'-T2, and suggested a role of Tyr130, Asn133, and Phe242 in determination of transport directionality (153). By varying different moieties of the substrate molecule, it has been shown that the presence both of the carboxyl and amino group is required to render a molecule a substrate (or inhibitor) for LAT2, and that the presence of iodine substituents, especially at the tyrosyl-ring, increases substrate suitability as long as these moieties do not restrict the flexibility of the phenolic ring $(153,276,277)$. Based on their different substrate specificity and preference, such substrate (and inhibitor) requirements likely differ between LAT1 and LAT2 (270-273). The interpretation of these findings remains to be verified using models based on the novel LAT1 structure (268).

\section{Structure function relationship of organic anion transporting polypeptides}

The OATPs belong to the MFS family, and their 12 transmembrane helices are presumably organized according to the MFS fold (Fig. 4A) $(84,278,279)$. None of the structures of the OATPs have been resolved yet. Characteristic of the OATP family is the so-called KAZAL-motif in extracellular loop connecting TMD9 and TMD10. Although the function of this region is not clear, studies on OATP2B1 indicated that the highly conserved Cys-residues within this region form disulfide bonds required for proper surface expression (280). Moreover, this loop yields (a) target site(s) for N-glycosylation involved in the control of membrane expression of OATP $(280,281)$. The $\mathrm{pH}$ sensitivity of OATPs was found to be mediated through a strongly conserved His residue in the third TMD, which is absent in the $\mathrm{pH}$-insensitive OATP1C1. Replacing the Glu found at this conserved position by a His residue also rendered OATP1C1 pH sensitive (55). Based on these observations, OATPs likely share some basic structural characteristics in regions outside the substrate channel. As a corollary, the composition of the substrate channel likely differs among OATPs.

Most OATPs accept multiple substrates and therefore require (a) flexible substrate binding site(s) to accommodate a variety of chemical structures. Several groups have strived to identify the substrate-interacting residues and delineate the transport translocation mechanism of OATPs. However, most of these studies focused on transporters other than OATP1C1 or substrates other than iodothyronines (282-294). Therefore, it is unclear to what extent these findings also apply to the transport of iodothyronines.
Only a few studies have focused on the structure function of OATP1C1 in relation to T4.

Because the affinity of OATP1C1 toward T4 is 2 orders of magnitude higher than that of other OATPs (91), it is very likely that the substrate channel of OATP1C1 has been selectively adapted to facilitate T4 transport. The presence of an atypical kinetic profile moreover suggested that OATP1C1 yields more than one substrate binding site for T4 (97). By combining structural modeling of rat OATP1C1 to the lactose permease, multidrug transporter EmrD, and GlpT and targeted mutagenesis studies, the highly conserved Arg601 residue within TMD11 has been implied in attaining substrate selectivity and in potentiating T4 affinity at its high-affinity binding site (295). However, the low sequence similarity between OATP1C1 and the templates available at that time, as well as the lack of several domains in these models, may prevent accurate structure function studies. Recently, a chimeric structural model of human OATP1C1 to the more homologous $E$ coli multidrug transporter Mdfa has been reported, using a KAZALtype inhibitor Infestin 4 to model the large, and functionally relevant, extracellular loop between TMD9 and TMD10 $(16,296,297)$. This model may help to further delineate the substrate translocation mechanism of OATP1C1 and to elucidate how OATP1C1 achieves its remarkably high affinity for T4 and rT3, but not T3.

\section{Structure-function relation of other thyroid hor- mone transporters}

Similar to its bacterial homolog bile acid sodium symporter ASBT $_{\gamma f}$ (298), NTCP likely has a NhaA polypeptide fold and consists of 10 TMDs with an intracellular $\mathrm{N}$-terminus and C-terminus (Fig. 4C). Over recent decades, several structural models of NTCP have been generated, mainly based on $\mathrm{ASBT}_{\mathrm{\gamma f}}$, and several amino acid residues have been identified as important determinants of bile acid transport. These studies have been extensively reviewed elsewhere and focused mostly on bile acid transport. Therefore, structural requirements for the transport of (sulfoconjugated) iodothyronines have not been established yet.

Structural models and structure-function studies of SLC17A4 are currently lacking. Like other members of the SLC17 family, SLC17A4 presumably follows the MFS polypeptide fold (128). The establishment of a structural model of SLC17A4 and a more detailed functional characterization will help elucidate how this transporter interacts and translocates its substrates. 


\section{Common features of substrate recognition}

Because the various thyroid hormone transporter (families) have different structural folds and have different need for cosubstrates or countersubstrates or ions, delineation of a common substrate recognition and translocation mechanism is a laborious task. From the available data it can be deduced that spatial restraints of the substrate binding pocket importantly determine substrate specificity. Even slight elongation of amino acid side chains may compromise substrate transport or alter substrate selectivity in LATs $(153,276,277)$, MCTs $(77,78$, $252,253)$ and probably also the other transporter families. Yet, little is known about the required interactions between substrate and transporter to induce conformational changes on substrate binding. At least MCT8 and OATP1C1 likely require charged residues along the substrate channel to balance the charged groups of the alanine side chain of thyroid hormone molecules. The presence of an His-Arg clamp in MCT8 as well as the thyroid hormone receptors and DIO3 may suggest that different thyroid hormone interacting proteins share common substrate recognition mechanisms. It is yet unclear whether other thyroid hormone transporters share this signature. Identification of a common structural signature for thyroid hormone binding would offer great opportunities such as screening for novel thyroid hormone transporters and designing target-specific drugs. Resolving the structure of additional thyroid hormone transporters will help to further disentangle the substrate translocation mechanism of the various transporters and allow more detailed comparisons between the transporters and other thyroid hormone-binding proteins.

\section{Clinical Relevance of Thyroid Hormone Transporters}

\section{The impact of single-nucleotide polymorphisms on thyroid hormone transporters}

Background on common genetic variation in thyroid hormone transporter genes Whereas a subset of rare genetic variants (mutations) in thyroid hormone transporter can cause apparent clinical disorders, the effects of other, more common genetic variants in thyroid hormone transporter genes may have more subtle effects on thyroid hormone regulation. To delineate the relevance of such genetic variants, several studies have investigated the relation between single-nucleotide polymorphisms (SNPs) in thyroid hormone transporter genes and serum thyroid hormone concentrations. Some of these studies also evaluated the impact of SNPs on thyroid hormone transporter function in vitro. In addition to these candidate gene approach polymorphism studies, several genome-wide association studies sought to identify genetic factors that determine serum FT4 and TSH concentrations in healthy individuals and patients with thyroid disease (eg, $(12,299))$. The effectiveness of this approach is illustrated by the recent identification of SLC17A4 as a thyroid hormone transporter (12).

Most studies on the association between common variants in thyroid hormone transporter genes and serum thyroid hormone concentrations have been extensively reviewed by van der Deure et al (42). Together these studies have demonstrated that careful evaluation of common variants in thyroid hormone transporter genes can provide new insights into the physiological role and activity of thyroid hormone transporters. However, serum thyroid hormone concentrations do not necessarily reflect tissue thyroid hormone status. Therefore, the absence of significant associations between genetic variants and serum thyroid hormone concentrations does not exclude a physiological function for the involved gene(s) in the regulation of thyroid hormone action at the level of individual tissues or cell populations.

Genetic variation in monocarboxylate transporter 8 and monocarboxylate transporter $10 \mathrm{To}$ date, 3 studies have been reported on the relationship between the 2 established MCT8 polymorphic variants and serum thyroid hormone concentrations (300-302). The first variant (ChrX:73641569 T/C; rs6647476) results in a p.Ser107Pro substitution in the intracellular N-terminal part of MCT8. LagoLestón and colleagues genotyped 276 healthy Spanish men for this polymorphism and found no association with the serum thyroid hormone concentrations or with mRNA levels coding for MCT8 or thyroid hormone-responsive genes in white blood cells or in T3-stimulated fibroblasts (301). Likewise, van der Deure et al found no association between this polymorphism and serum thyroid hormone concentrations in a cohort of 156 healthy men and women (300). A more recent study by Roef et al, who looked at this and other variants in the Siblos ( $\mathrm{n}=893 \mathrm{men}$ ) and Asklepios ( $\mathrm{n}=1164$ men and $\mathrm{n}=1166$ women) cohorts, did not find any association between the p.Ser107Pro variant and serum TSH or FT4 concentrations. In this study, serum FT3 concentrations were found to be lower in male but not female carriers. The significance of this finding as well as the impact 
of the p.Ser107Pro mutations on MCT8 function remain to be elucidated. In 2 independent studies, male carriers of a different polymorphism located in intron 5 (ChrX:73749319 T/G, rs5937843) had lower FT4 concentrations than wild-type male participants, but this was not observed in homozygous female carriers $(300,302)$. Should such findings be replicated in larger cohorts, further studies are warranted to unravel the mechanism underlying the (sex-specific) effects of this SNP.

Medici et al found a borderline significant association between the rs17606253 variant of the MCT10 gene (Chr6:111205242 T/C) with serum FT4 concentrations (303). This variant is located within the third intron of MCT10, a region of high linkage disequilibrium that also includes exons 4 to 6 coding for part of the TMD and C-terminus. The exact variant underlying the association is thus yet unclear. A more recent study suggested that individuals harboring this variant in combination with 1 of the 3 variants studied in DIO2 (rs225014, rs22501, or rs12885300) may modulate hypothyroid patients' preference for levothyroxine (L-T4) + liothyronine (L-T3) replacement therapy over L-T4 alone (304). Further studies should reveal whether carriers of these variants also exhibit biochemical differences in response to both therapeutic strategies. Another common polymorphism (Chr6:111222741 C/A, rs14399) in the 3'-UTR of the MCT10 gene has been repeatedly shown to have no impact on serum thyroid function test (300, 302).

Genetic variation in organic anion transporting polypeptides Most OATPs, except for OATP1C1, have a low specificity toward thyroid hormone and have been implied in the transport of many xenobiotics. For this reason the impact of genetic variants in OATPs has been most widely studied in the context of drug pharmacokinetics. These studies found significant associations between multiple variants in OATPs and the pharmacodynamics of various compounds (eg, (45,305-308)). Importantly, none of the common genetic variants in any of the thyroid hormone-transporting OATP encoding genes showed a statistically significant association with serum FT4 or TSH concentrations in the meta-analysis by Medici et al, which were in line with other studies in smaller cohorts $(42,302)$. Because OATPs also transport iodothyronines other than T4, these studies also investigated associations with serum T3, rT3, and T4S concentrations.

The Chr12:21487544 A/G (rs10841795; p.Ile13Thr) common variant in OATP1A2 was associated with higher serum T3 concentrations in healthy blood bank donors (42). Except for transport of T4S, this variant showed no differences from wild-type (WT) in thyroid hormone transport function in vitro. Despite the absence of consistent associations with serum thyroid hormone concentrations, another common variant in OATP1A2 (Chr12:21457434 T/G, p.Glu172Asp, rs11568563) reduced the uptake and subsequent intracellular metabolism of (sulfoconjugated) T3 and T4 in vitro (42).

The 12:21331549 T/C (rs4149056) that results in a p.Val174Ala substitution in OATP1B1 was associated with higher serum T4S concentrations in healthy blood donors (49). Subsequent in vitro studies demonstrated that this variant diminished the cellular uptake of T4S in transfected mammalian cell lines. Together, these findings may indicate a role for OATP1B1 in the uptake of T4S and possibly also other sulfoconjugated iodothyronines in the liver. None of the studied common variants in the highlyhomologous OATP1B3 (Chr12:21011480 T/G, p.Ser112Ala, rs4149117; and Chr12:21015760 G/A, p. Met233Ile, rs7311358, which are in complete linkage disequilibrium with each other) were found to be associated with serum thyroid hormone concentrations, including rT3 and T4S (42).

Common variants in the OATP1C1 gene (12:20864342 C/A, p.Pro143Thr, rs36010656; Chr12:20702827 C/T, intron 3, rs10770704; Chr12:20753056 C/T, c.C3035T 3'-UTR, rs10444412) were not consistently associated with serum thyroid hormone concentrations $(57,302)$. The p.Pro143Thr and c.C3035T 3'-UTR variants did not affect OATP1C1-mediated uptake of T4 or rT3 and did not diminish the metabolism of these compounds in vitro (57). Despite the absence of significant associations with serum thyroid hormone concentrations, the intron $3 \mathrm{C} / \mathrm{T}$ and c.C3035T 3'-UTR variants were associated with fatigue and depression in a cohort of hypothyroid patients (309). The mechanism behind this observation is still unclear. Recently, homozygosity for the minor allele of the Chr12:20710639 A/G variant (rs1515777) has been associated with a decrease in serum FT3 and FT3:FT4 ratio in patients with acute myocardial infarction (310). Altogether, it is not clear to what extent such SNPs contribute to local or systemic thyroid hormone regulation.

Genetic variation in other thyroid hormone transporters Several polymorphisms have been identified in genes encoding NTCP and the LATs. Some studies have shown that polymorphisms in NTCP can significantly diminish the uptake of bile 
acid in vitro (311). However, the presence of an association between these variants and serum thyroid hormone concentrations has not been studied thus far. Similarly, the impact of common genetic variants in LAT1 or LAT2 on serum thyroid hormone concentrations have not been studied, although one study has shown that the Chr16:87841130 G/C (p.Asn230Lys, rs1060250) variant in LAT1 does not affect the transport of phenylalanine (312).

A recent meta-analysis of genome-wide association studies showed that the Chr6:25777481 A/G (rs9356988) and Chr6:26001742 T/C (rs137964359) variants in the SLC17A4 locus are associated with serum FT4 concentrations in healthy individuals (12). Both variants are located outside the coding sequence, and it is currently unclear whether and how these variants affect SLC17A4 function. Alternatively, the effect may also be mediated through other variant(s) that are in high linkage disequilibrium.

\section{Monocarboxylate transporter 8 deficiency}

Historical background of monocarboxylate transporter $\mathbf{8}$ deficiency The relevance of thyroid hormone transporters became clear when the first patients with a genetic defect in MCT8 were identified. In 2004, Friesema and colleagues reported on patients with X-linked intellectual disability and abnormal thyroid function tests (low serum [F]T4, high [F]T3, low rT3, and high-normal TSH concentrations) associated with genetic mutations in the SLC16A2 gene (14). At the same time, Dumitrescu et al reported on 2 other families with very similar abnormalities who also harbored genetic mutations in SLC16A2 (15). Soon afterward it was realized that the neurological features of MCT8 deficiency were reminiscent of those described in 1944 by Allan et al, who published examples of a large family in which many male members were affected by intellectual disability (313). By reinvestigating this family more than 50 years later, Stevenson and Schwartz provided a detailed description of the clinical phenotype of this disorder, originally termed the Allan-Herndon syndrome and later Allan-Herndon-Dudley syndrome (AHDS), and localized the affected gene to the X chromosome $(314,315)$. Similarly, other families with this syndrome had been reported, in whom an X-linked mode of inheritance was suspected (eg, (316-319)). Genetic sequencing and the presence of abnormal serum thyroid function tests in these families confirmed that AHDS is caused by mutations in SLC16A2 (320, 321). Following the trends in the nomenclature of inherited disorders, AHDS is today preferentially termed after its disease-causing gene as MCT8 deficiency.

Genetic mutations in SLC16A2 At present, at least 320 affected individuals in 132 families have been reported in the literature (OrphaNet, Allan-Herndon-Dudley syndrome, OMIM 300523), with a broad variety of underlying genetic mutations (see Fig. 5) $(14,15$, 319-392). These mutations range from large deletions, with loss of one or more exons, frameshift deletions, single amino acid deletions, or insertions, to single amino acid substitutions, some of which have been identified in several unrelated patients (Fig. 5). Many of these mutations have been functionally evaluated in transiently or stably overexpressing cell lines and resulted in complete loss of MCT8 function, whereas a subset of mutations showed significant residual function $(80,324,340,366,393)$. According to these functional studies, roughly 3 different pathogenic mechanisms have been distinguished (Fig. 6). The first class of mutations alters the transporter kinetics, but not its expression levels at the cell membrane. These mutations can either partly (class 1A) or fully (class 1B) abrogate the MCT8-mediated transport of thyroid hormone. The second class comprises mutations that interfere with protein stability and/or trafficking toward the cell membrane. The level of residual activity of these mutant transporters can vary across different cell types. Missense mutations and the single amino acid deletions or insertions mainly group within the first 2 classes. The third class of mutations comprises the large deletions, premature stop mutations, and most of the frameshift mutations that fully inactivate the MCT8 protein regardless of the testing model. In addition to these in vitro overexpression studies, MCT8 was found to be the main thyroid hormone transporter in skin fibroblasts. For this reason, these cells have been used by several groups as an ex vivo model for MCT8 deficiency $(37,324,366)$. Both systems may yield a useful approach to predict the severity of neurocognitive disability, although a definitive genotype-phenotype relationship has not yet been established $(80,324,394,395)$. The available studies that have described the impact of the various mutations on MCT8 function have been recently extensively reviewed by Braun and Schweizer (255).

Clinical phenotype of monocarboxylate transporter 8 deficiency Through case descriptions published over the last 15 years, it became increasingly clear that the clinical phenotype of MCT8 deficiency comprises 2 major entities: 1) a neurocognitive phenotype and 2) signs of thyrotoxicosis in peripheral tissues, also termed the peripheral phenotype. The main findings of these case reports 


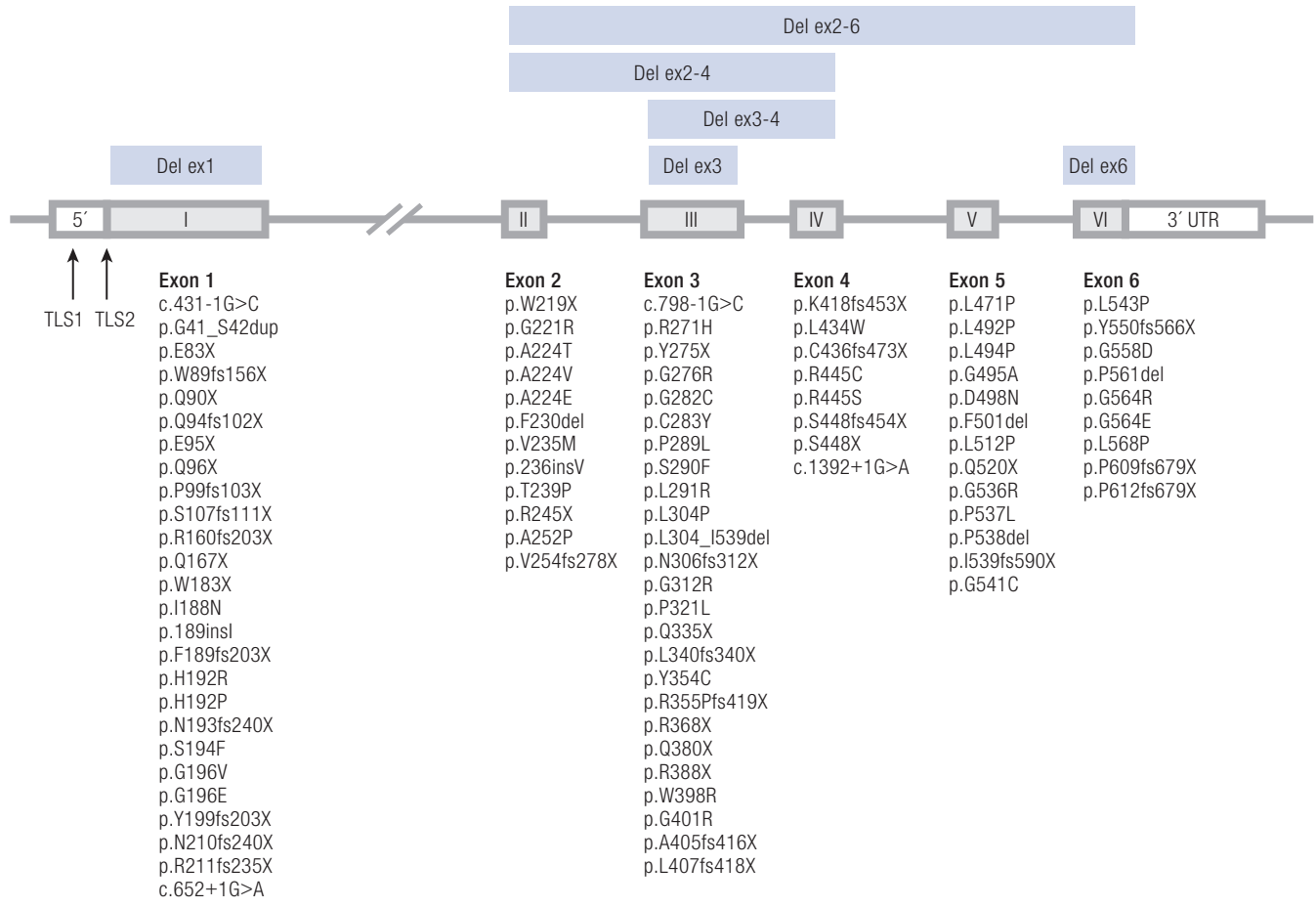

Figure 5. Schematic overview of the SLC16A2 gene that encodes MCT8. Indicated are the mutations identified in patients with MCT8 deficiency that have been reported in literature.

Type $1 \mathrm{~A}$

PM expression: normal

Transport function: (some) residual activity

Examples: p.R271H, Ins235V, p.L434W, p.G564E

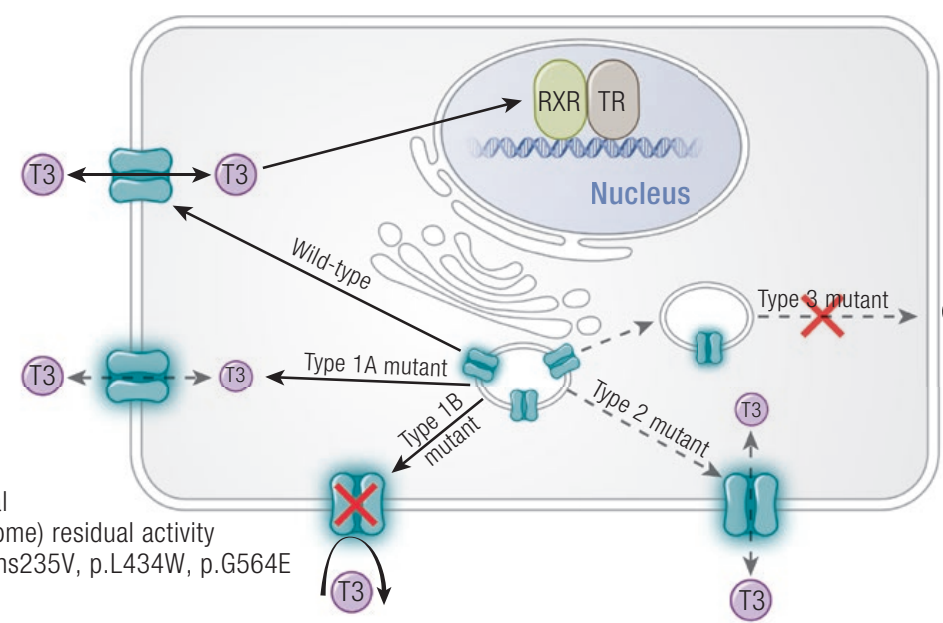

Type 1B

PM expression: normal

Transport function: no residual activity

Examples: p.Ins189I, p.F230del
Type 2

PM expression: reduced

Transport function: variable residual activity

Examples: p.G282C, p.F501del, p.L568P

Figure 6. Overview of pathogenic mechanisms of MCT8 mutations. Pathogenic mutations in MCT8 can be classified in 3 categories. Type 1 mutations do not affect cell membrane expression levels, but affect the affinity of MCT8 for its substrates or otherwise impede the translocation of substrates. Mutant proteins that belong to this class may exert some residual transport capacity (type 1A) or may be fully inactive (type 1B). Type 2 mutations predominantly affect MCT8 function by reducing protein stability or interfering with intracellular trafficking. These mutations result in reduced MCT8 protein levels at the plasma membrane (PM) or increased MCT8 turnover rate at the cell membrane. Several of the mutants that belong to this type of mutations have been shown to exhibit substantial transport activity once present at the cell membrane. Type 3 mutations mainly include premature stop or frameshift mutations and (large) deletions. These mutations are intrinsically inactive and may exhibit reduced protein expression levels. Of note, the residual transport capacity may vary between different cell models.

and series are summarized in Table 2 and some of the studies will be referred to in the following detailed descriptions of the clinical phenotype.
Pregnancies are uneventful, and children during fetal or early postnatal life do not present with overt abnormalities. Affected children typically present 
Table 2. Clinical Features of Monocarboxylate Transporter 88 Deficiency.

\begin{tabular}{|ll|}
\hline Neurological Phenotype & Peripheral Phenotype \\
\hline Clinical & Clinical \\
\hline Severe intellectual disability & $\begin{array}{c}\text { Progressive loss of body } \\
\text { weight }\end{array}$ \\
\hline $\begin{array}{l}\text { Failure to achieve motor } \\
\text { milestones }\end{array}$ & Muscle wasting \\
\hline Hypotonia & Increased perspiration \\
\hline Dystonia & Tachycardia \\
\hline Dyskinetic movements & \\
\hline Spasticity & Biochemical \\
\hline Brisk tendon reflexes & Elevated serum (F)T3 \\
\hline Contractures & Reduced serum (F)T4 \\
\hline Positive plantar & and r \\
\hline extension reflex & (High) normal serum \\
\hline Feeding problems & TSH \\
\hline Scoliosis & Elevated SHBG \\
\hline Dysmorphic features & \\
\hline MRI & \\
\hline Delayed myelination & \\
\hline Widened ventricles & \\
\hline
\end{tabular}

Abbreviations: MRI, magnetic resonance imaging; SHBG, sex hormone-binding globulin; TSH, thyrotropin.

with developmental delay and feeding problems in the first year of life. Most children do not attain full head control because of global hypotonia and do not develop any speech. During development, hypotonia persists and is manifested by poor head control, drooling, and swallowing problems. Affected children typically have increased muscle tone in the limbs and neck and exhibit abnormal body posturing, later on accompanied with clear signs of spasticity. Most patients are unable to sit independently and cannot walk, although some cases with a relatively less severe phenotype are able to walk, mostly with support, and have an ataxic gait $(321,340,366,392)$. The majority of patients reportedly have exaggerated tendon reflexes, contractures, and pathological plantar reflexes, suggesting the involvement of the pyramidal system. Based on the presence of these clinical features, patients have been classically described to exhibit spastic tetraplegia. However, in-depth neurological phenotyping studies by Matheus et al have attributed the peripheral hypertonia to dystonia rather than spasticity (396). Although signs of spasticity were detected, the authors stated that dystonic features prevail early in life. In retrospect, in some of the earlier studies the presence of dystonia and other dyskinetic movements have been mentioned, including athetosis, ataxia, and paroxysmal dyskinesia (361), whereas in others hypertonia reportedly varied and could be triggered by environmental cues, which better fits the definition of dyskinesia than spasticity. Therefore, MCT8 deficiency is increasingly regarded as a movement disorder. Intellectual disability is present in all patients. The severity of the neurocognitive phenotype varies, with a minority having a relatively less severe phenotype illustrated by the ability to talk in simple words and/or sentences. Several dysmorphic features have been documented, of which an elongated face, abnormal anatomy of the ears (large, cupped, or simple ears), chest malformations, and a myopathic facies have been reported most frequently. Despite a normal head circumference at birth, some patients present with microcephaly with advancing age. Seizures can be present, although these could be detected only by electroencephalography in a minority of cases. Evoked potentials are reportedly normal in some patients $(333,346,384,387)$ and delayed in others $(324,330,355,377,380,383,390)$. The key hallmark on brain magnetic resonance imaging (MRI) is delayed myelination, which has been described in almost all affected children younger than 5 years (eg, $(355,367,396))$. However, the progression of myelination to (near) normal in some older patients may help to discriminate MCT8 deficiency from other white-matter disorders such as leukodystrophies, cerebral palsy, and PelizaeusMerzbacher disease, with which MCT8 deficiency has been sometimes confused (330). Despite these notable improvements of myelination status on MRI, postmortem investigation of a brain from an 11-year-old patient with MCT8 deficiency indicated that in this patient myelination was still considerably delayed compared with healthy controls (358). Other frequently observed neuroradiological features are the presence of widened ventricles and a thin corpus callosum. More recent MRI studies using diffusion tensor imaging showed poor definition of especially the anteroposteriorly directed white-matter association tracks, which is consistent with the presence of dystonia (396).

The neurological features are accompanied by abnormal thyroid function tests. In most patients total T4 and FT4 concentrations are below the reference range, whereas the T3 concentrations 
are elevated. Like T4, rT3 concentrations also are decreased in most patients. Therefore, T3/(F) $\mathrm{T} 4$ and T3/rT3 ratios are strongly elevated. TSH concentrations are mostly within target range, but can also be mildly elevated (eg, (14)). In some patients, thyrotropin-releasing hormone (TRH)stimulation tests showed an inadequately low TSH response $(327,383)$. The elevated serum T3 concentrations may result in signs of hyperthyroidism in peripheral organs that rely on transporters other than MCT8.

Although it is imperative to recognize and treat the chronic thyrotoxicosis in the peripheral tissues of these patients, only a minority of the available case descriptions reported detailed information on the peripheral phenotype. Instead, most reports rather focused on the pronounced neurocognitive phenotype of MCT8 deficiency. Until recently, the lag in appropriate weight gain with advancing age and diminished muscle mass were the only features that had been widely documented. Both can possibly be attributed to the poor nutritional status due to difficulties in swallowing on a neurological basis, but also to the increased basal metabolism secondary to the hyperthyroid state in peripheral tissues $(261,341$, 379, 383). Linear growth appears less affected than body weight, typically resulting in very low body mass index scores. Despite being one of the most sensitive tissues for thyroid hormone, the cardiovascular system has not been widely studied in patients with MCT8 deficiency. Only a few patients were reported to display a high heart rate or (systolic) hypertension (330, 341, 362, $369,387)$, whereas some others exhibited no obvious abnormalities $(326,332,340,363,379,385)$. However, the majority of reports do not elaborate on these features. The presence of low total cholesterol and prealbumin concentrations, and elevated sex hormone-binding globulin (SHBG) concentrations described in some reports may indicate tissue hyperthyroidism in the liver (261, 332, 341, 352, 359, 362, 376, 379, 387).

A recent phenotypic characterization of a cohort of 46 patients as part of the baseline assessment of an interventional trial revealed that up to $43 \%$ of participants manifested tachycardia and 34\% systolic hypertension (397). Moreover, a substantial number of these patients experienced premature atrial complexes whose occurrence has been associated with hyperthyroidism and is highly abnormal in healthy children. In line with the available case reports and series, a lag in body weight gain was observed in most patients, with an average body weight for age $\mathrm{z}$ score of -2.84 ( \pm 1.88 ) and $65 \%$ of patients being underweight. The vast majority of patients in this study had elevated serum SHBG concentrations and low to normal serum creatinine concentrations, compatible with a hyperthyroid state in the liver and kidney. Being severely underweight and having cardiovascular dysfunction are thus important clinical sequelae of chronic peripheral thyrotoxicosis in MCT8 deficiency, both of which may pose important risk factors for premature death in MCT8 deficiency. Death during childhood is not uncommon, with (aspiration) pneumonia and sudden death being among the most frequently reported causes (330, 398). However, survival beyond age 70 years in some patients has been reported (321).

Future studies are warranted to uniformly characterize the clinical phenotype of MCT8 deficiency in greater detail. Such studies should aim to identify common medical problems in patients with MCT8 deficiency and determine the type and frequency of systematic clinical assessments and the need for symptomatic treatment.

\section{Diagnostic challenges in monocarboxylate trans- porter 8 deficiency.}

Diagnosing MCT8 deficiency can be challenging, especially because affected children typically present with unspecific signs and symptoms including failure to thrive and feeding problems, and the link with aberrant thyroid hormone action can be easily missed. The absence of clinical signs and symptoms that may help differentiate MCT8 deficiency from other neurodevelopmental disorders contributes to the diagnostic delay observed.

Therefore, aside from (next-generation) sequencing approaches, measurement of serum thyroid function tests would provide an important clue to properly diagnose MCT8 deficiency in the first year(s) of life. For this purpose, it is important to measure (F)T3 concentrations in addition to the routinely performed (F)T4 and TSH measurements to avoid misdiagnosis. The combination of low or low-to-normal (F)T4 concentrations and normal (to slightly elevated) TSH concentrations is often misinterpreted for secondary hypothyroidism, further delaying the diagnostic process. For similar reasons, MCT8 deficiency is unlikely to be detected with the currently employed neonatal screening programs for congenital hypothyroidism. In most countries, these programs are based on measurement of TSH concentrations alone, whereas in a small number of countries measuring T4 concentrations is the first step of the screening procedure. With either approach, the presence of normal TSH 
concentrations precludes affected children from being diagnosed with congenital hypothyroidism despite serum $\mathrm{T} 4$ concentrations being reportedly low in some affected neonates (366). It remains to be studied whether newborns with MCT8 deficiency already display the characteristic thyroid hormone profile and to what extent measurement of additional thyroid function tests during neonatal screening is technically and economically feasible. Although the pattern of the abnormal thyroid function tests is rather specific for MCT8 deficiency, a similar profile can be observed in patients with resistance to thyroid hormone (RTH) a due to mutations in THRA, encoding thyroid hormone receptor $\alpha$ (399).

Female carriers of genetic mutations in SLC16A2 Because the SLC16A2 gene is located on the $\mathrm{X}$ chromosome, mutations therein result in a clinical phenotype in male individuals. Female carriers typically do not present with phenotypic characteristics of MCT8 deficiency because they have a second, unaffected, copy of the SLC16A2 gene (334, 400). This results in the presence of functional MCT8 in at least a subset of cells. However, some female carriers reportedly have mild biochemical abnormalities that resemble those observed in affected male patients, and some of these carriers exhibit various degree of cognitive dysfunction (15, $321,340,342,379,401)$. This may be attributable to the presence of skewing in X-chromosome inactivation. Indeed, in one reported case, complete skewing of X-chromosome inactivation due an $\mathrm{X}$-autosome translocation resulted in the exclusive expression of the mutant MCT8 allele and a clinical phenotype resembling male MCT8 deficiency (365). Because the clinical and biochemical characteristics of female carriers have not been systematically described, the penetrance of such manifestations is currently unknown.

\section{Organic anion transporting polypeptide-1C1 deficiency}

Very recently, the first genetic defect in the brainspecific T4 transporter OATP1C1 was diagnosed through next-generation sequencing (16). This concerned a 15.5-year-old girl with apparent normal development in the first 2 years of life, who gradually developed autistic features and presented with progressive deterioration of cognitive and motor functions. In addition, she developed intolerance for cold. These clinical features were accompanied by gray- and white-matter degeneration and severe cerebral glucose hypometabolism. Serum free T4, free T3, and TSH concentrations were repeatedly within normal range. Exome sequencing identified a homozygous c.754G $>A$; p.D252N mutation that considerably diminished OATP1C1 transporter function in vitro.

The exact mechanisms underlying this disease are not known. However, several observations support the assumption that at least part of the phenotype can be ascribed to insufficient T4 uptake in OATP1C1-expressing astrocytes, which would lead to reduced availability of T3 to target cells within the CNS. At first, the presence of a strong reduction of brain glucose metabolism is compatible with a hypothyroid state of the brain (402). The presence of cerebral ataxia and autistic features have both been linked to a hypothyroid state in brain (403-405). In addition, the presence of subcortical white-matter atrophy may indicate deficiency of thyroid hormone in oligodendrocytes (406). Finally, treatment with the T3-analogue Triac appeared to halt the progression of the clinical course in the patient. The identification of additional cases will further advance the understanding of the mechanism of disease underlying OATP1C1 deficiency.

\section{Genetic defects in other thyroid hormone transporters}

Over recent years, genetic defects have also been identified in the SLC10A1 gene, encoding NTCP, and in the SLC7A5 gene, encoding the LAT1 light chain, and associated with clinical disorders.

Genetic defects in SLC10A1 have been identified in several patients with conjugated hypercholanemia (407-411). In at least one of these patients, serum thyroid function tests have been conducted that were reported to be normal (407). However, because NTCP predominantly transports sulfoconjugated iodothyronines, more extensive biochemical studies will provide additional insights into the effects of such mutations on thyroid hormone homeostasis, particularly in the liver.

Two different mutations in SLC7A5 have been identified in patients presenting with autism spectrum disorder and delayed development of motor and cognitive skills. Although both mutations diminish LAT1-mediated transport of leucine, their impact on iodothyronine transport has not yet been studied. Based on the phenotype of mouse mutants lacking Lat1 in endothelial cells of the $\mathrm{BBB}$, the authors concluded that the neurological impairments are most likely related to a compromised transport of branched chain amino acids into the brain (412) (see also "L-Amino Acid Transporters"). 


\section{Mechanisms of Disease-Monocarboxylate Transporter 8 and Organic Anion Transporting Polypeptide-1C1 Deficiency in Human and Animal Models}

The profound clinical symptoms of MCT8deficient patients provided the ultimate proof for the (patho-) physiological relevance of transportermediated thyroid hormone passage across cell membranes. At time of the identification of the first patients $(14,15)$, the exact mechanisms by which MCT8 deficiency evokes the characteristic changes in serum thyroid hormone parameters and-even more important-causes the severe neurological impairments were unclear. Tissue expression profiling in mice and human (see "Expression and Tissue Distribution of Monocarboxylate Transporter 8 and Monocarboxylate Transporter 10 ") as well as different animal models for human MCT8 deficiency have enhanced our understanding of the role of MCT8 in tissue thyroid hormone homeostasis, in particular in the HPT axis and brain (Fig. 8), and provided insights regarding the pathogenesis of MCT8 deficiency. Because the first patient with OATP1C1 deficiency has been identified only recently, the underlying pathophysiological mechanism is yet largely unclear.

The results of various animal models that have been established over the years to unravel the function of both transporters in the HPT axis, brain, and peripheral organs, are summarized in this section and in Table 3.

\section{Role of monocarboxylate transporter 8 within the hypothalamus-pituitary-thyroid axis}

MCT8 has been detected in neurons of the paraventricular hypothalamic nucleus in humans and rodents $(203,212)$. It is therefore tempting to speculate that MCT8 controls the negative feedback loop of the HPT axis by facilitating T3 entry into TRH-expressing neurons. In the human pituitary, MCT8 immunoreactivity was observed in folliculostellate cells that also express the thyroid hormone-activating enzyme DIO2. As such, these cells may control thyrotroph activity via a paracrine mode of action (425). More information is available regarding the localization and function of MCT8 in the human and mouse thyroid gland, where MCT8 is present at the basolateral membrane of follicular epithelial cells $(206,207)$. Therefore, it is conceivable that a global lack of MCT8 affects a proper function of the HPT axis on all levels as best illustrated in Mct8-ko mice (426).
By 2 different targeting strategies, Mct8deficient mouse models have been generated that exhibit the same characteristic features $(20,21)$. Mct8-ko mice show, like the patients, elevated serum T3, low T4, and low rT3 concentrations, whereas TSH concentrations are in the normal range or even slightly elevated. These mutant animals are therefore suitable to study the tissuespecific thyroidal state in the absence of MCT8. Rather complex alterations were found that can be either ascribed to the thyrotoxic effects of excessive circulating thyroid hormone concentrations, or to the impaired tissue-specific cellular transport of thyroid hormone. TRH mRNA expression in the hypothalamus in these Mct8-ko animals was strongly elevated, indicative of a pronounced thyroid hormone-deficient state in thyroid hormonesensitive hypothalamic neurons. By contrast, the thyroid hormone content of the thyroid gland was highly elevated, pointing to tissue-specific thyroid hormone excess $(20,206,207)$. Moreover, circulating thyroid hormone concentrations are not properly sensed on the hypothalamus as well as on the pituitary levels as illustrated by TSH suppression tests $(20,21)$. A similar observation has been described in one MCT8-deficient patient in whom only T4 but not T3 was able to suppress TSH (418), indicating a comparable function of MCT8 in the murine and human HPT axis. Consequently, serum TSH values are not a valid read-out parameter for assessing the general thyroid hormone status in MCT8-deficient patients because the hypothalamus and pituitary are relatively insensitive toward thyroid hormones.

Beyond age 6 to 12 months, Mct8-ko mice develop structural abnormalities in the thyroid gland with features reminiscent of papillary microcarcinoma, and a similar observation has been made in one MCT8 patient (418). The driving factor for these morphological changes still needs to be unraveled, and it is yet unclear whether this is a common feature of MCT8 deficiency.

A key problem that is still not completely resolved is the exact pathogenic mechanism that underlies the highly increased serum T3:T4 ratio. Over the years many hypotheses on this matter have been generated, but most of them have been partly refuted. A disturbed thyroid hormone metabolism, in particular a rise in $\mathrm{T} 4$ to $\mathrm{T} 3$ conversion, represents a likely explanation, and in fact, DIO1 and DIO2 activities were found to be altered in various tissues of Mct8-ko mice. Notably, DIO1 activities are strongly increased in the liver and kidneys, whereas DIO2 activities were found 

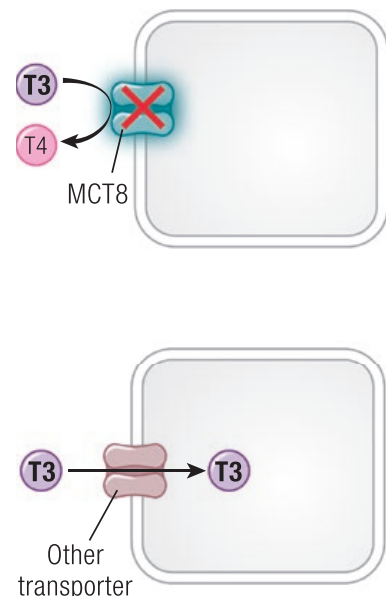

\section{Hypothyroid}

CNS - developmental delay, intellectual disability

Pituitary - inappropriate TSH

\section{Hyperthyroid}

General - low body weight, increased perspiration

Cardiovascular - tachycardia, premature atrial complexes,

hypertension

Liver - increased SHBG, low cholesterol

Muscle - muscle wasting, low creatinine

Kidney - low creatinine

Figure 7. Schematic representation of the pathophysiology of MCT8 deficiency. The current paradigm of MCT8 deficiency holds that tissues that largely rely on MCT8 for cellular thyroid hormone uptake, including the brain, reside in a hypothyroid state, whereas tissues that rely on transporters other than MCT8 reside in a hyperthyroid state due to exposure to the elevated serum T3 concentrations. This results in an impaired brain development due to brain hypothyroidism and signs of tissue hyperthyroidism in peripheral organs. CNS indicates central nervous system. Abbreviations: SHBG, sex hormone-binding globulin; TSH, thyrotropin.

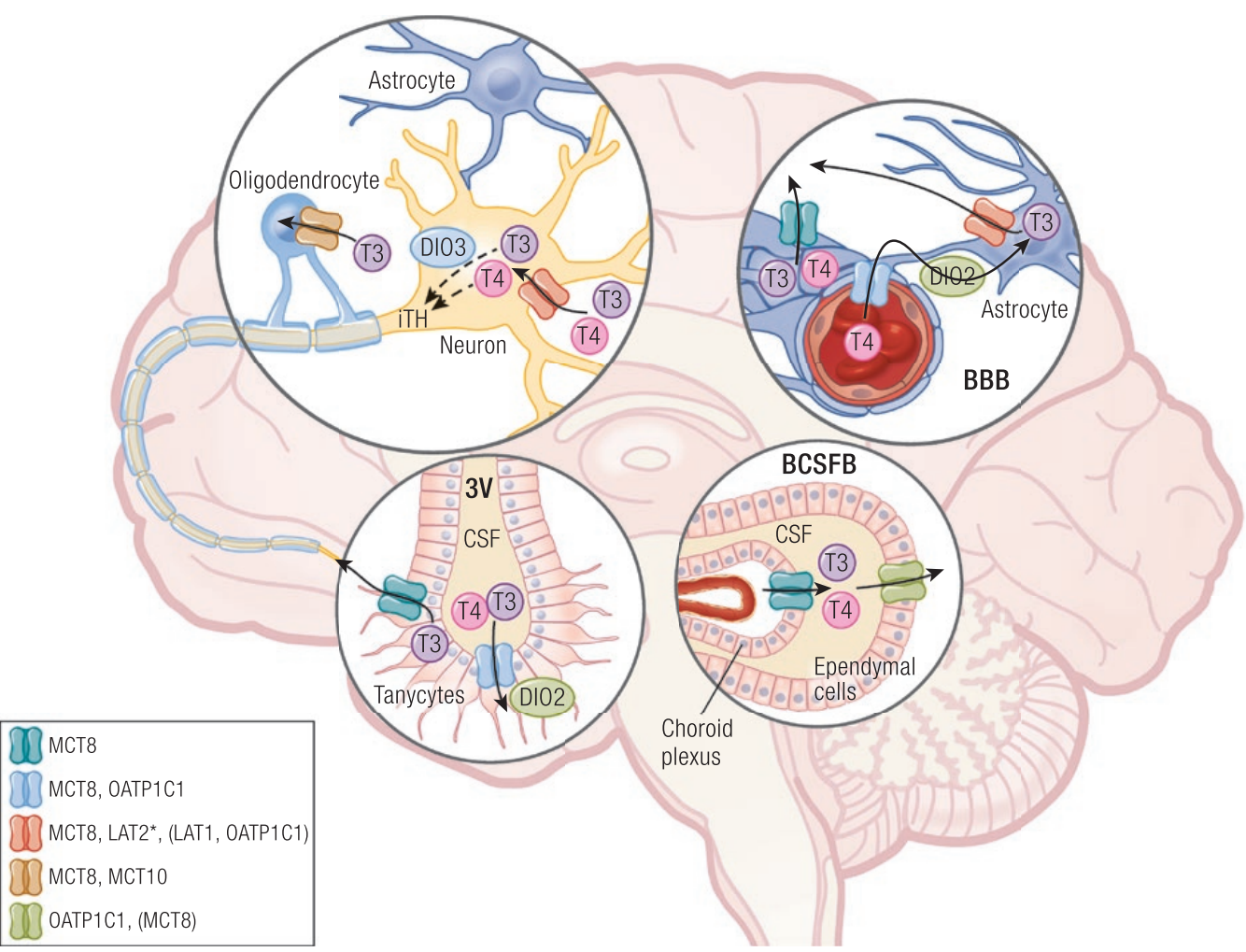

Figure 8. A putative model of thyroid hormone signaling in the human brain based on the available studies. To exert its effects inside the brain, thyroid hormone needs to cross multiple barriers. This illustration provides a schematic overview of the different barriers in the human brain in which MCT8 and OATP1C1, and possibly additional transporters, might be involved in the transport of thyroid hormone. In most cases definite proof of functional contribution to thyroid hormone transport is lacking. Moreover, the expression and contribution of the various thyroid hormone transporters likely depend on the developmental stage and vary between different cell populations of the same tissue. It is also unclear to what extent transporters other than those displayed here are expressed at the protein level at the different sites and contribute to thyroid hormone transport in the human brain. * LAT2 is localized to adult neurons and microglia, but is absent in fetal neurons. Recently proposed novel routes by which thyroid hormone may enter the brain through the inner and outer cerebrospinal fluid brain barrier are not depicted. Abbreviations: $3 \mathrm{~V}$, third ventricle; $\mathrm{BBB}$, blood-brain barrier; $\mathrm{BCSFB}$, blood-cerebrospinal fluid barrier; CSF, cerebrospinal fluid; iTH, inactivated thyroid hormones. 


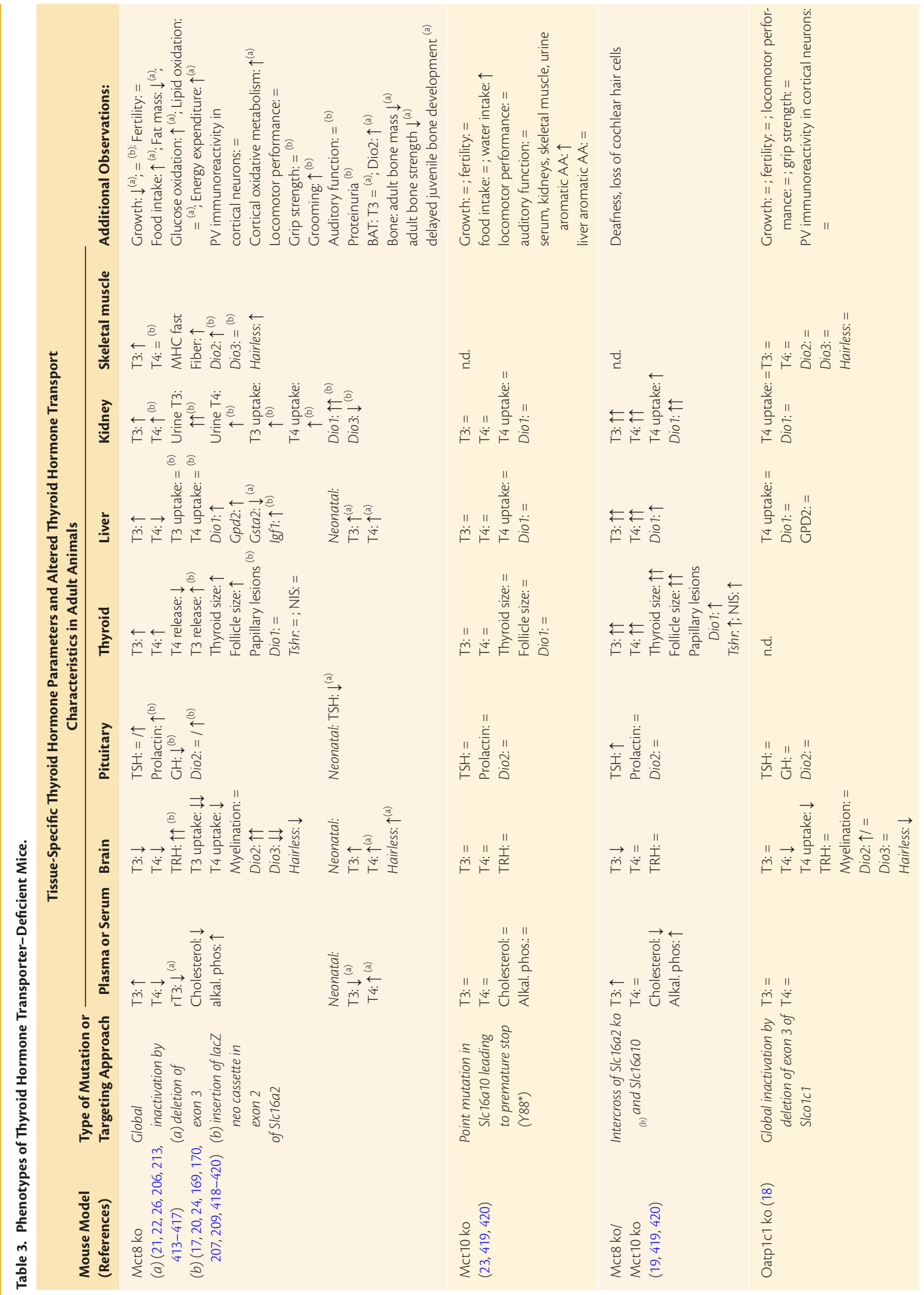




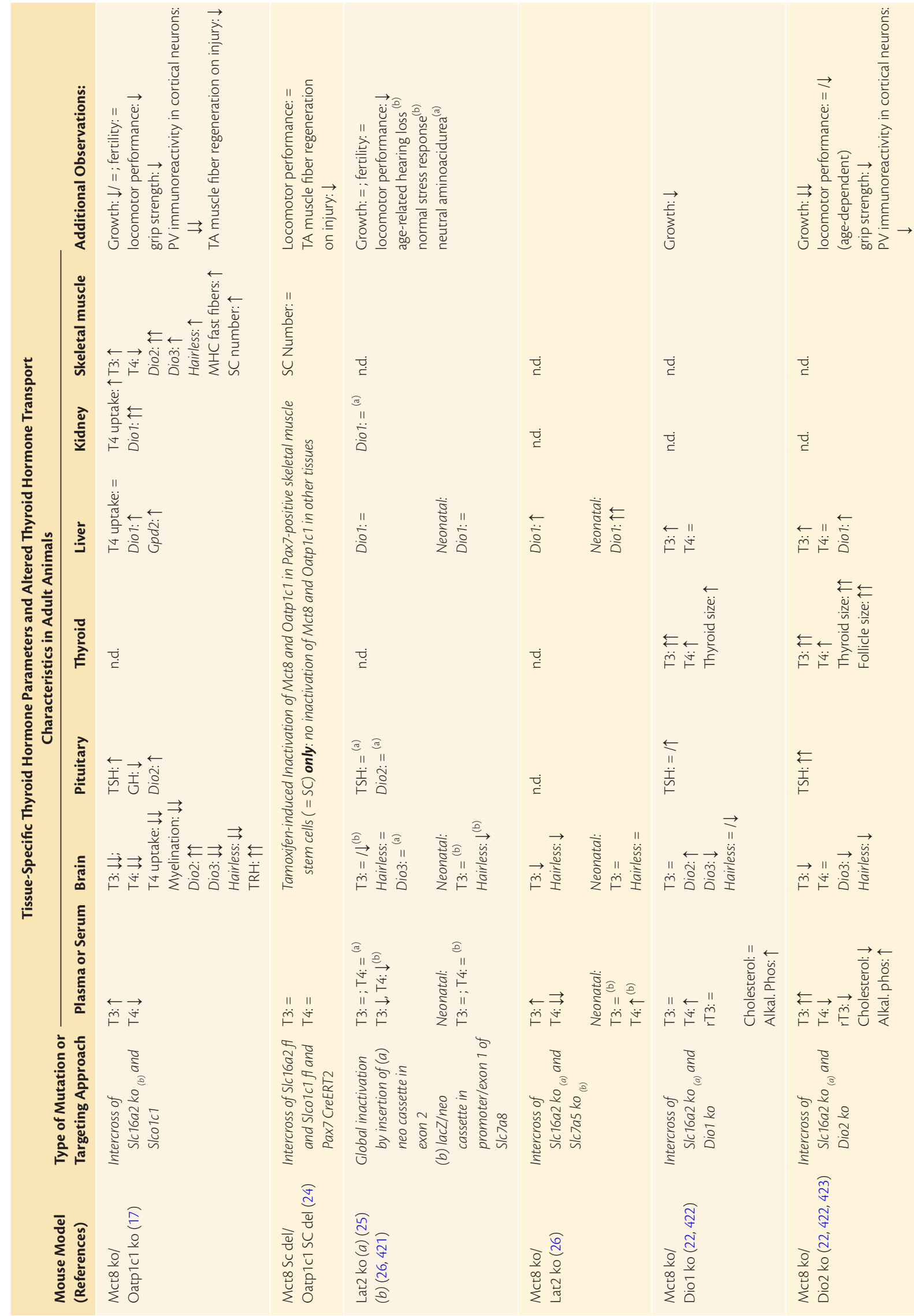




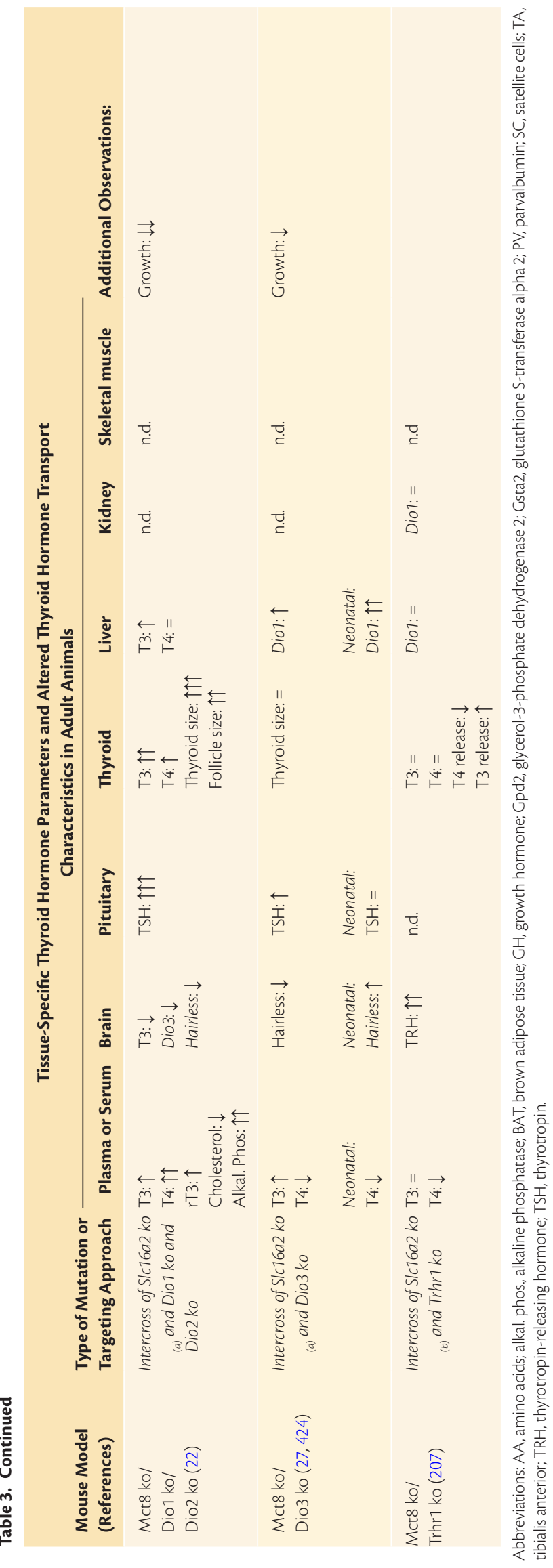

to be elevated in the brain, pituitary, and skeletal muscle (20, 21, 24). To pin down the responsible deiodinase, Liao et al generated Mct8/Dio1 and Mct8/Dio2 double-mutant mice and were able to demonstrate by this elegant approach that DIO1 is primarily responsible for the abnormal T3:T4 ratio in Mct8-ko mice (22). Still, it needs to be disclosed why DIO1 is elevated in MCT8-deficient mice and which tissue is critically involved in generating the high serum T3 concentrations. Given the pronounced expression of MCT8 along the HPT axis, it was reasoned that the reduced uptake of T3 into the brain and pituitary cells may result in a progressive buildup in serum, resulting in stimulation of DIO1 in the liver and kidney. This hypothesis was ruled out by studying athyroid Mct8/Pax8 double-ko (dko) animals and Pax8 ko mice that both do not produce any thyroid hormone endogenously. When both animal groups were exogenously treated with $\mathrm{T} 3$, serum $\mathrm{T} 3$ concentrations were found to be similar. These findings indicate that an impaired T3 entry in the brain does not cause elevated serum T3 levels in Mct8-ko mice (207). However, treatment of Mct8/Pax8-dko and Pax8-ko mice with the same dose of L-T4 caused reduced T4 concentrations in Mct8/Pax8-dko animals only (207). Thus, enhanced T4 metabolisms in other tissue (most likely in the kidneys) contributes to the abnormal T3:T4 ratio characteristic of MCT8 deficiency (see "Role of MCT8 in Peripheral Organs").

A second line of evidence points to a contributing role of the thyroid gland itself. TSH stimulation tests of Mct8-ko mice revealed a reduced $\mathrm{T} 4$ secretion indicating that Mct8 contributes significantly to the T4 efflux in thyrocytes. To what extent the MCT8-deficient thyroid gland also shows increased T3 secretion is still a matter of debate because conflicting data have been published $(206,207)$. This issue will ultimately be clarified by the generation and analysis of mouse mutants that selectively lack MCT8 in the thyroid gland.

Altogether, studies of Mct8 mutant mice have provided valuable insights into the changes in thyroid hormone metabolism and action within the HPT axis and importantly guided therapeutic approaches in patients with MCT8 deficiency (see "Therapeutic Approaches in Thyroid Hormone Transporter Defects").

\section{Role of monocarboxylate transporter 8 in peripheral organs}

Apart from the HPT axis, various organs show alterations in tissue-specific thyroid hormone status in the absence of MCT8. Mct8-ko mice have an increased liver T3 content that is accompanied by increased DIO1 expression levels and activity, as well as reduced serum cholesterol concentrations (21). This is in line with changes observed in serum markers that reflect liver thyroid hormone action in human (see "Clinical Phenotype of MCT8 Deficiency").

Similar to the liver, kidneys of Mct8-ko mice were found to be in a hyperthyroid state as evidenced by a pronounced increased thyroid hormone content and a steep rise in DIO1 activity that even exceeded levels found in liver homogenates. In situ hybridization (ISH) studies revealed that renal Dio1 expression is specifically elevated in proximal tubule cells that under normal 
conditions express MCT8 (209). Strikingly, in vivo transport studies revealed an increased accumulation of peripherally injected radiolabeled T3 and T4 in kidney but not in liver homogenates of Mct8-ko mice. It is therefore tempting to speculate that in kidneys, MCT8 represents a critical thyroid hormone efflux system whereas in liver, absence of MCT8 has very little effect on thyroid hormone passage presumably due to the vast repertoire of additional thyroid hormone transport systems that fully compensates for the absence of MCT8 in liver. Moreover, absence of MCT8 in the liver is not likely to be the primary cause of the altered serum thyroid hormone profile due to stimulation of DIO1, as liver-specific inactivation of Dio1 in global Mct8ko mice did not restore normal circulating thyroid hormone concentrations (427). The physiological importance of renal thyroid hormone metabolism in the control of systemic thyroid hormone homeostasis has not been fully clarified as yet, although recent studies suggest that murine kidneys may be the major storage site for (glucuronidated) $\mathrm{T} 4$ (428). It is therefore conceivable that because of a compromised renal T4 efflux in MCT8 deficiency, T4 is trapped in the kidneys and that this intrarenal accumulation of T4, together with the steep rise in renal DIO1 activity, represents the key event for generating the abnormal serum thyroid hormone parameters. This hypothesis, however, needs to be confirmed by generating kidney-specific Mct8-ko animals.

Similar to patients with MCT8 deficiency, Mct8-ko mice show enhanced energy expenditure and reduced fat mass (413). Despite the absence of MCT8, brown adipose tissue (BAT) obviously senses the drop in circulating T4 but not the high T3 concentrations as evidenced by normal-tissue T3 content as well as the gene expression profile in BAT of Mct8-ko mice (413). In contrast, skeletal muscle fully senses the increased serum $\mathrm{T} 33$ concentrations and shows increased muscle $\mathrm{T} 3$ content along with anticipated changes in T3-regulated gene expression patterns of myosin heavy chains. Furthermore, analysis of muscle fiber composition in different skeletal muscle subtypes indicates a shift from slowtwitch to fast-twitch fibers as expected from hyperthyroid muscle tissue $(24,413)$.

Postnatal skeletal development and bone maintenance is compromised in Mct8-ko mice as well (414). Delayed endochondral ossification and a transient growth retardation point to a thyroid hormone-deficient situation in growth plate chondrocytes of juvenile Mct8-ko mice. By contrast, decreased bone mass and mineralization together with reduced bone strength are observed in adult MCT8-deficient mice and are typical changes seen under hyperthyroid conditions. Obviously, mature osteocytes, osteoblasts, and/or osteoclasts express additional thyroid hormone transporters and are therefore supplied with increased concentrations of $\mathrm{T} 3$ from the circulation. The thyroid hormone status in bone of human patients with defective MCT8 is currently unknown, because bone-turnover markers are difficult to interpret in the context of their immobility.

The similarities between the "peripheral phenotype" of Mct8-ko mice and the set of clinical data obtained from MCT8 patients thus support the conclusion that the Mct8-ko mouse is indeed a useful model to study the pathophysiological events underlying the abnormalities in thyroid hormone homeostasis in the HPT axis and peripheral organs. Together, these observations emphasize the need to restore the abnormally high serum T3 concentrations in patients with MCT8 deficiency to alleviate tissue thyrotoxicosis in peripheral organs. These tissues likely rely on transporters other than MCT8 and as such are exposed to the high serum T3 concentrations (Fig. 7).

\section{Role of monocarboxylate transporter 8 in the brain}

Characteristic features of a monocarboxylate transporter 8-deficient central nervous system The presence of severe intellectual and motor disability in patients with MCT8 deficiency suggests that functional MCT8 is crucial for normal brain development. In addition to the clinical and neuroradiological manifestations (see "Clinical Phenotype of MCT8 Deficiency"), López-Espíndola et al reported the first description of neuropathological abnormalities in MCT8deficient cerebral cortex and cerebellum derived from a male fetus (GW30) and an 11-year-old boy (358). In the fetus, a delay in cortical and cerebellar development was noted that was accompanied by diminished myelination, decreased expression of the calcium-binding protein parvalbumin, and impaired axonal maturation. These findings suggest that brain abnormalities may be present during fetal development and involve both neurons and glia cells. Similarly, reduced myelination and disturbed cerebellar development was noted in the 11-year-old MCT8-deficient patient. All of these features have been described in hypothyroid rodent brains and are thus characteristic of a thyroid hormone-deficient state. Indeed, determination of thyroid hormone tissue concentrations confirmed 
that $\mathrm{T} 4$ concentrations in the fetal cortex were reduced by $50 \%$.

In contrast to the histomorphological changes observed in patients with MCT8 deficiency, the brains of Mct8-ko mice are surprisingly normal. Though mildly reduced brain thyroid hormone content indicates a modest degree of thyroid hormone deficiency within the CNS, brain structures and neuronal functions appear to be unaffected. For instance, these mice did not show an ataxic gait, a typical sign of aberrant cerebellar development resulting from insufficient thyroid hormone signaling. These observations questioned a pivotal function of MCT8 in the mouse brain. Only increased metabolic activity was detected by carbon-13 nuclear magnetic resonance spectroscopy in brain homogenates of Mct8-ko mice. Yet, how this phenotype relates to possible changes in thyroid hormone homeostasis is still not understood (415). Interestingly, in vivo transport studies of Mct8-ko mice disclosed a highly diminished transfer of T3 into the brain, whereas T4 transport was only partially affected (213). The lack of T3 uptake in the absence of MCT8 can apparently be compensated for by increased DIO2 activity, and the amount of locally generated $\mathrm{T} 3$ appears to be sufficient to sustain normal brain development in Mct8-ko mice. Indeed, Mct8/Dio2-dkomice showed a hypothyroid brain and accompanying neuromotor abnormalities (423).

The presence of substantial T4 concentrations in the mouse brain in the absence of MCT8 had been attributed to the presence of alternative T4 transporters at critical sites in mice that are absent in human. Indeed, Roberts and colleagues demonstrated that the T4 transporter OATP1C1 is present in the brain microvessels of rodents, but not in those of humans (108). The relevance of this transporter in mice was underscored by the Mct8/Oatp1c1-dko mice, which showed highly reduced brain thyroid hormone content due to a strongly diminished blood to brain passage of both T4 and T3. By contrast, T4 transport into the CNS is reduced to only $50 \%$ of WT levels in Mct8 or Oatp1c1 single-ko mouse (17). Unlike the single thyroid hormone transporter mutant mice, the Mct8/Oatp1c1-dko animals showed similar histological abnormalities as seen in patients with MCT8 deficiency, including delayed cerebellar development, compromised maturation of cortical GABAergic interneurons, and persistent hypomyelination. These structural hallmarks are indicative of a widespread thyroid hormone deficiency in the CNS that was confirmed by assessing gene expression of well-established thyroid hormone-regulated target genes. Moreover, Mct8/ Oatp1c1-dko mice exhibit pronounced locomotor impairments, learning disabilities, reduced muscle strength, and gait abnormalities. In light of these findings, Mct8/Oatp1c1-dko mice have been suggested as a more adequate mouse model for human MCT8 deficiency compared to the single Mct8-ko mouse, with its rather normal brain development. Importantly, these observations highlight that differences in tissue distribution and cellspecific function of thyroid hormone transporters among species may determine the cell-specific vulnerability toward thyroid hormone transporter defects.

The zebrafish represents another species in which brain function of MCT8 has been extensively studied $(218,429)$. Induction of $m c t 8$-knockdown by morpholino injection resulted in larvae exhibiting a reduced brain size, a curved body axis with a deformed spinal cord, and impaired mobility $(29,31,215)$. Reduced locomotor activity was fully replicated in Mct8-deficient zebrafish generated by zinc finger nuclease technology (216). Moreover, Mct8-deficient zebrafish showed neurological and behavioral deficiencies together with hypomyelination and a reduced number of oligodendrocytes, disturbed axonal branching of sensory neurons, as well as reduced synaptic density of motor neurons $(30,216)$. Based on these profound alterations, Mct8-deficient zebrafish can be considered as a model system for MCT8 deficiency as well.

\section{Role of monocarboxylate transporter 8 in neurons} The seeming lack of any overt neuronal damage in Mct8-ko mice shed new light on the function of MCT8 in neurons and, at least in mice, questioned its role as a critical neuronal T3 uptake system. Though murine neurons lacking MCT8 exhibited decreased T3 transport in primary cortical cultures, MCT8-deficient cerebellar Purkinje cells responded quite normally to T3 treatment in vitro $(20,169)$. Thus, at least in mice, the majority of Mct8-deficient neurons are obviously still capable of importing T3, indicating that additional T3 transport systems must be present to ensure sufficient T3 supply. Based on the overlapping expression patterns of Mct8 and Lat2 in the mouse brain, it had been postulated that LAT2-mediated T3 transport may compensate for a loss of MCT8 in neurons (169). However, studies of Mct8/Lat2dko mice refuted this assumption because apart from minor neonatal alterations in cortical thyroid hormone signaling, these mice exhibited a phenotype similar to single Mct8-ko animals (26). 
Further in-depth studies are therefore needed to identify additional neuronal T3 transport systems that may act in concert with MCT8.

It is unclear whether this also applies to human neurons. Patient-derived iPSCs have been successfully differentiated into neural cells that indeed exhibited a reduced thyroid hormone transport compared to MCT8-expressing cells. However, their differentiation potential toward neuronal progenitors and beta III-tubulin-expressing neurons was not greatly compromised, suggesting that sufficient amounts of T3 were imported even in the absence of MCT8 under the conditions tested (28). Recently, MCT8 immunoreactivity in cortical neurons was found to increase after GW32, which may support a minor role for MCT8 in earlier stages of neuronal development in humans. Moreover, MCT8 was detected in radial glia cells, suggesting a critical function during neuronal specification and/or differentiation (58).

Only in a nonmammalian species, could neuronal thyroid hormone transport be clearly attributed to MCT8. In the embryonic chicken, downregulation of MCT8 by RNA interference in cerebellar Purkinje cells resulted in disturbed dendritogenesis and caused delayed cerebellar development, both of which are a classical hallmark of a hypothyroid developing cerebellum (32). In developing optic tectum, a structure in the chicken brain that shares many features with the mammalian cerebral cortex, downregulation of MCT8 in neural progenitors compromises neurogenesis and affects neuronal migration, both processes that rely on proper thyroid hormone signaling as well (429, 430). Also in chicken retinal precursor cells, MCT8 is crucial for normal development (431). It remains to be investigated, however, to what extent chicken MCT8 contributes to T3 transport in neurons at a later, more mature stage.

Role of monocarboxylate transporter 8 at brain barriers The severely impaired passage of T3 from blood to brain in Mct8-ko mice supports a critical function of MCT8 at the BBB and/or the BCSFB. Indeed, these brain barriers already restrict the paracellular passage of low-molecular-weight molecules during very early stages of development (432). It is therefore likely that also thyroid hormone requires transporter proteins to facilitate transcellular passage across brain barriers during prenatal stages of brain development.

The BCSFB consists of a monolayer of choroid plexus epithelial cells that are equipped with tight junctions and are located in the third, fourth, and lateral ventricles of the brain, where they are responsible for CSF production. Choroid plexus epithelial cells surround fenestrated capillaries and are directly connected to the ventricle ependymal cells that together comprise the ventricle wall. In rodents and chickens, MCT8 is present both at the apical and basal membrane of choroid plexus epithelial cells $(164,212,217,433)$. In the human fetal and adult brain, MCT8 was detected in the epithelium of the choroid plexus and ependymal cells as well $(58,108,220)$. Based on the presence of MCT8 in radial glial cells (see "Expression and Tissue Distribution of Monocarboxylate Transporter 8 and Monocarboxylate Transporter 10"), it has been recently postulated that these cells may form another route via which thyroid hormones may enter the brain during embryonic development. To what extent transfer of thyroid hormone across these barriers is physiologically relevant is still a matter of debate because experimental data from rodents question a pivotal function of this transport route. In a seminal study, Dratman et al demonstrated by radioautography that radiolabeled thyroid hormone delivered to the CSF of adult rats only penetrated the periventricular areas of brain parenchyma, whereas peripherally applied thyroid hormone entered all brain regions (434). Importantly, $\mathrm{DIO} 2$ and DIO3 expression is relatively weak in the BBB compared to the other brain barriers (58). Although these findings point to the $\mathrm{BBB}$ as the predominant pathway for thyroid hormone entry into CNS, the situation may be completely different at prenatal and early postnatal stages, when the ventricular system is disproportionally larger and comprises much more space. However, information on thyroid hormone concentrations in the CSF is very limited. The few available studies may support a role for MCT8 in the transport of thyroid hormone across the BCSFB and ependymal cells of the ventricular wall, at least during distinct developmental time periods (375).

Of note, expression of the thyroid hormonebinding protein transthyretin (TTR), produced by choroid plexus cells, was found to be at its highest in the CSF during the second trimester of pregnancy, when the human fetal brain needs increased amount of thyroid hormone for proper neural maturation. The exact role of TTR in the transport of thyroid hormone across the brain barriers and in the distribution of thyroid hormone throughout the brain is yet unclear. It appears unlikely that thyroid hormone enters the brain by crossing the $\mathrm{BBB}$ or choroid plexus while remaining in complex with TTR (435). Rather, locally produced TTR may create a favorable gradient for the efflux of thyroid hormone from the vascular endothelial cells or 
choroid plexus epithelial cells into the brain and help target thyroid hormone to specific neuronal populations (436-438).

At the $\mathrm{BBB}$, specialized endothelial cells form tight junctions to restrict paracellular diffusion. Vascular permeability is further determined by pericytes covering the blood vessels and contributing to the formation of the basal membrane. In the mature CNS, astrocytic end feet encircle almost the entire surface of endothelial cells and are believed to control the barrier properties of the endothelial cells. In human as well as rodent brain, immunohistochemical studies clearly demonstrated the presence of MCT8 in capillary endothelial cells $(58,108)$. Endothelial expression of Mct8 was also observed in RNA sequencing (RNAseq) experiments using fluorescenceactivated cell sorter- (FACS-) sorted cells derived from 7 -day-old mouse brains $(4,439)$. Proteomic profiling of endothelial cells derived from primates confirmed an endothelial localization of MCT8 as well (109). Proof of the functional relevance of MCT8 in human endothelial cells was ultimately provided by in vitro studies using iPSC-derived BMECs. Indeed, MCT8-deficient BMECs cultured in specialized wells to induce BBB-like features exhibited a reduced T3 and T4 uptake as well as efflux compared to MCT8-expressing control cells. Remarkably, only the reported net transcellular transport of T3, and not T4, from the blood to the brain compartment of this BBB model system was significantly reduced in the absence of functional MCT8 (28).

In the zebrafish, Mct8 seems to mediate T3 transport across the $\mathrm{BBB}$ as well. A functional barrier in zebrafish is first detected in 3-days postfertilization (dpf) embryos and its maturation occurs between 3 and $10 \mathrm{dpf}(440,441)$. Interestingly, Mct8-deficient zebrafish larvae treated prior to $3 \mathrm{dpf}$ with $\mathrm{T} 3$ showed a normal number of oligodendrocytes, whereas application of T3 after maturation of the BBB was ineffective. Likewise, reexpression of Mct8 in the vascular and BBB system only was sufficient to restore normal oligodendrocyte numbers (30). These data imply that also in zebrafish $\mathrm{T} 3$ requires the function of Mct8 at the BBB for entering the CNS.

Role of monocarboxylate transporter $\mathbf{8}$ in glia cells In comparison to neurons, endothelial cells, and choroid plexus structures, localization of MCT8 in glia cells is less well investigated. Though RNAseq data confirmed the presence of Mct8 transcripts in FACS-sorted mouse astrocytes (439), ISH studies failed to detect any coexpression of Mct8 and
Gfap, an astrocytic marker, in the hippocampus of 21-day-old mice (212). Most likely, Mct8 expression is present only in a subset of astrocytes. In humans, RNAseq analysis of cortical astrocytes derived from fetal (17-20 GW) or mature (ages 8-63 years) brain samples and purified by immune-panning revealed MCT8 expression in both cell populations (442). In human brain samples, astrocytes around vascular cells in the BBB positively stained for MCT8 (58). It also remains to be investigated to what extent other subtypes of astrocytes depend on MCT8 for thyroid hormone import and export.

It is well established that thyroid hormone plays a major role in myelination (443). T3 strongly stimulates the differentiation of oligodendrocyte precursor cells by triggering cell-cycle arrest and inducing the expression of premyelinating genes that in turn promotes the differentiation of oligodendrocyte precursor cells into myelinating oligodendrocytes. Moreover, thyroid hormone regulates the expression of practically all main myelin proteins, including proteolipid protein, myelin basic protein, and myelin-associated glycoprotein. Thus, the delayed myelination in the CNS of MCT8-deficient patients could be interpreted as a direct reflection of the overall reduced brain thyroid hormone content due to an impaired transport across the $\mathrm{BBB}$, although reduced myelination secondary to poor neuronal development cannot be excluded. Apart from its pivotal role at the BBB, MCT8 may also be needed for the import of T3 into oligodendrocytes and/or its precursor cells (219), which needs to be studied in more detail.

\section{Physiological relevance of other thyroid hormone transporters}

Apart from MCT8, other transport proteins are able to facilitate thyroid hormone transmembrane passage in vitro, as have been discussed in "Thyroid Hormone Transporter Families and Their Molecular Characteristics." Yet, for only a few of them could a physiologically relevant contribution in tissue-specific thyroid hormone transport be firmly established.

\section{Organic anion transporting polypeptide-1C1}

In rodents, OATP1C1 is highly expressed in the brain, where it was detected in capillary endothelial cells, choroid plexus structures, and astrocytes (see "Organic anion transporting polypeptides-1C1"). The presence of functional OATP1C1 has been confirmed in a subset of astrocytes by sulforhodamine 101 tracing, a fluorophore that is selectively transported by 
OATP1C1 (444). Given its very restricted expression pattern, it is not surprising that Oatp1c1-ko mice do not show any alterations in serum thyroid hormone profile nor any signs of altered thyroid hormone content in peripheral tissues (18). However, brain T4 content of Oatp1c1-ko mice was decreased to around $60 \%$ compared to healthy littermates, which is likely attributable to a reduction in blood-brain T4 transport $(17,18)$. Notably, DIO2 activities in the brain were elevated 3-fold, indicating that DIO2-expressing cells (eg, astrocytes (4)) are in a T4-deficient state. As a consequence, brain T3 signaling was only slightly reduced in Oatp1c1-ko mice, presumably due to increased local T3 production and the presence of MCT8, which ensures normal T3 transport across brain barriers in these animals. The latter was indeed supported by the clear presence of brain hypothyroidism in mice lacking both OATP1C1 and MCT8, as described in more detail in "Characteristic features of a monocarboxylate transporter 8-deficient central nervous system." Thus, these observations suggest that in rodents, OATP $1 \mathrm{C} 1$ is particularly important for the transport of $\mathrm{T} 4$ across the brain barriers and into astrocytes.

However, OATP1C1 is also present in neuronal precursor cells during development, as illustrated in mice carrying a cre-recombinase inserted into the Oatp1c1 (Slco1c1) locus (445). At E15, beta-galactosidase staining was present in TBR2positive neuronal progenitor cells that give rise to cortical neurons of layer $2 / 3$. Thus, even though global Oatp1c1-ko mice do not show any cortical abnormality or behavioral alterations, it still remains to be investigated to what extent MCT8 and OATP1C1 cooperate in neuronal thyroid hormone transport during embryonic stages and to what extent combined MCT8/OATP1C1 deficiency in neuronal precursor cells compromise their differentiation.

OATP1C1 is also expressed in the human CNS, where it apparently is critical for normal CNS function as evidenced by the clinical findings of an OATP1C1-deficient patient (16). However, as the rodent brain suggests, the human CNS is more dependent on a functional MCT8 at the BBB and/or BCSFB than the rodent brain, in which absence of MCT8 can be at least partially compensated for by OATP1C1. Given its presence at the inner and outer CSFBB, OATP1C1 may also contribute to the transport of thyroid hormone into the brain via these routes. OATP1C1 was also localized in radial glial cells as well as astrocytes surrounding the vascular endothelial cells of the BBB, where OATP1C1, together with MCT8, facilitates thyroid hormone uptake (110). However, the exact contribution of the individual transporters to the thyroid hormone transport in these and other astroglial cells remains to be resolved.

Outside the CNS, information regarding the function of OATP1C1 is very scarce. A distinct function of MCT8 and OATP1C1 has been found in murine satellite cells. These stem cells of the skeletal muscle express OATP1C1 only temporarily when they become activated on muscle injury (24). Indeed, skeletal muscle tissue lacking MCT8 and OATP1C1 only in satellite cells shows a compromised regeneration capacity on an injury, because satellite cell differentiation requires a fine-tuned intracellular thyroid hormone signaling (446). Further studies should elucidate whether stem cell populations in other tissues require the presence of both transporters as well.

Monocarboxylate transporter $\mathbf{1 0}$ Mice that lack MCT10 ubiquitously exhibit increased plasma, muscle, and kidney aromatic amino acids, but no alterations in thyroid hormone homeostasis. However, like Mct8-ko mice, Mct10-ko mice develop inconspicuously and do not exert any obvious neurological defects (23). A physiologically relevant contribution of MCT10 in tissue-specific thyroid hormone transport became evident with the establishment of Mct8/Mct10-dko mice (19). In these animals, thyroid hormone content in the liver, kidney, and thyroid was even further elevated than in Mct8 single-ko animals, suggesting that MCT10 significantly contributes to T3 efflux in these tissues if MCT8 is missing. Most intriguingly, the characteristic abnormal thyroid hormone profile of Mct8-ko mice was partially normalized by the concomitant inactivation of MCT10 because Mct8/Mct10-dko mice exhibit normal serum $\mathrm{T} 4$ values and, as a consequence, a milder brain phenotype than the Mct 8 single-ko animals. Based on these studies in mice, MCT10 may only partially compensate for the absence of MCT8 in tissues in which MCT10 and MCT8 show an overlapping expression pattern (eg, liver, kidney, and thyroid), but not in tissues in which both transporters exhibit a different spatiotemporal expression pattern (eg, CNS). Together with the differences in basic transporter properties of both proteins (see "Substrate Specificity and Transport Direction"), these aspects explain why MCT10 and MCT8 cannot compensate each other. 
The cochlea represents another organ in which a collaborative action of MCT8 and MCT10 was noted. Only mice deficient in MCT8 and MCT10 exhibit deafness and loss of cochlear hair cells, which was accompanied by sensory epithelium degeneration (419). Loss of auditory function could be prevented by exogenous T3 treatment of Mct8/Mct10-dko mice between P7 and P28. T3 treatment also preserved sensory epithelium integrity and hair cell survival, indicating that insufficient T3 signaling during a critical time period is the underlying cause of deafness (419). However, which cellular thyroid hormone transport processes are most effected by combined MCT8/MCT10 deficiency in the hearing system remains enigmatic because both transporters show a rather distinct, nonoverlapping expression (447).

In contrast to the hearing system, coexpression of MCT8 and MCT10 occurs in mouse chondrocytes and skeletal muscle $(24,221)$. Yet, no data are currently available as to whether functional integrity of bone and muscle is disturbed on combined inactivation of both transporters.

Apart from MCT8, MCT10 can act in concert with other transport systems, notably the LATs. In mice, MCT10 and LAT2 seem to cooperate in the renal reabsorption of neutral amino acids. Compared with mice lacking only MCT10 or LAT2, Mct10/Lat2-dko mice lost larger amounts of aromatic and other neutral amino acids because of a tubular reabsorption defect (448). To what extent both transporters also cofacilitate renal thyroid hormone uptake and/or efflux has not been addressed so far. Of note, MCT10 and LAT2 are also coexpressed in cells related to the immune system, such as microglial cells and dendritic cells (449). Moreover, in dendritic cells, T3 was shown to induce interleukin-12 secretion and this response was blunted on pharmacological blocking of MCT10, suggesting that Mct10 mediates T3 transport in this cell type (449).

L-type amino acid transporters Though several members of the LAT family were shown to transport iodothyronine in vitro, most important LAT1 and LAT2, mouse mutants have largely failed to provide solid evidence for such a function in vivo thus far. The phenotype of the respective global ko mouse can be dominated by an impaired amino acid transport that can lead to malnutrition and premature death as observed in LAT4- (SLC43A2-) deficient animals (450). Homozygous inactivation of the catalytic subunit LAT1 (SLC7A5) in mice causes embryonic lethality, whereas heterozygous
Lat1 animals are viable, fertile, and phenotypically similar to wild-type littermates (451). Cellspecific deletion of LAT1 in skeletal muscle cells is accompanied by subtle changes in amino acid transport and intramuscular nutrient signaling (451). However, no data are currently available as to whether heterozygous or muscle-specific Lat 1 mutant mice display an altered thyroid hormone homeostasis. Likewise, mice with inactive LAT1 in endothelial cells display decreased concentrations of leucine and isoleucine in the CNS, supporting the role of LAT1 as a critical transport system for branched-chain amino acids at the BBB (412). These mouse mutants display locomotor dysfunction and autism-related phenotypes, including reduced social interactions. Most important, several patients diagnosed with autism spectrum disorder and motor problems were shown to harbor inactivating mutations in LAT1 (412). Though the neurobehavioral phenotypes of BBB-specific Lat 1 mouse mutants and the symptoms of LAT1 patients may be largely explained by reduced concentrations of branched-chain amino acids in the brain, to what extent LAT1 contributes to passage of thyroid hormones into and within the mouse and human CNS still needs to be unraveled.

In contrast to the global Lat1-ko animals, global Lat2-ko mice are viable and fertile. Determination of thyroid hormone parameters in serum, brain, liver, and kidney revealed normal values (25) or slightly decreased concentrations (26). A moderate neutral aminoaciduria indicates a significant contribution of LAT2 in renal amino acid transport (25). Moreover, Lat2-ko mice display slight locomotor deficits that may be explained by balance impairments due to vestibular damage. Indeed, absence of LAT2 compromises cochlear function by causing an age-dependent loss of spiral ganglia cells and spiral ligament fibrocytes (421). Interestingly, mutations in LAT2 were found in patients with age-related hearing impairment, suggesting a similar function of this transporter in the hearing system of mice and humans (421). Again, it remains to be determined whether a disturbed local thyroid hormone homeostasis represents a major pathogenic mechanism underlying this phenotype.

Owing to the overlapping neuronal expression of Mct8 and Lat2 in the mouse CNS, Mct8/Lat2dko mice were expected to exhibit a pronounced neuronal thyroid hormone deprivation. However, as stated previously, Mct8/Lat2-dko mice fully replicate the phenotype of Mct8-ko mice and display only a mild reduction in thyroid hormone concentrations in the CNS. Only perinatally, could a different thyroid hormone brain status be detected. Whereas Mct8-ko mice exhibit increased 
serum T4 concentrations, brain T4 content, and T3 signaling compared to wild-type mice around birth $(416,417)$, a concomitant deletion of Lat2 normalized the transient brain hyperthyroidism of Mct8-ko mice (26). Though the underlying pathogenic mechanisms are still unclear, this observation suggests an involvement of LAT2 in brain thyroid hormone transport as well.

\section{Therapeutic Approaches in Monocarboxylate Transporter 8 Deficiency}

\section{General considerations}

Effective therapy for MCT8 deficiency entails 1) restoring thyroid hormone signaling in the brain and 2) alleviating the thyrotoxic state in peripheral tissues. It is unclear at what stage brain development is dysregulated in case of MCT8 deficiency, and when therapeutic interventions thus should be initiated to increase thyroid hormone action in the brain. MCT8 is already highly expressed during early fetal brain development that depends on adequate thyroid hormone concentrations (4, 452-454). On these grounds, it seems plausible that initiation of therapeutic interventions prenatally would yield the highest benefit. Yet, most newborns with MCT8 deficiency reportedly have no obvious abnormalities, suggesting that perhaps early postnatal initiation may also be beneficial.

Over the last decade, several therapeutic approaches have been investigated, including classical (anti-)thyroid drugs, thyroid hormone analogues, gene therapy, and chemical chaperones (Fig. 9A-9D) (reviewed in $(255,455,456)$ ). The effects of therapeutic approaches that have been applied in patients with MCT8 deficiency are summarized in Table 4.

\section{Classic (anti-)thyroid drugs}

Based on the low serum FT4 concentrations and modestly increased TSH concentrations, many patients have been suspected to have mild (central) hypothyroidism, and at least 25 of them have been commenced on L-T4 $(14,15,261,322,323$, $325,337,340,344,353,359,360,363,375,378$, $379,384-387,457)$. The ages at start of treatment ranged from 1 month to 3 years. The L-T4 dose ranged from 2.5 to $15 \mathrm{mg} / \mathrm{kg} / \mathrm{d}$, although in the majority of cases no specific dosage has been mentioned. Based on the observations described in these individual cases, L-T4 treatment is deemed to have no beneficial effects on neurocognitive development. Rather, in at least 4 cases serum T3 concentrations were clearly further increased by
L-T4 administration, which is likely to aggravate the hyperthyroid state in peripheral tissues.

Five patients have been reportedly treated with antithyroid drugs, alone or in combination with L-T4 (341, 349, 357, 376, 458). Administration of methimazol had no effects on the serum thyroid function tests (357). Alternatively, the administration of propylthiouracil (PTU), which not only blocks thyroid hormone production but also inhibits the T4 to $\mathrm{T} 3$ conversion, combined with L-T4 has shown some clinical benefits in some patients (341, 357, 376). After 10 to 24 weeks of treatment with PTU at a dose of 200 to $400 \mathrm{mg} / \mathrm{d}$, (F)T3 concentrations normalized, (F)T4 concentrations decreased, and TSH concentrations increased. When L-T4 (typically $100 \mu \mathrm{g} / \mathrm{d}$ ) was added, serum thyroid function tests largely normalized and the thyrotoxic state in peripheral tissues was alleviated, as evidenced by improvement in body weight, heart rate, and serum markers of tissue thyroid hormone state $(341,357)$. No beneficial effect was observed on mental or motor function, although treatment had been initiated between ages 17 and 20 months in 3 of 5 patients (376). Importantly, the treatment with PTU was stopped in one patient because of the occurrence of hypogranulocytosis (376). Owing to this and other potential side effects of PTU, including hepatotoxicity, the use of PTU for treatment of hyperthyroidism is discouraged, particularly in children (459).

\section{Thyroid hormone analogues}

The lack of beneficial effects of L-T4 treatment on neurodevelopment is thought to be attributable to the impaired MCT8-mediated transport of thyroid hormone across the $\mathrm{BBB}$ or into thyroid hormone target cells (Fig. 9A). This has led some groups to investigate the application of thyroid hormone analogues. Such analogues should enter the target cells independent from MCT8, but once inside the cell exert similar effects as T3 (Fig. 9B). The general hypothesis underlying the potential therapeutic mechanism of these analogues consists of the simultaneous inhibition of TSH secretion and hence endogenous thyroid hormone production while providing adequate thyromimetic effects in all body tissues, including the brain (455).

The first thyroid hormone analogue studied in the context of MCT8 deficiency was 3,5-diiodothyropropionic acid (DITPA) by the Refetoff group (460). Compared to T3, DITPA lacks the amine group of the alanine side chain as well as the 3'-iodine moiety. DITPA is a ligand for $\mathrm{TR} \alpha$ and $\operatorname{TR} \beta$, although its affinity is 300-times lower compared to T3, and hence high dosages are required to achieve effects similar to 
(a)

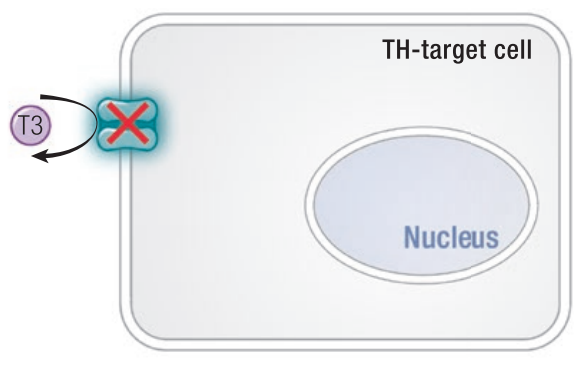

(c)

(c) AAV9-мСт8 (2.

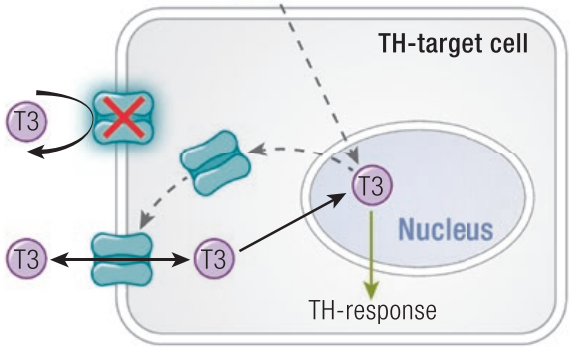

(b)

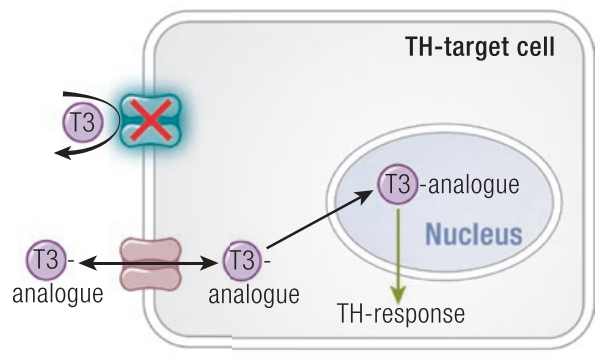

(d)

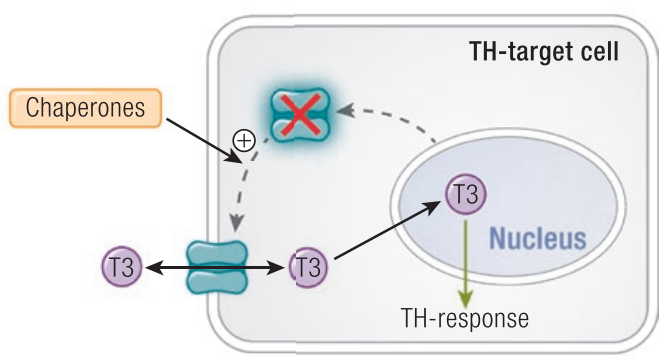

Functional MCT8

Mutant MCT8

Alternative transporter

Figure 9. Overview of therapeutic approaches for MCT8 deficiency that are currently under investigation. A, Schematic representation of an MCT8-dependent thyroid hormone (TH) target cell in which thyroid hormone uptake (including exogenously administrated L-T4) is diminished. B, T3-analogue therapy involves molecules that bypass MCT8 for their cellular entry, but once inside the cell bind to the nuclear TH receptor. Examples are Triac and DITPA. C, Gene therapy aims to supply MCT8-deficient cells with wild-type MCT8 protein through viral vectors that contain the wild-type МСT8 coding sequence. Through selection of specific viral vectors and promoter sequences, specific cell types can be targeted. D, Treatment with chemical (or molecular) chaperones aims to potentiate surface translocation of mutant MCT8 proteins that retained intrinsic transport activity (type 2 mutations). Chaperone treatment may enhance cell surface expression levels.

L-T4 (461). Administration of DITPA to Mct8-ko and control mice resulted in similar tissue availability of DITPA in the liver and brain, suggesting that DITPA enters these tissues independently from MCT8 (460). DITPA lowered serum TSH concentrations effectively in Mct8-ko and wildtype control mice, which was followed by a reduction in serum T4, T3, and rT3 concentrations (460, 462). Consequently, DITPA was found to, at least partially, restore expression levels of several thyroid hormone target genes and deiodinase activity in the liver and brain of Mct8-ko mice (460). Especially the reduction in DIO1 and DIO2 activity following DITPA administration may significantly contribute to the reduction of elevated serum T3 concentrations. Although administration of a low-dose DITPA $(0.3 \mathrm{mg} / 100 \mathrm{~g}$ body weight/d) effectively restored the hypermetabolic state and abnormal serum thyroid function tests in Mct8-ko mice, it had only minor effects on thyroid hormone markers in the brain (462). Obviously the interpretation of these studies is complicated by the lack of neurological derangements in this mouse model. DITPA is able to cross the placenta and regulate thyroid hormone-dependent genes in the fetal mouse cerebral cortex, which would offer the possibility of early treatment initiation (463). DITPA treatment recovered the expression of the myelin marker $p 0$, but only partially rescued hypomyelination in $m c t 8-/-$ zebrafish embryos once administrated early in development $(30,216)$. In line with these preclinical data, treatment of 4 patients with MCT8 deficiency with increasing concentrations of DITPA (final dose $\sim 2.1-2.4 \mathrm{mg} / \mathrm{kg} / \mathrm{d}$ in 3 divided doses), initiated at ages 8.5 to 25 months, normalized the high serum T3 concentrations in all 4 patients and improved several markers of peripheral thyroid hormone action, including serum SHBG concentrations (216). Heart rate and body weight were unchanged in 2 patients (376). However, no improvements were observed in neurodevelopment. 
Table 4. Overview of Treatment Effects in Monocarboxylate Transporter 8-Deficient Patients.

\begin{tabular}{|c|c|c|c|c|}
\hline & L-T4 & L-T4 + PTU & DITPA $_{a}$ & TRIAC \\
\hline Type of Study & Case Reports or Series & Case Reports & Case Series & Phase 2 Clinical Trial \\
\hline References & $\begin{array}{l}(14,15,261,322,323,325,337,340 \\
344,353,359,360,363,375,378 \\
379,384-387,457)\end{array}$ & $(341,349,357,376,458)$ & (376) & (397) \\
\hline No. & 25 & 5 & 4 & 46 \\
\hline Dose ranges & 2.5 to $15 \mu \mathrm{g} / \mathrm{kg} / \mathrm{d}(\sim 25$ to $150 \mu \mathrm{g} / \mathrm{d})$ & $\begin{array}{l}2.5 \text { to } 10 \mu \mathrm{g} / \mathrm{kg} / \mathrm{d} \text { ( } 25 \text { to } \\
100 \mu \mathrm{g} / \mathrm{d})+ \\
200 \text { to } 400 \mathrm{mg} / \mathrm{d}\end{array}$ & $1 \sim 2 \mathrm{mg} / \mathrm{kg} / \mathrm{d}$ & 13 to $88 \mu \mathrm{g} / \mathrm{kg} / \mathrm{d}$ \\
\hline Age at start (range), mo & 20 (0.5 to 36$)$ & 20 (19 to 456$)$ & 25 (8.5 to 25$)$ & 85 (10 to 800$)$ \\
\hline Duration (range), mo & Variable (often n.d.) & $\sim 8.5(1$ to 16$)$ & $\sim 37(26$ to 40$)$ & 13 (0.5 to 42) \\
\hline \multicolumn{5}{|l|}{ Effects } \\
\hline T3 & $=/ \uparrow$ & $\downarrow$ & $\downarrow$ & $\downarrow$ \\
\hline (F)T4 & $=/ \uparrow$ & $\uparrow$ & $\uparrow$ & $\downarrow$ \\
\hline TSH & $\downarrow$ & $\downarrow$ & $=$ & $\downarrow$ \\
\hline SHBG & NA & $\downarrow$ & $\downarrow$ & $\downarrow$ \\
\hline Body weight & $\downarrow$ & $\uparrow$ & $\sim$ & $\uparrow$ \\
\hline Heart rate & NA & $\downarrow$ & $\downarrow$ & $\downarrow$ \\
\hline Development & $=$ & $=$ & $=$ & $\sim /=$ \\
\hline Drug-related adverse outcomes & Increase in peripheral thyrotoxicity & Hypogranulocytosis & Not observed & $\begin{array}{l}\text { Transient mild signs of } \\
\text { hyperthyroidism during } \\
\text { initiation of treatment }\end{array}$ \\
\hline Recommendation & $\begin{array}{l}\text { Avoid: } \\
\text { Not effective } \\
\text { Side effects }\end{array}$ & $\begin{array}{l}\text { Avoid: } \\
\text { Side effects }\end{array}$ & Consider & Consider \\
\hline
\end{tabular}

Abbreviations: $\uparrow$, increase; $\downarrow$, decrease; =, unchanged; , change in subset of patients; DITPA, 3,5-diiodothyropropionic acid; L-T4, levothyroxine; MCT8, monocarboxylate transporter 8; NA, not available; PTU, propylthiouracil; SHBG, sex hormone-binding globulin; Triac, triiodothyroacetic acid; TSH, thyrotropin.

Treatment effects have been scored as follows: for case reports and series the direction of the response observed in the majority ( $>50 \%$ ) of cases with available data is provided; for clinical trials the direction of the response at group level is provided (where $=$ is used for responses that were not statistically significant at group level).

aDITPA is not available for purchase.

Another T3 analogue that has been studied is the naturally occurring thyroid hormone metabolite Triac (464). Triac binds TRa with similar affinity as T3, while binding TR $\beta 2$ times more avidly, suggesting that Triac preferentially acts through TR $\beta$ (465-467). The cellular uptake of Triac is MCT8 independent (192). In vitro, Triac is effectively metabolized by DIO1 and DIO3 and can mimic the effects of $\mathrm{T} 33$ on the regulation of T3-responsive neuronal genes (192). Importantly, once-daily administration of Triac between postnatal day 1 and 12 (200-400 ng/g body weight/d) largely prevented abnormal brain development in the athyroid Pax8-ko mice and Mct8/ Oatp1c1-dko mice (192), evidenced by normal cerebellar Purkinje cell dendritogenesis as well as cortical myelination, which are all known to be thyroid hormone-dependent processes (192).
Administration of its precursor Tetrac, which has a longer half-life than Triac, exhibited similar effects on brain development in Pax8-ko and Pax8/Mct8-dko mice (193). Triac and Tetrac also ameliorated neurodevelopmental abnormalities in zebrafish models for MCT8 deficiency $(30,31,110$, 216) and improved cerebellar Purkinje cell development in the Mct8-deficient chicken model (32). In addition to these neurodevelopmental effects, low doses of Triac applied via the drinking water to juvenile Mct8-ko mice lowered circulating T3 concentrations, suggesting that Triac has the potential to alleviate the peripheral phenotype in MCT8 deficiency (468). As a consequence of the reduced serum thyroid hormone concentrations, brain thyroid hormone content decreased and the expression of some T3-target genes was found to be reduced under these conditions. However, it 
should be noted that Mct8-ko mice have no obvious neurodevelopmental phenotype, and therefore may not be representative of human MCT8 deficiency in the brain (469).

Based on these promising preclinical studies, the first clinical trial was launched in 2014 primarily evaluating the potency of Triac to alleviate thyrotoxicosis in peripheral tissues (33). In this international, multicenter, open-label, single-arm, phase 2 trial, 46 patients with MCT8 deficiency, aged 0.8 to 66 years at time of inclusion, were enrolled and were treated with Triac according to a predefined dose-escalation protocol for a period of 12 months. According to this protocol the Triac dose was increased until serum T3 concentrations were within the target range of 1.4 to $2.5 \mathrm{nmol} / \mathrm{L}$. Forty patients finished the 12 months of treatment, of whom 10 entered an open-label treatment extension period (median follow-up time, 40.4 months). A median Triac daily dose of $37 \mu \mathrm{g} /$ $\mathrm{kg}$ body weight/d effectively lowered serum T3 concentrations. Triac treatment was associated with improvements in heart rate and blood pressure, and the occurrence of premature atrial complexes largely subsided in the majority of patients. Moreover, on Triac treatment, body weight to age $\mathrm{z}$ scores increased and clearly deviated from the natural history in those individuals enrolled in the treatment extension period. In addition, serum markers for tissue thyroid hormone state including SHBG concentrations improved (33). Together, these observations indicate that several key features related to the peripheral phenotype of MCT8 deficiency are alleviated under Triac treatment in pediatric and adult patients. Explorative analysis showed an improvement in motor function in patients who commenced Triac before age 4 years. A second phase 2 trial (NTC02396459) will assess the effects of Triac on neurodevelopmental outcomes in very young infants. Despite its promising effects in preclinical studies and its reported longer plasma halflife, the clinical application of the precursor Tetrac in MCT8 deficiency has not been pursued thus far, mainly because of the very limited clinical experience and absence of marketing-authorized formulations of this drug.

In an attempt to further enhance drug delivery and optimize thyromimetic action in the brain, other thyroid hormone analogues are also currently being studied. These compounds include the synthetic thyromimetic sobetirome and its prodrug Sob-AM2 $(470,471)$. Both compounds accumulate in the brain of Mct8/Dio2-dko mice, with Sob-AM2 yielding higher final sobetirome concentrations in the brain. In the cerebral cortex of these animals, both compounds restored the expression levels of some, predominantly neuronal, thyroid hormone target genes, whereas thyroid hormone target genes in astrocytes and oligodendrocytes showed only minor changes (470). Although both compounds lowered the strongly elevated serum T3 concentrations in Mct8/Dio2-dko mice, the expression levels of several thyroid hormone target genes in heart and liver did not revert to levels observed in wild-type animals (470). Future studies should investigate the effects of these and other thyromimetic drugs in additional animal models of human MCT8 deficiency and compare their effects and safety profile to the clinically available thyroid hormone analogue Triac. In contrast to Triac, DITPA is no longer clinically available.

\section{Gene therapy}

Because MCT8 deficiency is a monogenetic disorder, gene therapy theoretically poses an attractive treatment to restore MCT8 function in tissues where it is of most physiological relevance (Fig. 9C). The first pilot studies in Mct8-ko mice indicated that intravenously administrated AAV9-MCT8 increased the expression of functional MCT8 at the $\mathrm{BBB}$ and brain $\mathrm{T} 3$ concentrations (34). It is unclear, however, whether the moderate increase in MCT8 expression is sufficient to adequately restore thyroid hormone signaling in the brain.

It also remains to be investigated whether reexpression of MCT8 at the $\mathrm{BBB}$ is sufficient to restore thyroid hormone action in the CNS because, at least during development, MCT8 is expressed in a broad variety of other cell types in the brain, including neurons and glial cells. In this context, it would be relevant to study the effects of gene therapy in other (mouse) models for human MCT8 deficiency because the Mct8-ko mice used in the only available study do not exhibit clear brain abnormalities. As outlined in "Expression and Tissue Distribution of Monocarboxylate Transporter 8 and Monocarboxylate Transporter 10," expression of MCT8 in different tissues follows distinct spatiotemporal patterns. Therefore, expression of MCT8 under control of a constitutively active promoter may pose a risk of exposing cells during certain developmental stages to excessive amounts of thyroid hormone, the consequences of which are unknown. Ideally, such vectors should contain the native MCT8 promoter to mimic the dynamics of MCT8 expression as closely as possible. Further studies are thus warranted to optimize the gene delivery strategy and assess its safety. Should gene therapy be effective and safe, it would have obvious practical advantages over life-long medicinal treatments.

Other types of gene therapy, such as gene editing by CRISPR-Cas methodologies, are 
currently not pursued as a treatment for MCT8 deficiency. In particular the widespread distribution of MCT8 in the brain poses a limitation for this approach. Yet, this technique has already proven to be a valuable tool in fundamental research (eg, (28)).

\section{Chemical chaperones}

For a subset of (type 2) mutations, the application of chemical or pharmacological chaperones could restore MCT8 function. This approach is based on the concept that certain mutations result in misfolding of the MCT8 protein, which is in turn rapidly degraded and prevented from reaching the plasma membrane. Chaperones may improve the folding of such mutant proteins and potentiate their trafficking to the plasma membrane and hence (partially) restore their function (Fig. 9D). Several chemical chaperones have been successfully applied in preclinical studies of several genetic disorders, including cystic fibrosis (472). The exact molecular mechanism underlying the positive effects of chaperones is currently unclear, but likely involves modulating the protein quality control system. Depending on the type of chaperone, additional drug targets have been reported $(473,474)$. Several studies by Braun and Schweizer in stably transfected MDCK-1 cells suggest that the function of certain mutant MCT8 proteins can be enhanced by treatment with phenylbutyrate (PBA) or dimethylsulfoxide, and to a lesser extent with genistein $(35,36)$. Those mutations that have been associated with a relatively less severe clinical phenotype, such as the p.F501del mutation, especially appear to respond to this intervention in vitro. However, such beneficial effects were not observed in ex vivo studies in fibroblasts derived from a patient with this p.F501del mutation (37). Further studies are required to assess the effectiveness of chaperones in other models of human MCT8 deficiency, including iPSC-derived vascular endothelial cells and neurons, as well as in vivo models for MCT8 deficiency. The latter would require the knock-in of a mutant Mct8 gene or introduction of targeted mutations by CRISPR-Cas approaches, both of which have not been established yet. Successful in vivo application of chaperones will moreover largely rely on their tissue penetration and the drug tolerability. Because chaperones do not specifically target defective MCT8, treatment with such agents would bear significant risk for off-target effects. In particular in case of PBA, the negative impact of PBA on the body nitrogen pool should be considered once applied to children with poor nutritional status.

\section{Supportive care}

Because therapeutic interventions that effectively restore euthyroidism in all tissues are currently lacking, symptomatic treatment is often necessitated to treat key clinical features such as dystonia and spasticity and seizures. Moreover, a substantial number of patients reportedly required tube feeding to safeguard sufficient dietary intake and/or prevent aspiration. However, many studies did not report in detail on the use of comedication and the occurrence of comorbidities, which hampers proper identification of common medical concerns in MCT8 deficiency and the assessment of the effectiveness of symptomatic interventions.

\section{Future Perspectives}

The number of transporter proteins found to transport thyroid hormones or metabolites thereof is continuously growing, with about 16 human transporters identified to date. One of the challenges remains to elucidate the contribution of each individual transporter to the complex regulation of tissue thyroid hormone homeostasis. Some thyroid hormone transporters also accept a wide range of alternative substrates whose concentrations in plasma or urine are much closer to the apparent transporter $\mathrm{Km}$ values than is the case for thyroid hormones, which have free serum concentrations only in the picomolar range. Although the presence of a multitude of thyroid hormone transporters may provide a sophisticated system to secure adequate thyroid hormone supply in every tissue, some transporters may be more relevant than others. This is illustrated by the devastating effects on brain development of defects in MCT8 $(14,15)$, which is considered the most crucial thyroid hormone transporter. Yet, the recent identification of a patient with apparent OATP1C1 deficiency illustrates that defects in transporters other than MCT8 may also result in novel syndromes of RTH with tissue-specific alterations in thyroid hormone state (16). The increasing availability of nextgeneration sequencing in clinical practice and the establishment of global data repositories will help identify individuals with (pathogenic) variants in thyroid hormone transporters and establish their clinical and biochemical effects.

At the same time, efforts should be made to improve knowledge of known disorders associated with thyroid hormone-transporter defects. For MCT8 deficiency, most of the information available to date relies on individual case descriptions and case series of related patients and often focuses 
on different aspects of the disorder (summarized in, eg, (456)). It is also imperative to systematically describe the various dimensions of the phenotype associated with defective MCT8 and detail their progress over time. Such a natural history is crucial to recognize common features of the disorder that allows optimization of patient care, and to establish a historical control cohort that can be used in the efficacy and safety assessments of any potential novel therapies that reach the clinical stage. The latter is likely necessitated given the severity and rarity of the disorder precluding the conducting of randomized, controlled trials on ethical and logistic grounds. For OATP1C1 deficiency, identification of additional patients will be of great value to better establish the features of this novel disorder and gain insights into its exact pathophysiology. To this end, the establishment of suitable animal models that mimic human features as well as cell-based models will be needed to disentangle the underlying molecular alterations.

It is beyond doubt that establishing an effective therapy for MCT8 deficiency will be one of the major tasks of the field. Research in recent years has predominantly focused on the use of thyroid hormone analogues, of which DITPA and in particular Triac have shown promising results in preclinical studies $(192,413,460)$. A recent clinical trial showed that Triac effectively ameliorates the peripheral phenotype of MCT8 deficiency (33), which has long been an unmet medical need for patients with this disorder. Further clinical studies will reveal whether and to what extent Triac can positively modulate neurodevelopment. Additional lines of ongoing research focus on alternative approaches to restore or circumvent defective MCT8, including gene therapy and application of other thyroid hormone analogues or chaperone molecules in selected patients.

Another major remaining challenge is to elucidate the contribution of individual thyroid hormone transporters to cellular thyroid hormone homeostasis under physiological and pathophysiological conditions. Although the profound phenotypes of patients with specific transporter defects have provided an answer to some questions, many others remain to be addressed through studies in animals and advanced cellular models. It should be acknowledged that global ko animals may not always pose the most optimal model to study the respective thyroid hormone transporter function in selected organs because it may be masked by systemic effects. Even within the same tissue, different cell populations may require different transporters to ascertain adequate thyroid hormone signaling. Consequently, generating and studying cell-specific transporter ko mice appears to be an attractive approach that is expected to disclose further distinct thyroid hormone transporter functions.

For a long time, a major gap in the field had been the absence of crystal structures of thyroid hormone transporters or any homologous proteins. Although structural homology models have broadened our understanding of the mechanism by which thyroid hormone transporters translocate the various iodothyronine substrates (eg, $(77,153,252,253,276)$, the sequence similarity to template structures is generally low, preventing accurate molecular predictions. In this context, thorough functional characterization of the WT and mutant thyroid hormone transporters will be a prerequisite to interpret the impact of any genetic variant and prove pathogenicity in case of MCT8 mutations. The recent elucidation of the LAT1 protein structure will undoubtedly provide more detailed insights into the mechanism by which it transports iodothyronines (268). Because LAT1 has a protein fold different from the thyroid hormone transporters that belong to other protein families, it most likely cannot serve as a template to model the structure of other thyroid hormone transporters. Resolving the molecular structure of additional thyroid hormone transporters or homologous proteins, will advance the application of in silico approaches in substrate discovery, the design of transporterspecific inhibitors, and the prediction the pathogenicity of amino acid substitutions.

So far, most research has focused on the transport of the classic thyroid hormones T3 and T4, with some studying 3,3'-T2 and rT3. However, compiling evidence suggests that also other metabolites are being formed in vivo that likely also require transporter proteins to cross the plasma membrane. Such transporters remain to be identified. Moreover, the renal and hepatic transport of sulfoconjugated and glucuronideconjugated iodothyronines that represent major thyroid hormone metabolites in bile and urine is still poorly understood. In a field in which studies have long been dependent on the availability of $\left[{ }^{125} \mathrm{I}\right]$-radio-labeled iodothyronines $(475,476)$, the establishment of nonradioactive assays based on Sandell-Kolthoff reactions $(477,478)$ or liquid chromatography-mass spectrometry techniques (77, 479) for determining iodothyronine concentrations have already significantly improved 
thyroid hormone research and are expected to greatly amplify the number of potential transporter substrates that can be studied.

Although major advancements have been made in the field of thyroid hormone transport during the last 15 years, many unknowns still need to be resolved. With the recent identification of a novel thyroid hormone transporter and a second disorder associated with a thyroid hormone-transporter defect, as well as the implementation of more sophisticated models and experimental techniques, the field is ready to take the next step forward in unraveling the complex mechanisms involved in the regulation of tissue thyroid hormone state.

\section{References}

1. Mullur R, Liu YY, Brent GA. Thyroid hormone regulation of metabolism. Physiol Rev. 2014;94(2):355-382.

2. Yen PM. Physiological and molecular basis of thyroid hormone action. Physiol Rev. 2001;81(3):1097-1142

3. Gereben B, Zavacki AM, Ribich S, et al. Cellular and molecular basis of deiodinase-regulated thyroid hormone signaling. Endocr Rev. 2008;29(7):898-938.

4. Bernal J, Guadaño-Ferraz A, Morte B. Thyroid hormone transporters-functions and clinical implications. Nat Rev Endocrinol. 2015;11(7):406-417.

5. Bianco AC, Dumitrescu A, Gereben B, et al. Paradigms of dynamic control of thyroid hormone signaling. Endocr Rev. 2019;40(4):1000-1047.

6. Pappa T, Ferrara AM, Refetoff S. Inherited defects of thyroxine-binding proteins. Best Pract Res Clin Endocrinol Metab. 2015;29(5):735-747.

7. Davis PJ, Goglia F, Leonard JL. Nongenomic actions of thyroid hormone. Nat Rev Endocrinol. 2016;12(2):111-121.

8. Hennemann $G$, Docter R, Friesema EC, de Jong $M$, Krenning EP, Visser TJ. Plasma membrane transport of thyroid hormones and its role in thyroid hormone metabolism and bioavailability. Endocr Rev. 2001;22(4):451-476.

9. Krenning EP, Docter R, Bernard HF, Visser TJ, Hennemann G. Active transport of triiodothyronine (T3) into isolated rat liver cells. FEBS Lett. 1978;91(1):113-116.

10. Friesema EC, Ganguly S, Abdalla A, Manning Fox JE, Halestrap AP, Visser TJ. Identification of monocarboxylate transporter 8 as a specific thyroid hormone transporter. J Biol Chem. 2003;278(41):40128-40135.

11. Friesema EC, Jansen J, Jachtenberg JW, Visser WE, Kester MH, Visser TJ. Effective cellular uptake and efflux of thyroid hormone by human monocarboxylate transporter 10. Mol Endocrinol. 2008;22(6):1357-1369.

12. Teumer A, Chaker L, Groeneweg S, et al; Lifelines Cohort Study. Genome-wide analyses identify a role for SLC17A4 and AADAT in thyroid hormone regulation. Nat Commun. 2018;9(1):4455.

13. Pizzagalli F, Hagenbuch B, Stieger B, Klenk U, Folkers G, Meier PJ. Identification of a novel human organic anion transporting polypeptide as a high affinity thyroxine transporter. $\mathrm{Mol}$ Endocrinol. 2002;16(10):2283-2296.

14. Friesema EC, Grueters A, Biebermann $\mathrm{H}$, et al. Association between mutations in a thyroid hormone transporter and severe $X$-linked psychomotor retardation. Lancet. 2004;364(9443):1435-1437.
15. Dumitrescu AM, Liao XH, Best TB, Brockmann K, Refetoff S. A novel syndrome combining thyroid and neurological abnormalities is associated with mutations in a monocarboxylate transporter gene. Am J Hum Genet. 2004;74(1):168-175.

16. Strømme P, Groeneweg S, Lima de Souza EC, et al. Mutated thyroid hormone transporter OATP1C1 associates with severe brain hypometabolism and juvenile neurodegeneration. Thyroid. 2018;28(11):1406-1415.

17. Mayerl S, Müller J, Bauer R, et al. Transporters MCT8 and OATP1C1 maintain murine brain thyroid hormone homeostasis. J Clin Invest. 2014;124(5):1987-1999.

18. Mayerl S, Visser TJ, Darras VM, Horn S, Heuer $\mathrm{H}$. Impact of Oatp1c1 deficiency on thyroid hormone metabolism and action in the mouse brain. Endocrinology. 2012;153(3):1528-1537.

19. Müller J, Mayerl S, Visser TJ, et al. Tissue-specific alterations in thyroid hormone homeostasis in combined Mct10 and Mct8 deficiency. Endocrinology. 2014;155(1):315-325.

20. Trajkovic M, Visser TJ, Mittag J, et al. Abnormal thyroid hormone metabolism in mice lacking the monocarboxylate transporter 8. J Clin Invest. 2007;117(3):627-635.

21. Dumitrescu AM, Liao XH, Weiss RE, Millen K, Refetoff S. Tissue-specific thyroid hormone deprivation and excess in monocarboxylate transporter (mct) 8-deficient mice. Endocrinology. 2006;147(9):4036-4043.

22. Liao $\mathrm{XH}$, Di Cosmo C, Dumitrescu AM, et al. Distinct roles of deiodinases on the phenotype of Mct8 defect: a comparison of eight different mouse genotypes. Endocrinology. 2011;152(3):1180-1191.

23. Mariotta L, Ramadan T, Singer D, et al. T-type amino acid transporter TAT1 (Slc16a10) is essential for extracellular aromatic amino acid homeostasis control. J Physiol. 2012;590(24):6413-6424.

24. Mayerl S, Schmidt $M$, Doycheva D, et al. Thyroid hormone transporters MCT8 and OATP1C1 control skeletal muscle regeneration. Stem Cell Reports. 2018;10(6):1959-1974.

25. Braun D, Wirth EK, Wohlgemuth F, et al. Aminoaciduria, but normal thyroid hormone levels and signalling, in mice lacking the amino acid and thyroid hormone transporter Slc7a8. Biochem J. 2011;439(2):249-255.

26. Núñez B, Martínez de Mena R, Obregon MJ, et al. Cerebral cortex hyperthyroidism of newborn mct8-deficient mice transiently suppressed by lat2 inactivation. PloS One. 2014;9(5):e96915.

27. Stohn JP, Martinez ME, Matoin K, et al. MCT8 deficiency in male mice mitigates the phenotypic abnormalities associated with the absence of a functional type 3 deiodinase. Endocrinology. 2016;157(8):3266-3277.
28. Vatine GD, Al-Ahmad A, Barriga BK, et al. Modeling psychomotor retardation using iPSCs from MCT8-deficient patients indicates a prominent role for the blood-brain barrier. Cell Stem Cell. 2017;20(6):831-843.e5.

29. Vatine GD, Zada D, Lerer-Goldshtein $T$, et al. Zebrafish as a model for monocarboxyl transporter 8-deficiency. J Biol Chem. 2013;288(1):169-180.

30. Zada D, Tovin A, Lerer-Goldshtein T, Appelbaum L. Pharmacological treatment and BBB-targeted genetic therapy for MCT8-dependent hypomyelination in zebrafish. Dis Model Mech. 2016;9(11):1339-1348

31. de Vrieze E, van de Wiel SM, Zethof J, Flik G, Klaren PH, Arjona FJ. Knockdown of monocarboxylate transporter 8 (mct8) disturbs brain development and locomotion in zebrafish. Endocrinology. 2014;155(6):2320-2330.

32. Delbaere J, Vancamp P, Van Herck SL, et al. MCT8 deficiency in Purkinje cells disrupts embryonic chicken cerebellar development. J Endocrinol. 2017;232(2):259-272.

33. Groeneweg $S$, Peeters RP, Moran $C$, et al. Effectiveness and safety of the tri-iodothyronine analogue Triac in children and adults with MCT8 deficiency: an international, single-arm, openlabel, phase 2 trial. Lancet Diabetes Endocrinol. 2019;7(9):695-706.

34. Iwayama $\mathrm{H}$, Liao $\mathrm{XH}$, Braun $\mathrm{L}$, et al. Adeno associated virus 9-based gene therapy delivers a functional monocarboxylate transporter 8, improving thyroid hormone availability to the brain of Mct8deficient mice. Thyroid. 2016;26(9):1311-1319.

35. Braun D, Schweizer U. The chemical chaperone phenylbutyrate rescues MCT8 mutations associated with milder phenotypes in patients with allan-herndon-dudley syndrome. Endocrinology. 2017;158(3):678-691.

36. Braun D, Schweizer U. Efficient activation of pathogenic $\triangle$ Phe501 mutation in monocarboxylate transporter 8 by chemical and pharmacological chaperones. Endocrinology. 2015;156(12):4720-4730.

37. Groeneweg $S$, van den Berge A, Meima ME, Peeters RP, Visser TJ, Visser WE. Effects of chemical chaperones on thyroid hormone transport by MCT8 mutants in patient-derived fibroblasts. Endocrinology. 2018;159(3):1290-1302.

38. Hagenbuch B, Stieger B. The SLCO (former SLC21) superfamily of transporters. Mol Aspects Med. 2013;34(2-3):396-412.

39. Hagenbuch B, Meier PJ. Organic anion transporting polypeptides of the OATP/ SLC21 family: phylogenetic classification as OATP/ SLCO superfamily, new nomenclature and molecular/functional properties. Pflugers Arch. 2004;447(5):653-665.

40. Fujiwara K, Adachi H, Nishio T, et al. Identification of thyroid hormone transporters 
in humans: different molecules are involved in a tissue-specific manner. Endocrinology. 2001;142(5):2005-2012

41. Kullak-Ublick GA, Ismair MG, Stieger B, et al. Organic anion-transporting polypeptide B (OATP-B) and its functional comparison with three other OATPs of human liver. Gastroenterology. 2001;120(2):525-533.

42. van der Deure WM, Peeters RP, Visser TJ. Molecular aspects of thyroid hormone transporters, including MCT8, MCT10, and OATPs, and the effects of genetic variation in these transporters. J Mol Endocrinol. 2010;44(1):1-11.

43. Gao B, Huber RD, Wenzel A, et al. Localization of organic anion transporting polypeptides in the rat and human ciliary body epithelium. Exp Eye Res. 2005;80(1):61-72.

44. Loubière LS, Vasilopoulou E, Glazier JD, et al. Expression and function of thyroid hormone transporters in the microvillous plasma membrane of human term placental syncytiotrophoblast. Endocrinology. 2012;153(12):6126-6135.

45. Lee W, Glaeser H, Smith LH, et al. Polymorphisms in human organic anion-transporting polypeptide 1A2 (OATP1A2): implications for altered drug disposition and central nervous system drug entry. J Biol Chem. 2005;280(10):9610-9617.

46. Bronger $\mathrm{H}$, König J, Kopplow K, et al. ABCC drug efflux pumps and organic anion uptake transporters in human gliomas and the blood-tumor barrier. Cancer Res. 2005;65(24):11419-11428.

47. König J, Cui Y, Nies AT, Keppler D. A novel human organic anion transporting polypeptide localized to the basolateral hepatocyte membrane. Am J Physiol Gastrointest Liver Physiol. 2000;278(1):G156-G164.

48. Abe T, Kakyo M, Tokui T, et al. Identification of a novel gene family encoding human liver-specific organic anion transporter LST-1. J Biol Chem. 1999;274(24):17159-17163.

49. van der Deure WM, Friesema EC, de Jong FJ, et al. Organic anion transporter 1B1: an important factor in hepatic thyroid hormone and estrogen transport and metabolism. Endocrinology. 2008;149(9):4695-4701.

50. Roth M, Obaidat A, Hagenbuch B. OATPs, OATs and OCTs: the organic anion and cation transporters of the SLCO and SLC22A gene superfamilies. $\mathrm{Br}$ J Pharmacol. 2012;165(5):1260-1287.

51. Tamai I, Nezu J, Uchino H, et al. Molecular identification and characterization of novel members of the human organic anion transporter (OATP) family. Biochem Biophys Res Commun. 2000;273(1):251-260.

52. Cui Y, König J, Leier I, Buchholz U, Keppler D. Hepatic uptake of bilirubin and its conjugates by the human organic anion transporter SLC21A6. J Biol Chem. 2001;276(13):9626-9630.

53. Ji C, Tschantz WR, Pfeifer ND, Ullah $M$, Sadagopan N. Development of a multiplex UPLC-MRM MS method for quantification of human membrane transport proteins OATP1B1, OATP1B3 and OATP2B1 in in vitro systems and tissues. Anal Chim Acta. 2012;717:67-76.

54. Abe T, Unno M, Onogawa T, et al. LST-2, a human liver-specific organic anion transporter, determines methotrexate sensitivity in gastrointestinal cancers. Gastroenterology. 2001;120(7):1689-1699.

55. Leuthold S, Hagenbuch B, Mohebbi N, Wagner CA, Meier PJ, Stieger B. Mechanisms of $\mathrm{pH}$-gradient driven transport mediated by organic anion polypeptide transporters. Am J Physiol Cell Physiol. 2009;296(3):C570-C582.
56. König J, Cui Y, Nies AT, Keppler D. Localization and genomic organization of a new hepatocellular organic anion transporting polypeptide. J Biol Chem. 2000;275(30):23161-23168.

57. van der Deure WM, Hansen PS, Peeters RP, et al. Thyroid hormone transport and metabolism by organic anion transporter $1 \mathrm{C} 1$ and consequences of genetic variation. Endocrinology. 2008;149(10):5307-5314.

58. López-Espíndola D, García-Aldea Á, Gómez de la Riva I, et al. Thyroid hormone availability in the human fetal brain: novel entry pathways and role of radial glia. Brain Struct Funct. 2019;224(6):2103-2119.

59. Huber RD, Gao B, Sidler Pfändler MA, et al. Characterization of two splice variants of human organic anion transporting polypeptide $3 \mathrm{~A} 1$ isolated from human brain. Am J Physiol Cell Physiol. 2007;292(2):C795-C806.

60. Mikkaichi T, Suzuki T, Onogawa T, et al. Isolation and characterization of a digoxin transporter and its rat homologue expressed in the kidney. Proc Natl Acad Sci U S A. 2004;101(10):3569-3574.

61. Visser WE, Wong WS, van Mullem AA, Friesema EC, Geyer J, Visser TJ. Study of the transport of thyroid hormone by transporters of the SLC10 family. Mol Cell Endocrinol. 2010;315(1-2):138-145.

62. Geyer J, Wilke T, Petzinger E. The solute carrier family SLC10: more than a family of bile acid transporters regarding function and phylogenetic relationships. Naunyn Schmiedebergs Arch Pharmacol. 2006;372(6):413-431.

63. Kersseboom S, van Gucht ALM, van Mullem A, et al. Role of the bile acid transporter SLC10A1 in liver targeting of the lipid-lowering thyroid hormone analog eprotirome. Endocrinology. 2017; 158(10):3307-3318.

64. Haag $M$, Hofmann $U$, Mürdter $T E$, et al. Quantitative bile acid profiling by liquid chromatography quadrupole time-of-flight mass spectrometry: monitoring hepatitis B therapy by a novel $\mathrm{Na}(+)$-taurocholate cotransporting polypeptide inhibitor. Anal Bioanal Chem. 2015;407(22):6815-6825.

65. Togawa N, Miyaji T, Izawa S, Omote $\mathrm{H}$, Moriyama Y. A Na+-phosphate cotransporter homologue (SLC17A4 protein) is an intestinal organic anion exporter. Am J Physiol Cell Physiol. 2012;302(11):C1652-C1660.

66. Mastroberardino L, Spindler B, Pfeiffer R, et al. Amino-acid transport by heterodimers of 4F2hc/ CD98 and members of a permease family. Nature. 1998;395(6699):288-291.

67. Zevenbergen C, Meima ME, Lima de Souza EC, et al. Transport of iodothyronines by human L-type amino acid transporters. Endocrinology. 2015;156(11):4345-4355.

68. Yanagida O, Kanai Y, Chairoungdua A, et al. Human L-type amino acid transporter 1 (LAT1): characterization of function and expression in tumor cell lines. Biochim Biophys Acta. 2001;1514(2):291-302.

69. Takahashi $Y$, Nishimura T, Higuchi $K$, et al. Transport of pregabalin via L-type amino acid transporter 1 (SLC7A5) in human brain capillary endothelial cell line. Pharm Res. 2018;35(12):246.

70. Uchino H, Kanai Y, Kim DK, et al. Transport of amino acid-related compounds mediated by L-type amino acid transporter 1 (LAT1): insights into the mechanisms of substrate recognition. $\mathrm{Mol}$ Pharmacol. 2002;61(4):729-737.

71. Bodoy S, Martín L, Zorzano A, Palacín M, Estévez R, Bertran J. Identification of LAT4, a novel amino acid transporter with system L activity. J Biol Chem. 2005;280(12):12002-12011.

72. Bassi MT, Sperandeo MP, Incerti B, et al. SLC7A8, a gene mapping within the lysinuric protein intolerance critical region, encodes a new member of the glycoprotein-associated amino acid transporter family. Genomics. 1999;62(2):297-303.

73. Pineda $M$, Fernández $E$, Torrents $D$, et al. Identification of a membrane protein, LAT-2, that co-expresses with 4F2 heavy chain, an L-type amino acid transport activity with broad specificity for small and large zwitterionic amino acids. J Biol Chem. 1999;274(28):19738-19744.

74. Babu E, Kanai Y, Chairoungdua A, et al. Identification of a novel system $\mathrm{L}$ amino acid transporter structurally distinct from heterodimeric amino acid transporters. J Biol Chem. 2003;278(44):43838-43845.

75. Cleal JK, Glazier JD, Ntani G, et al. Facilitated transporters mediate net efflux of amino acids to the fetus across the basal membrane of the placental syncytiotrophoblast. J Physiol. 2011;589(Pt 4):987-997.

76. Friesema EC, Kuiper GG, Jansen J, Visser TJ, Kester $\mathrm{MH}$. Thyroid hormone transport by the human monocarboxylate transporter 8 and its rate-limiting role in intracellular metabolism. $\mathrm{Mol}$ Endocrinol. 2006;20(11):2761-2772.

77. Kinne A, Kleinau G, Hoefig CS, et al. Essential molecular determinants for thyroid hormone transport and first structural implications for monocarboxylate transporter 8. J Biol Chem. 2010;285(36):28054-28063.

78. Johannes J, Braun D, Kinne A, Rathmann D, Köhrle J, Schweizer U. Few amino acid exchanges expand the substrate spectrum of monocarboxylate transporter 10. Mol Endocrinol. 2016;30(7):796-808.

79. Johannes J, Jayarama-Naidu R, Meyer F, et al. Silychristin, a flavonolignan derived from the milk thistle, is a potent inhibitor of the thyroid hormone transporter MCT8. Endocrinology. 2016;157(4):1694-1701.

80. Kinne A, Roth S, Biebermann H, Köhrle J, Grüters A, Schweizer U. Surface translocation and tri-iodothyronine uptake of mutant MCT8 proteins are cell type-dependent. J Mol Endocrinol. 2009;43(6):263-271.

81. Kim DK, Kanai $Y$, Matsuo $H$, et al. The human T-type amino acid transporter-1: characterization, gene organization, and chromosomal location. Genomics. 2002;79(1):95-103.

82. Friesema EC, Visser TJ, Borgers AJ, et al. Thyroid hormone transporters and deiodinases in the developing human hypothalamus. Eur J Endocrinol. 2012;167(3):379-386.

83. Segawa $H$, Fukasawa $Y$, Miyamoto $K$, Takeda $E$, Endou $H$, Kanai $Y$. Identification and functional characterization of a $\mathrm{Nat-}$ independent neutral amino acid transporter with broad substrate selectivity. J Biol Chem. 1999;274(28):19745-19751.

84. Jacquemin E, Hagenbuch B, Stieger B, Wolkoff AW, Meier PJ. Expression cloning of a rat liver $\mathrm{Na}(+)$ independent organic anion transporter. Proc Natl Acad Sci U S A. 1994;91(1):133-137.

85. Eckhardt U, Schroeder A, Stieger B, et al. Polyspecific substrate uptake by the hepatic organic anion transporter Oatp1 in stably transfected $\mathrm{CHO}$ cells. Am J Physiol. 1999;276(4):G1037-G1042.

86. Meier PJ, Eckhardt U, Schroeder A, Hagenbuch B, Stieger B. Substrate specificity of sinusoidal bile acid and organic anion uptake systems in rat and human liver. Hepatology. 1997;26(6):1667-1677. 
87. Friesema EC, Docter R, Moerings EP, et al. Identification of thyroid hormone transporters. Biochem Biophys Res Commun 1999:254(2):497-501.

88. Kullak-Ublick GA, Hagenbuch B, Stieger B, et al. Molecular and functional characterization of an organic anion transporting polypeptide cloned from human liver. Gastroenterology. 1995;109(4):1274-1282.

89. Kullak-Ublick GA, Fisch T, Oswald M, et al. Dehydroepiandrosterone sulfate (DHEAS): identification of a carrier protein in human liver and brain. FEBS Lett. 1998;424(3):173-176.

90. König J, Seithel A, Gradhand U, Fromm MF. Pharmacogenomics of human OATP transporters. Naunyn Schmiedebergs Arch Pharmacol. 2006;372(6):432-443.

91. Hagenbuch B. Cellular entry of thyroid hormones by organic anion transporting polypeptides. Best Pract Res Clin Endocrinol Metab. 2007;21(2):209-221.

92. Takeuchi A, Masuda S, Saito H, Abe T, Inui K. Multispecific substrate recognition of kidney-specific organic anion transporters OAT-K1 and OAT-K2. J Pharmacol Exp Ther. 2001;299(1):261-267.

93. Abe T, Kakyo M, Sakagami H, et al. Molecular characterization and tissue distribution of a new organic anion transporter subtype (oatp3) that transports thyroid hormones and taurocholate and comparison with oatp2. I Biol Chem. 1998;273(35):22395-22401.

94. Cattori V, Hagenbuch B, Hagenbuch N, et al. Identification of organic anion transporting polypeptide 4 (Oatp4) as a major full-length isoform of the liver-specific transporter- 1 (rlst- 1 ) in rat liver. FEBS Lett. 2000;474(2-3):242-245.

95. Sugiyama $D$, Kusuhara $H$, Taniguchi $H$, et al. Functional characterization of rat brain-specific organic anion transporter (Oatp14) at the blood-brain barrier: high affinity transporter for thyroxine. I Biol Chem. 2003;278(44):43489-43495.

96. Suzuki T, Onogawa T, Asano N, et al. Identification and characterization of novel rat and human gonad-specific organic anion transporters. Mol Endocrinol. 2003;17(7):1203-1215.

97. Westholm DE, Salo DR, Viken KJ, Rumbley JN, Anderson GW. The blood-brain barrier thyroxine transporter organic anion-transporting polypeptide $1 \mathrm{c} 1$ displays atypical transport kinetics. Endocrinology. 2009;150(11):5153-5162.

98. Hagenbuch B, Gui C. Xenobiotic transporters of the human organic anion transporting polypeptides (OATP) family. Xenobiotica. 2008;38(7-8):778-801.

99. Kobayashi D, Nozawa T, Imai K, Nezu J, Tsuji A, Tamai I. Involvement of human organic anion transporting polypeptide OATP-B (SLC21A9) in $\mathrm{pH}$-dependent transport across intestinal apical membrane. J Pharmacol Exp Ther 2003;306(2):703-708.

100. Shirasaka Y, Shichiri M, Mori T, Nakanishi T, Tamai I. Major active components in grapefruit, orange, and apple juices responsible for OATP2B1-mediated drug interactions. J Pharm Sci. 2013;102(1):280-288.

101. Johnson E), Won CS, Köck K, Paine MF. Prioritizing pharmacokinetic drug interaction precipitants in natural products: application to OATP inhibitors in grapefruit juice. Biopharm Drug Dispos. 2017;38(3):251-259.

102. Rebello $S$, Zhao $S$, Hariry $S$, et al. Intestinal OATP1A2 inhibition as a potential mechanism for the effect of grapefruit juice on aliskiren pharmacokinetics in healthy subjects. Eur J Clin Pharmacol. 2012;68(5):697-708.

103. Satoh H, Yamashita F, Tsujimoto $M$, et al. Citrus juices inhibit the function of human organic anion-transporting polypeptide OATP-B. Drug Metab Dispos. 2005;33(4):518-523.

104. Akanuma S, Hirose S, Tachikawa M, Hosoya K. Localization of organic anion transporting polypeptide (Oatp) $1 \mathrm{a} 4$ and Oatp $1 \mathrm{c} 1$ at the rat bloodretinal barrier. Fluids Barriers CNS. 2013;10(1):29.

105. Sun YN, Liu YJ, Zhang L, et al. Expression of organic anion transporting polypeptide $1 \mathrm{c} 1$ and monocarboxylate transporter 8 in the rat placental barrier and the compensatory response to thyroid dysfunction. PloS One. 2014;9(4):e96047.

106. Grijota-Martínez C, Díez D, Morreale de Escobar G, Bernal J, Morte B. Lack of action of exogenously administered T3 on the fetal rat brain despite expression of the monocarboxylate transporter 8 . Endocrinology. 2011;152(4):1713-1721.

107. Tohyama K, Kusuhara H, Sugiyama Y. Involvement of multispecific organic anion transporter, Oatp14 (Slc21a14), in the transport of thyroxine across the blood-brain barrier. Endocrinology. 2004; 145(9):4384-4391.

108. Roberts LM, Woodford K, Zhou M, et al. Expression of the thyroid hormone transporters monocarboxylate transporter-8 (SLC16A2) and organic ion transporter-14 (SLCO1C1) at the blood-brain barrier. Endocrinology. 2008; 149(12):6251-6261.

109. Ito K, Uchida Y, Ohtsuki S, et al. Quantitative membrane protein expression at the blood-brain barrier of adult and younger cynomolgus monkeys. I Pharm Sci. 2011;100(9):3939-3950.

110. Walter KM, Miller GW, Chen X, Yaghoobi B, Puschner B, Lein PJ. Effects of thyroid hormone disruption on the ontogenetic expression of thyroid hormone signaling genes in developing zebrafish (Danio rerio). Gen Comp Endocrinol. 2019;272:20-32.

111. Wittmann G, Szabon J, MohácsikP, et al. Parallel regulation of thyroid hormone transporters OATP1c1 and MCT8 during and after endotoxemia at the blood-brain barrier of male rodents. Endocrinology. 2015; 156(4):1552-1564.

112. Claro da Silva T, Polli JE, Swaan PW. The solute carrier family 10 (SLC10): beyond bile acid transport. Mol Aspects Med. 2013;34(2-3):252-269.

113. Hagenbuch B, Dawson P. The sodium bile salt cotransport family SLC10. Pflugers Arch. 2004; $447(5): 566-570$.

114. Hagenbuch B, Lübbert H, Stieger B, Meier PJ. Expression of the hepatocyte $\mathrm{Na}+/$ bile acid cotransporter in Xenopus laevis oocytes. J Biol Chem. 1990;265(10):5357-5360.

115. Hagenbuch B, Stieger B, Foguet $M$, Lübbert $H$, Meier PJ. Functional expression cloning and characterization of the hepatocyte $\mathrm{Na}+$ /bile acid cotransport system. Proc Natl Acad Sci U S A. 1991:88(23):10629-10633.

116. Hagenbuch B, Meier PJ. Sinusoidal (basolateral) bile salt uptake systems of hepatocytes. Semin Liver Dis. 1996;16(2):129-136.

117. Trauner M, Boyer JL. Bile salt transporters: molecular characterization, function, and regulation. Physiol Rev. 2003;83(2):633-671.

118. Li W, Urban S. Entry of hepatitis B and hepatitis D virus into hepatocytes: Basic insights and clinical implications.) Hepatol. 2016;64(1 Suppl):S32-S40.

119. Slijepcevic D, Kaufman C, Wichers CG, et al. Impaired uptake of conjugated bile acids and hepatitis $\mathrm{b}$ virus pres 1 -binding in $\mathrm{na}(+)$-taurocholate cotransporting polypeptide knockout mice. Hepatology. 2015;62(1):207-219.

120. Bogomolov P, Alexandrov A, Voronkova N, et al. Treatment of chronic hepatitis $D$ with the entry inhibitor myrcludex B: first results of a phase Ib/lla study. J Hepatol. 2016;65(3):490-498.

121. Ananthanarayanan $\mathrm{M}, \mathrm{Ng}$ OC, Boyer JL, Suchy FJ. Characterization of cloned rat liver $\mathrm{Na}(+)$-bile acid cotransporter using peptide and fusion protein antibodies. Am J Physiol. 1994;267(4 Pt 1):G637-G643.

122. Stieger B, Hagenbuch B, Landmann L, Höchli $M$, Schroeder A, Meier PJ. In situ localization of the hepatocytic $\mathrm{Na}+$ /Taurocholate cotransporting polypeptide in rat liver. Gastroenterology. 1994;107(6): 1781-1787.

123. Boyer JL, Hagenbuch B, Ananthanarayanan $M$, Suchy F, Stieger B, Meier PJ. Phylogenic and ontogenic expression of hepatocellular bile acid transport. Proc Natl Acad SciU S A. 1993;90(2):435-438.

124. Cheng X, Buckley D, Klaassen CD. Regulation of hepatic bile acid transporters Ntcp and Bsep expression. Biochem Pharmacol. 2007;74(11):1665-1676.

125. Kullak-Ublick GA, Glasa J, Böker C, et al. Chlorambucil-taurocholate is transported by bile acid carriers expressed in human hepatocellular carcinomas. Gastroenterology. 1997;113(4):1295-1305.

126. Chen HL, Chen HL, Liu YJ, et al. Developmental expression of canalicular transporter genes in human liver. J Hepatol. 2005;43(3):472-477.

127. Baghdasaryan A, Chiba P, Trauner M. Clinical application of transcriptional activators of bile salt transporters. Mol Aspects Med. 2014;37:57-76.

128. Reimer RJ. SLC17: a functionally diverse family of organic anion transporters. Mol Aspects Med. 2013;34(2-3):350-359.

129. Murer $\mathrm{H}$, Hernando N, Forster I, Biber J. Proximal tubular phosphate reabsorption: molecular mechanisms. Physiol Rev. 2000;80(4):1373-1409.

130. Miyamoto K, Haito-Sugino S, Kuwahara S, et al. Sodium-dependent phosphate cotransporters: lessons from gene knockout and mutation studies. J Pharm Sci. 2011;100(9):3719-3730.

131. Sreedharan S, Shaik JH, Olszewski PK, Levine AS, Schiöth HB, Fredriksson R. Glutamate, aspartate and nucleotide transporters in the SLC17 family form four main phylogenetic clusters: evolution and tissue expression. BMC Genomics. 2010;11:17.

132. Jutabha P, Anzai N, Kitamura K, et al. Human sodium phosphate transporter 4 (hNPT4/SLC17A3) as a common renal secretory pathway for drugs and urate.J Biol Chem. 2010;285(45):35123-35132.

133. Chiba T, Matsuo H, Kawamura Y, et al. NPT1/ SLC17A1 is a renal urate exporter in humans and its common gain-of-function variant decreases the risk of renal underexcretion gout. Arthritis Rheumatol. 2015;67(1):281-287.

134. Shibui A, Tsunoda T, Seki N, Suzuki Y, Sugane K, Sugano S. Isolation and chromosomal mapping of a novel human gene showing homology to $\mathrm{Na}+/ \mathrm{PO} 4$ cotransporter. J Hum Genet. 1999;44(3):190-192

135. Palacín M. A new family of proteins (rBAT and $4 \mathrm{~F} 2 \mathrm{hc}$ ) involved in cationic and zwitterionic amino acid transport: a tale of two proteins in search of a transport function.J Exp Biol. 1994;196:123-137.

136. Fotiadis D, Kanai Y, Palacín M. The SLC3 and SLC7 families of amino acid transporters. Mol Aspects Med. 2013;34(2-3):139-158.

137. Stuart RO, Pavlova A, Beier D, Li Z, Krijanovski Y, Nigam SK. EEG1, a putative transporter expressed 
during epithelial organogenesis: comparison with embryonic transporter expression during nephrogenesis. Am J Physiol Renal Physiol 2001;281(6):F1148-F1156.

138. Bodoy S, Fotiadis D, Stoeger C, Kanai Y, Palacín M The small SLC43 family: facilitator system I amino acid transporters and the orphan EEG1. Mol Aspects Med. 2013;34(2-3):638-645.

139. Friesema EC, Docter $R$, Moerings EP, et al. Thyroid hormone transport by the heterodimeric human system L amino acid transporter. Endocrinology. 2001;142(10):4339-4348.

140. Meier C, Ristic Z, Klauser S, Verrey F. Activation of system $\mathrm{L}$ heterodimeric amino acid exchangers by intracellular substrates. Embo J. 2002;21 (4):580-589.

141. Kanai $Y$, Segawa $H$, Miyamoto Ki, Uchino $H$, Takeda E, Endou H. Expression cloning and characterization of a transporter for large neutral amino acids activated by the heavy chain of 4F2 antigen (CD98). J Biol Chem. 1998;273(37):23629-23632

142. Yan Z, Hinkle PM. Saturable, stereospecific transport of 3,5,3'-triiodo-L-thyronine and L-thyroxine into $\mathrm{GH} 4 \mathrm{C} 1$ pituitary cells. J Biol Chem. 1993;268(27):20179-20184.

143. Zhou Y, Samson M, Francon J, Blondeau JP. Thyroid hormone concentrative uptake in rat erythrocytes. Involvement of the tryptophan transport system $\mathrm{T}$ in countertransport of tri-iodothyronine and aromatic amino acids. Biochem J. 1992;281(Pt 1):81-86.

144. Everts ME, Verhoeven FA, Bezstarosti K et al. Uptake of thyroid hormones in neo natal rat cardiac myocytes. Endocrinology. 1996;137(10):4235-4242.

145. Blondeau JP, Beslin A, Chantoux F, Francon J. Triiodothyronine is a high-affinity inhibitor of amino acid transport system L1 in cultured astrocytes. J Neurochem. 1993;60(4):1407-1413.

146. Lakshmanan M, Goncalves E, Lessly G, Foti D, Robbins J. The transport of thyroxine into mouse neuroblastoma cells, NB41A3: the effect of L-system amino acids. Endocrinology. 1990;126(6):3245-3250.

147. Centanni M, Canettieri G, Viceconti N, Sibilla R, Bei A, Andreoli M. Effect of tryptophan on the early tri-iodothyronine uptake in mouse thymocytes. Eur J Endocrinol. 2000;143(1):119-123.

148. Palacín M, Estévez R, Bertran J, Zorzano A Molecular biology of mammalian plasma mem brane amino acid transporters. Physiol Rev. 1998;78(4):969-1054

149. Ritchie JW, Peter GJ, Shi YB, Taylor PM. Thyroid hormone transport by $4 \mathrm{~F} 2 \mathrm{hc}-\mathrm{IU} 12$ heterodimers expressed in Xenopus oocytes. I Endocrinol. 1999;163(2):R5-R9.

150. Morimoto E, Kanai Y, Kim DK, et al. Establishment and characterization of mammalian cell lines stably expressing human L-type amino acid transporters. J Pharmacol Sci. 2008;108(4):505-516.

151. Khunweeraphong N, Nagamori S, Wiriyasermkul P, et al. Establishment of stable cell lines with high expression of heterodimers of human $4 \mathrm{~F} 2 \mathrm{hc}$ and human amino acid transporter LAT1 or LAT2 and delineation of their differential interaction with $\alpha$-alkyl moieties. J Pharmacol Sci. 2012;119(4):368-380.

152. Kinne A, Wittner M, Wirth EK, et al. Involvement of the L-type amino acid transporter Lat2 in the transport of 3,3'-diiodothyronine across the plasma membrane. Eur Thyroid J. 2015;4(Suppl 1):42-50.

153. Hinz KM, Neef D, Rutz C, et al. Molecular features of the L-type amino acid transporter 2 determine different import and export profiles for thyroid hormones and amino acids. Mol Cell Endocrinol. 2017;443:163-174.

154. Shikano N, Kanai Y, Kawai K, Ishikawa N, Endou H. Characterization of 3-[125I]iodo-alpha-methyl-Ltyrosine transport via human L-type amino acid transporter 1. Nucl Med Biol. 2003;30(1):31-37.

155. Christensen HN. Role of amino acid transport and countertransport in nutrition and metabolism. Physiol Rev. 1990;70(1):43-77.

156. Oda K, Hosoda N, Endo H, et al. L-type amino acid transporter 1 inhibitors inhibit tumor cell growth. Cancer Sci. 2010;101(1):173-179.

157. Novak DA, Kilberg MS, Beveridge MJ. Ontogeny and plasma-membrane domain localization of amino acid transport system $L$ in rat liver. Biochem J. 1994;301 (Pt 3):671-674.

158. Nakamura $E$, Sato $M$, Yang $H$, et al. 4F2 (CD98) heavy chain is associated covalently with an amino acid transporter and controls intracellular trafficking and membrane topology of 4F2 heterodimer. J Biol Chem. 1999;274(5):3009-3016

159. Pinho MJ, Serrão MP, Gomes P, Hopfer U, Jose PA, Soares-da-Silva P. Over-expression of renal LAT1 and LAT2 and enhanced L-DOPA uptake in SHR immortalized renal proximal tubular cells. Kidney Int. 2004;66(1):216-226.

160. Rossier G, Meier C, Bauch C, et al. LAT2, a new basolateral 4F2hc/CD98-associated amino acid transporter of kidney and intestine. J Biol Chem. 1999;274(49):34948-34954.

161. Tomi M, Mori M, Tachikawa M, Katayama K, Terasaki T, Hosoya K. L-type amino acid transporter 1-mediated L-leucine transport at the inner blood-retinal barrier. Invest Ophthalmol Vis Sci. 2005;46(7):2522-2530.

162. Kageyama T, Nakamura $M$, Matsuo A, et al. The 4F2hc/LAT1 complex transports L-DOPA across the blood-brain barrier. Brain Res. 2000;879(1-2):115-121.

163. Boado RJ, Li JY, Nagaya M, Zhang C, Pardridge WM. Selective expression of the large neutral amino acid transporter at the blood-brain barrier. Proc Natl Acad Sci U S A. 1999;96(21):12079-12084.

164. Van Herck SL, Delbaere J, Bourgeois NM, McAllan BM, Richardson SJ, Darras VM. Expression of thyroid hormone transporters and deiodinases at the brain barriers in the embryonic chicken: Insights into the regulation of thyroid hormone availability during neurodevelopment. Gen Comp Endocrinol. 2015;214:30-39.

165. Umeki N, Fukasawa Y, Ohtsuki S, et al. mRNA expression and amino acid transport characteristics of cultured human brain microvascular endothelial cells (hBME). Drug Metab Pharmacokinet. 2002; 17(4):367-373.

166. Braun D, Kinne A, Bräuer AU, et al. Developmental and cell type-specific expression of thyroid hormone transporters in the mouse brain and in primary brain cells. Glia. 2011;59(3):463-471.

167. Okamoto $Y$, Sakata M, Ogura K, et al. Expression and regulation of $4 \mathrm{~F} 2 \mathrm{hc}$ and hLAT1 in human trophoblasts. Am J Physiol Cell Physiol. 2002;282(1):C196-C204.

168. Kobayashi $Y$. Localization of the system $L$ amino acid transporters LAT1 and LAT2 in rat gastrointestinal tract. J Kyorin Med Soc. 2007;38:75-84.

169. Wirth EK, Roth S, Blechschmidt C, et al. Neuronal 3,3,5-triiodothyronine (T3) uptake and behavioral phenotype of mice deficient in Mct8, the neuronal T3 transporter mutated in Allan-Herndon-Dudley syndrome. J Neurosci. 2009;29(30):9439-9449.
170. Müller J, Heuer $H$. Expression pattern of thyroid hormone transporters in the postnatal mouse brain. Front Endocrinol (Lausanne). 2014;5:92.

171. Shennan DB, Thomson J. Inhibition of system $L$ (LAT1/CD98hc) reduces the growth of cultured human breast cancer cells. Oncol Rep. 2008;20(4):885-889.

172. Onishi $Y$, Hiraiwa $M$, Kamada $H$, et al. Hypoxia affects Slc7a5 expression through HIF-2 $\alpha$ in differentiated neuronal cells. FEBS Open Bio. 2019;9(2):241-247.

173. Hayashi K, Jutabha P, Endou H, Anzai N. c-Myc is crucial for the expression of LAT1 in MIA Paca-2 human pancreatic cancer cells. Oncol Rep. 2012;28(3):862-866.

174. Halestrap AP, Meredith D. The SLC16 gene familyfrom monocarboxylate transporters (MCTs) to aromatic amino acid transporters and beyond. Pflugers Arch. 2004;447(5):619-628.

175. Rusu V, Hoch E, Mercader JM, et al; MEDIA Consortium; SIGMA T2D Consortium. Type 2 diabetes variants disrupt function of SLC16A11 through two distinct mechanisms. Cell. 2017;170(1):199-212.e20.

176. Hugo SE, Cruz-Garcia L, Karanth S, Anderson RM, Stainier DY, Schlegel A. A monocarboxylate transporter required for hepatocyte secretion of ketone bodies during fasting. Genes Dev. 2012;26(3):282-293.

177. Abplanalp J, Laczko E, Philp NJ, et al. The cataract and glucosuria associated monocarboxylate transporter MCT12 is a new creatine transporter. Hum Mol Genet. 2013;22(16):3218-3226.

178. Murakami $\mathrm{Y}$, Kohyama N, Kobayashi $\mathrm{Y}$, et al. Functional characterization of human monocarboxylate transporter 6 (SLC16A5). Drug Metab Dispos. 2005;33(12):1845-1851.

179. van Hasselt PM, Ferdinandusse S, Monroe GR, et al. Monocarboxylate transporter 1 deficiency and ketone utilization. N Engl / Med. 2014;371(20):1900-1907.

180. Lara-Riegos JC, Ortiz-López MG, Peña-Espinoza BI, et al. Diabetes susceptibility in Mayas: evidence for the involvement of polymorphisms in HHEX, HNF4 $\alpha, K C N J 11$, PPAR $\gamma$, CDKN2A/2B, SLC30A8, CDC123/CAMK1D, TCF7L2, ABCA1 and SLC16A11 genes. Gene. 2015;565(1):68-75.

181. Kloeckener-Gruissem B, Vandekerckhove K, Nürnberg G, et al. Mutation of solute carrier SLC16A12 associates with a syndrome combining juvenile cataract with microcornea and renal glucosuria. Am J Hum Genet. 2008;82(3):772-779.

182. Lafrenière RG, Carrel L, Willard HF. A novel transmembrane transporter encoded by the XPCT gene in Xa13.2. Hum Mol Genet. 1994;3(7):1133-1139.

183. Bourgeois NM, Van Herck SL, Vancamp P, et al. Characterization of chicken thyroid hormone transporters. Endocrinology. 2016;157(6):2560-2574.

184. Mughal BB, Leemans M, Lima de Souza EC, et al. Functional characterization of xenopus thyroid hormone transporters mct8 and oatp1c1. Endocrinology. 2017;158(8):2694-2705

185. Arjona FJ, de Vrieze E, Visser TJ, Flik G, Klaren PH. Identification and functional characterization of zebrafish solute carrier Slc16a2 (Mct8) as a thyroid hormone membrane transporter. Endocrinology. 2011;152(12):5065-5073.

186. Groeneweg S, Kersseboom S, van den Berge A, et al. In vitro characterization of human, mouse, and zebrafish MCT8 orthologues. Thyroid. 2019;29(10):1499-1510. 
187. Zhou Y, Samson M, Osty J, Francon J, Blondeau JP. Evidence for a close link between the thyroid hormone transport system and the aromatic amino acid transport system $T$ in erythrocytes. I Biol Chem. 1990;265(28):17000-17004.

188. Kim DK, Kanai Y, Chairoungdua A, Matsuo H, Cha SH, Endou H. Expression cloning of a $\mathrm{Na}$-independent aromatic amino acid transporter with structural similarity to $\mathrm{H}+$ / monocarboxylate transporters. I Biol Chem 2001;276(20):17221-17228.

189. van Mullem AA, van Gucht ALM, Visser WE, Meima ME, Peeters RP, Visser TJ. Effects of thyroid hormone transporters MCT8 and MCT10 on nuclear activity of T3. Mol Cell Endocrinol. 2016;437:252-260.

190. Ianculescu AG, Friesema EC, Visser T), Giacomini KM, Scanlan TS. Transport of thyroid hormones is selectively inhibited by 3-iodothyronamine. Mol Biosyst. 2010;6(8):1403-1410.

191. Visser WE, van Mullem AA, Jansen J, Visser TJ. The thyroid hormone transporters MCT8 and MCT10 transport the affinity-label $\mathrm{N}$-bromoacetyl-[(125) 1]T3 but are not modified by it. Mol Cell Endocrinol. 2011;337(1-2):96-100.

192. Kersseboom S, Horn S, Visser WE, et al. In vitro and mouse studies supporting therapeutic utility of triiodothyroacetic acid in MCT8 deficiency. Mol Endocrinol. 2014:28(12):1961-1970.

193. Horn S, Kersseboom S, Mayerl S, et al. Tetrac can replace thyroid hormone during brain development in mouse mutants deficient in the thy roid hormone transporter mct8. Endocrinology. 2013; 154(2):968-979.

194. Roth S, Kinne A, Schweizer U. The tricyclic antidepressant desipramine inhibits $\mathrm{T} 3 \mathrm{im}$ port into primary neurons. Neurosci Lett. 2010;478(1):5-8.

195. Braun D, Kim TD, le Coutre P, Köhrle J, Hershman JM, Schweizer U. Tyrosine kinase inhibitors noncompetitively inhibit MCT8mediated iodothyronine transport. I Clin Endocrinol Metab. 2012;97(1):E100-E105.

196. Illouz F, Braun D, Briet C, Schweizer U, Rodien P. Endocrine side-effects of anti-cancer drugs: thyroid effects of tyrosine kinase inhibitors. Eur J Endocrinol. 2014;171(3):R91-R99.

197. Braun D, Schweizer U. Authentic bosutinib inhibits triiodothyronine transport by monocarboxylate transporter 8. Thyroid. 2014:24(5):926-927.

198. Beukhof CM, van Doorn L, Visser TJ, et al. Sorafenib-induced changes in thyroid hormone levels in patients treated for hepatocellular carcinoma. I Clin Endocrinol Metab. 2017:102(8):2922-2929.

199. Zwanziger D, Schmidt M, Fischer J, et al. The long $\mathrm{N}$-terminus of the human monocarboxylate transporter 8 is a target of ubiquitin-dependent proteasomal degradation which regulates protein expression and oligomerization capacity. Mol Cell Endocrinol. 2016;434:278-287.

200. Nishimura M, Naito S. Tissue-specific mRNA expression profiles of human solute carrier transporter superfamilies. Drug Metab Pharmacokinet. 2008:23(1):22-44.

201. Price NT, Jackson VN, Halestrap AP. Cloning and sequencing of four new mamma lian monocarboxylate transporter (MCT) homologues confirms the existence of a transporter family with an ancient past. Biochem 1 . 1998;329 (Pt 2):321-328.
202. Loubière LS, Vasilopoulou E, Bulmer JN, et al. Expression of thyroid hormone transporters in the human placenta and changes associated with intrauterine growth restriction. Placenta. 2010:31(4):295-304

203. Alkemade A, Friesema EC, Unmehopa UA, et al. Neuroanatomical pathways for thyroid hormone feedback in the human hypothalamus. I Clin Endocrinol Metab. 2005; 90 (7):4322-4334

204. Kalló I, Mohácsik P, Vida B, et al. A novel pathway regulates thyroid hormone availability in rat and human hypothalamic neurosecretory neurons. Plos One. 2012;7(6):e37860

205. Chan SY, Hancox LA, Martín-Santos A, et al. MCT8 expression in human fetal cerebral cortex is reduced in severe intrauterine growth restriction. Endocrinol. 2014;220(2):85-95.

206. Di Cosmo C, Liao XH, Dumitrescu AM, Philp N, Weiss RE, RefetoffS. Mice deficient in MCT8 reveal a mechanism regulating thyroid hormone secretion. I Clin Invest. 2010:120(9):3377-3388.

207. Trajkovic-Arsic M, Müller J, Darras VM et al. Impact of monocarboxylate transporter-8 deficiency on the hypothalamus pituitary-thyroid axis in mice. Endocrinology. 2010;151(10):5053-5062.

208. Becker HM, Mohebbi N, Perna A, Ganapathy V Capasso G, Wagner CA. Localization of members of MCT monocarboxylate transporter family Slc16 in the kidney and regulation during metabolic acidosis. Am J Physiol Renal Physio. 2010;299(1):F141-F154.

209. Trajkovic-Arsic M, Visser TJ, Darras VM, et al. Consequences of monocarboxylate transporter 8 deficiency for renal transport and metabolism of thyroid hormones in mice. Endocrinology. 2010;151(2):802-809.

210. Henning Y, Szafranski K. Age-dependent changes of monocarboxylate transporter 8 availability in the postnatal murine retina Front Cell Neurosci. 2016:10:205.

211. Vasilopoulou E, Loubière LS, Heuer H, et al. Monocarboxylate transporter 8 modulates the viability and invasive capacity of human placental cells and fetoplacental growth in mice. Plos One. 2013;8(6):e65402.

212. Heuer $H$, Maier MK, Iden $S$, et al. The monocarboxylate transporter 8 linked to human psychomotor retardation is highly expressed in thyroid hormone-sensitive neuron populations Endocrinology. 2005;146(4):1701-1706

213. Ceballos A, Belinchon MM, Sanchez-Mendoza E, et al. Importance of monocarboxylate transporter 8 for the blood-brain barrier-dependent availability of 3,5,3'-triiodo-L-thyronine. Endocrinology. 2009:150(5):2491-2496.

214. Morte B, Gil-Ibáñez P, Bernal J. Regulation of gene expression by thyroid hormone in primary astrocytes: factors influencing the genomic response. Endocrinology. 2018;159(5):2083-2092.

215. Campinho MA, Saraiva J, Florindo C, Power DM Maternal thyroid hormones are essential for neural development in zebrafish. Mol Endocrinol. 2014:28(7):1136-1149.

216. Zada D, Tovin A, Lerer-Goldshtein T, Vatine GD, Appelbaum L. Altered behavioral performance and live imaging of circuit-specific neural deficiencies in a zebrafish model for psychomotor retardation. PloS Genet. 2014;10(9):e1004615.

217. Van Herck SL, Geysens S, Delbaere J, Tylzanowski P, Darras VM. Expression profile and thyroid hormone responsiveness of transporters and deiodinases in early embryonic chicken brain development. Mol Cell Endocrinol. 2012;349(2):289-297.

218. Zada D, Blitz E, Appelbaum L. Zebrafish-an emerging model to explore thyroid hormone transporters and psychomotor retardation. Mol Cell Endocrinol. 2017;459:53-58.

219. Lee JY, Kim MJ, Deliyanti D, et al. Overcoming monocarboxylate transporter 8 (MCT8)deficiency to promote human oligodendrocyte differentiation and myelination. Ebiomedicine. 2017;25:122-135

220. Chan SY, Martín-Santos A, Loubière LS, et al. The expression of thyroid hormone transporters in the human fetal cerebral cortex during early development and in N-Tera-2 neurodifferentiation. I Physiol. 2011;589(Pt 11):2827-2845.

221. Abe S, Namba N, Abe M, et al. Monocarboxylate transporter 10 functions as a thyroid hormone transporter in chondrocytes. Endocrinology. 2012;153(8):4049-4058.

222. Ramadan T, Camargo SM, Summa $V$, et al. Basolateral aromatic amino acid transporter TAT1 (Slc16a10) functions as an efflux pathway. J Cell Physiol. 2006;206(3):771-779.

223. Liddelow SA, Temple S, Møllgård K, et al. Molecular characterisation of transport mechanisms at the developing mouse blood-CSF interface: a transcriptome approach. PloS One. 2012;7(3):e33554.

224. Kogai T, Liu YY, Richter LL, Mody K, Kagechika H, Brent GA. Retinoic acid induces expression of the thyroid hormone transporter, monocarboxylate transporter 8 (Mct8). J Biol Chem. 2010;285(35):27279-27288.

225. Fontes KN, Cabanelas A, Bloise FF, et al. Differential regulation of thyroid hormone metabolism target genes during non-thyroidal [corrected] illness syndrome triggered by fasting or sepsis in adult mice. Front Physiol. 2017;8:828.

226. Alvarez-Salas E, Mengod G, García-Luna C, Soberanes-Chávez P, Matamoros-Trejo G, de Gortari P. Mct8 and trh co-expression throughout the hypothalamic paraventricular nucleus is modified by dehydration-induced anorexia in rats. Neuropeptides. 2016;56:33-40

227. Schutkowski A, Wege N, Stangl Gl, König B. Tissue-specific expression of monocarboxylate transporters during fasting in mice. Plos One. 2014;9(11):e112118

228. Herwig A, Campbell G, Mayer CD, et al. A thyroid hormone challenge in hypothyroid rats identifies $T 3$ regulated genes in the hypothalamus and in models with altered energy balance and glucose homeostasis. Thyroid. 2014;24(11): 1575-1593.

229. Romano RM, Gomes SN, Cardoso NC, Schiessl L, Romano MA, Oliveira CA. New insights for male infertility revealed by alterations in spermatic function and differential testicular expression of thyroid-related genes. Endocrine. 2017;55(2):607-617.

230. Badziong J, Ting S, Synoracki S, et al. Differential regulation of monocarboxylate transporter 8 expression in thyroid cancer and hyperthyroidism. Eur J Endocrinol. 2017; 177(3):243-250

231. Selmi-Ruby S, Bouazza L, Obregon MI, et al. The targeted inactivation of TR $\beta$ gene in thyroid follicular cells suggests a new mechanism of regulation of thyroid hormone production. Endocrinology. 2014; 155(2):635-646.

232. de Souza EC, Dias GR, Cardoso RC, et al. MCT8 is downregulated by short time iodine overload 
in the thyroid gland of rats. Horm Metab Res. 2015;47(12):910-915

233. Hu Z, Zhuo $X$, Shi $Y$, et al. lodine deficiency up-regulates monocarboxylate transporter 8 expression of mouse thyroid gland. Chin Med J (Engl). 2014; 127(23):4071-4076.

234. Lebsir D, Manens L, Grison S, et al. Effects of repeated potassium iodide administration on genes involved in synthesis and secretion of thyroid hormone in adult male rat. Mol Cell Endocrinol. 2018;474:119-126.

235. Serrano-Nascimento C, Salgueiro RB, Pantaleão T, Corrêa da Costa VM, Nunes MT. Maternal exposure to iodine excess throughout pregnancy and lactation induces hypothyroidism in adult male rat offspring. Sci Rep. 2017;7(1):15591.

236. Mebis L, Paletta D, Debaveye $Y$, et al. Expression of thyroid hormone transporters during critical illness. Eur J Endocrinol. 2009;161(2):243-250

237. Cubuk C, Markowsky H, Herwig A. Hypothalamic control systems show differential gene expression during spontaneous daily torpor and fasting-induced torpor in the Djungarian hamster (Phodopus sungorus). PloS One 2017;12(10):e0186299.

238. Li J, Donangelo I, Abe K, et al. Thyroid hormone treatment activates protective pathways in both in vivo and in vitro models of neuronal injury. $\mathrm{Mol}$ Cell Endocrinol. 2017:452:120-130.

239. Engels K, Rakov H, Zwanziger D, et al. Differences in mouse hepatic thyroid hormone transporter expression with age and hyperthyroidism. Eur Thyroid J. 2015;4(Suppl 1):81-86

240. Forrest LR, Krämer R, Ziegler C. The structural basis of secondary active transport mechanisms. Biochim Biophys Acta. 2011; 1807(2):167-188

241. Gao X, Zhou L, Jiao X, et al. Mechanism of substrate recognition and transport by an amino acid antiporter. Nature. 2010;463(7282):828-832.

242. Schweizer U, Johannes J, Bayer D, Braun D. Structure and function of thyroid hormone plasma membrane transporters. Eur Thyroid J 2014;3(3):143-153.

243. Poole RC, Sansom CE, Halestrap AP. Studies of the membrane topology of the rat erythrocyte $\mathrm{H}+$ /lactate cotransporter (MCT1). Biochem J. 1996;320 (Pt 3):817-824

244. Huang Y, Lemieux MJ, Song J, Auer M, Wang DN. Structure and mechanism of the glycerol-3phosphate transporter from Escherichia coli. Science. 2003;301(5633):616-620.

245. Groeneweg S, Friesema EC, Kersseboom S, et al. The role of Arg 445 and Asp498 in the human thyroid hormone transporter MCT8. Endocrinology. 2014; 155(2):618-626

246. Nascimento AS, Dias SM, Nunes FM, et al. Structural rearrangements in the thyroid hormone receptor hinge domain and their putative role in the receptor function. J Mol Biol. 2006;360(3):586-598.

247. Schweizer U, Schlicker C, Braun D, Köhrle J, Steegborn C. Crystal structure of mammalian selenocysteine-dependent iodothyronine deiodinase suggests a peroxiredoxin-like catalytic mechanism. Proc Natl Acad Sci U S A. 2014;111(29):10526-10531.

248. Kleinau G, Schweizer U, Kinne A, et al. Insights into molecular properties of the human monocarboxylate transporter 8 by combining functional with structural information. Thyroid Res. 2011;4(Suppl 1):S4.
249. Braun D, Lelios I, Krause G, Schweizer U. Histidines in potential substrate recognition sites affect thyroid hormone transport by monocarboxylate transporter 8 (МСT8). Endocrinology. 2013;154(7):2553-2561.

250. Groeneweg S, Lima de Souza EC, Visser WE, Peeters RP, Visser TJ. Importance of His192 in the human thyroid hormone transporter MCT8 for substrate recognition. Endocrinology. 2013;154(7):2525-2532.

251. Lima de Souza EC, Groeneweg S, Visser WE, Peeters RP, Visser TJ. Importance of cysteine residues in the thyroid hormone transporter MCT8. Endocrinology. 2013;154(5):1948-1955.

252. Protze J, Braun D, Hinz KM, Bayer-Kusch D, Schweizer $U$, Krause G. Membrane-traversing mechanism of thyroid hormone transport by monocarboxylate transporter 8. Cell Mol Life Sci. 2017;74(12):2299-2318.

253. Groeneweg S, Lima de Souza EC, Meima ME, Peeters RP, Visser WE, Visser TJ. Outwardopen model of thyroid hormone transporter monocarboxylate transporter 8 provides novel structural and functional insights. Endocrinology. 2017;158(10):3292-3306.

254. Dang S, Sun L, Huang Y, et al. Structure of a fucose transporter in an outward-open conformation. Nature. 2010;467(7316):734-738.

255. Braun D, Schweizer U. Thyroid hormone transport and transporters. Vitam Horm. 2018;106:19-44.

256. Uemura S, Mochizuki T, Kurosaka G, Hashimoto T, Masukawa $Y$, Abe F. Functional analysis of human aromatic amino acid transporter MCT10/TAT1 using the yeast Saccharomyces cerevisiae. Biochim Biophys Acta Biomembr. 2017;1859(10):2076-2085.

257. Rechsteiner $M$, Rogers SW. PEST sequences and regulation by proteolysis. Trends Biochem Sci. 1996;21(7):267-271.

258. Kirk P, Wilson MC, Heddle C, Brown MH, Barclay AN, Halestrap AP. CD147 is tightly associated with lactate transporters MCT1 and MCT4 and facilitates their cell surface expression. Embo J. 2000;19(15):3896-3904.

259. Visser WE, Philp NJ, van Dijk TB, et al. Evidence for a homodimeric structure of human monocarboxylate transporter 8. Endocrinology. 2009;150(11):5163-5170.

260. Fischer J, Kleinau G, Müller A, et al. Modulation of monocarboxylate transporter 8 oligomerization by specific pathogenic mutations. J Mol Endocrinol. 2015;54(1):39-50

261. Biebermann $\mathrm{H}$, Ambrugger $\mathrm{P}$, Tarnow P, von Moers A, Schweizer U, Grueters A. Extended clinical phenotype, endocrine investigations and functional studies of a loss-of-function mutation A150V in the thyroid hormone specific transporter MCT8. Eur J Endocrinol. 2005; 153(3):359-366.

262. Smith VE, Read ML, Turnell AS, et al. PTTGbinding factor (PBF) is a novel regulator of the thyroid hormone transporter MCT8. Endocrinology. 2012;153(7):3526-3536.

263. Fischer J, Kleinau G, Rutz C, et al. Evidence of $\mathrm{G}$-protein-coupled receptor and substrate transporter heteromerization at a single molecule level. Cell Mol Life Sci. 2018;75(12):2227-2239.

264. Schlessinger A, Yee SW, Sali A, Giacomini KM. SLC classification: an update. Clin Pharmacol Ther. 2013;94(1):19-23.

265. Rosell A, Meury M, Álvarez-Marimon E, et al. Structural bases for the interaction and stabilization of the human amino acid transporter LAT2 with its ancillary protein 4F2hc. Proc Natl Acad Sci U S A. 2014;111(8):2966-2971.

266. Fort J, de la Ballina LR, Burghardt HE, et al. The structure of human $4 \mathrm{~F} 2 \mathrm{hc}$ ectodomain provides a model for homodimerization and electrostatic interaction with plasma membrane. J Biol Chem. 2007;282(43):31444-31452.

267. Meury M, Costa M, Harder D, et al. Detergentinduced stabilization and improved 3D map of the human heteromeric amino acid transporter 4F2hc-LAT2. Plos One. 2014;9(10):e109882.

268. Yan R, Zhao X, Lei J, Zhou Q. Structure of the human LAT1-4F2hc heteromeric amino acid transporter complex. Nature. 2019;568(7750):127-130.

269. Geier EG, Schlessinger A, Fan H, et al. Structurebased ligand discovery for the large-neutral amino acid transporter 1, LAT-1. Proc Natl Acad Sci U S A. 2013;110(14):5480-5485.

270. Augustyn E, Finke K, Zur AA, et al. LAT-1 activity of meta-substituted phenylalanine and tyrosine analogs. Bioorg Med Chem Lett. 2016;26(11):2616-2621.

271. Zur AA, Chien HC, Augustyn E, et al. LAT1 activity of carboxylic acid bioisosteres: evaluation of hydroxamic acids as substrates. Bioorg Med Chem Lett. 2016;26(20):5000-5006

272. Napolitano L, Galluccio $M$, Scalise $M$, et al. Novel insights into the transport mechanism of the human amino acid transporter LAT1 (SLC7A5). Probing critical residues for substrate translocation. Biochim Biophys Acta Gen Subj. 2017; 1861(4):727-736

273. Napolitano L, Scalise M, Koyioni M, et al. Potent inhibitors of human LAT1 (SLC7A5) transporter based on dithiazole and dithiazine compounds for development of anticancer drugs. Biochem Pharmacol. 2017;143:39-52.

274. Kowalczyk L, Ratera M, Paladino A, et al. Molecular basis of substrate-induced permeation by an amino acid antiporter. Proc Natl Acad Sci U S A. 2011;108(10):3935-3940

275. Shaffer PL, Goehring A, Shankaranarayanan A, Gouaux E. Structure and mechanism of a $\mathrm{Na+}$ independent amino acid transporter. Science. 2009;325(5943):1010-1014.

276. Hinz KM, Meyer K, Kinne A, Schülein R, Köhrle J, Krause G. Structural insights into thyroid hormone transport mechanisms of the L-type amino acid transporter 2. Mol Endocrinol. 2015;29(6):933-942.

277. Krause G, Hinz KM. Thyroid hormone transport across L-type amino acid transporters: what can molecular modelling tell us? Mol Cell Endocrinol. 2017;458:68-75

278. Wang P, Hata S, Xiao Y, et al. Topological assessment of oatp1a1: a 12-transmembrane domain integral membrane protein with three $\mathrm{N}$-linked carbohydrate chains. Am J Physiol Gastrointest Liver Physiol. 2008;294(4):G1052-G1059.

279. Meier-Abt F, Mokrab Y, Mizuguchi K. Organic anion transporting polypeptides of the OATP/ SLCO superfamily: identification of new members in nonmammalian species, comparative modeling and a potential transport mode. J Membr Biol. 2005;208(3):213-227.

280. Hänggi E, Grundschober AF, Leuthold S, Meier PJ, St-Pierre MV. Functional analysis of the extracellular cysteine residues in the human organic anion transporting polypeptide, OATP2B1. Mol Pharmacol. 2006;70(3):806-817.

281. Lee TK, Koh AS, Cui Z, Pierce RH, Ballatori N. $\mathrm{N}$-glycosylation controls functional activity of 
Oatp1, an organic anion transporter. Am J Physiol Gastrointest Liver Physiol. 2003;285(2):G371-G381.

282. Gui C, Hagenbuch B. Amino acid residues in transmembrane domain 10 of organic anion transporting polypeptide $1 \mathrm{~B} 3$ are critical for cholecystokinin octapeptide transport. Biochemistry. 2008;47(35):9090-9097.

283. Miyagawa M, Maeda K, Aoyama A, Sugiyama Y. The eighth and ninth transmembrane domains in organic anion transporting polypeptide 1B1 affect the transport kinetics of estrone-3-sulfate and estradiol-17beta-D-glucuronide. J Pharmacol Exp Ther. 2009;329(2):551-557.

284. DeGorter MK, Ho RH, Leake BF, Tirona RG, Kim RB. Interaction of three regiospecific amino acid residues is required for OATP1B1 gain of OATP1B3 substrate specificity. Mol Pharm. 2012;9(4):986-995.

285. Gui C, Hagenbuch B. Role of transmembrane domain 10 for the function of organic anion transporting polypeptide 1B1. Protein Sci. 2009; 18(11):2298-2306.

286. Li $\mathrm{N}$, Hong $\mathrm{W}$, Huang $\mathrm{H}$, Lu $\mathrm{H}$, Lin $\mathrm{G}$, Hong $\mathrm{M}$. Identification of amino acids essential for estrone3-sulfate transport within transmembrane domain 2 of organic anion transporting polypeptide 1B1. PloS One. 2012; 7(5):e36647.

287. Hong W, Wu Z, Fang Z, Huang J, Huang $H$, Hong $M$. Amino acid residues in the putative transmembrane domain 11 of human organic anion transporting polypeptide $1 \mathrm{~B} 1$ dictate transporter substrate binding, stability, and trafficking. Mol Pharm. 2015;12(12):4270-4276.

288. Glaeser H, Mandery K, Sticht H, Fromm MF König J. Relevance of conserved lysine and arginine residues in transmembrane helices for the transport activity of organic anion transporting polypeptide 1B3. Br J Pharmacol. 2010;159(3):698-708

289. Mandery K, Sticht H, Bujok K, et al. Functional and structural relevance of conserved positively charged lysine residues in organic anion transporting polypeptide 1B3. Mol Pharmacol. 2011;80(3):400-406.

290. Weaver YM, Hagenbuch B. Several conserved positively charged amino acids in OATP1B1 are involved in binding or translocation of different substrates. I Membr Biol. 2010;236(3):279-290.

291. Huang J, Li N, Hong W, et al. Conserved tryptophan residues within putative transmembrane domain 6 affect transport function of organic anion transporting polypeptide 1B1. Mol Pharmacol. 2013;84(4):521-527.

292. Chan T, Zheng J, Zhu L, Grewal T, Murray M, Zhou F. Putative transmembrane domain 6 of the human organic anion transporting polypeptide 1A2 (OATP1A2) influences transporter substrate binding, protein trafficking, and quality control. Mol Pharm. 2015;12(1):111-119.

293. Bian J, Jin $M$, Yue $M$, Wang $M$, Zhang H, Gui C. Tryptophan residue located at the middle of putative transmembrane domain 11 is critical for the function of organic anion transporting polypeptide 2B1. Mol Pharm. 2016;13(10):3553-3563.

294. Fang Z, Huang J, Chen J, Xu S, Xiang Z, Hong M. Transmembrane domain 1 of human organic anion transporting polypeptide $2 \mathrm{~B} 1$ is essential for transporter function and stability. Mol Pharmacol. 2018;94(2):842-849.

295. Westholm DE, Marold JD, Viken KJ, et al. Evidence of evolutionary conservation of function between the thyroxine transporter Oatp1c1 and major facilitator superfamily members. Endocrinology 2010;151(12):5941-5951.

296. Heng J, Zhao Y, Liu M, et al. Substrate-bound structure of the E. coli multidrug resistance transporter MdfA. Cell Res. 2015;25(9):1060-1073.

297. Campos IT, Souza TA, Torquato RI, et al. The Kazal-type inhibitors infestins 1 and 4 differ in specificity but are similar in three-dimensional structure. Acta Crystallogr D Biol Crystallogr. 2012;68(Pt 6):695-702.

298. Hu NJ, Iwata S, Cameron AD, Drew D. Crystal structure of a bacterial homologue of the bile acid sodium symporter ASBT. Nature. 2011;478(7369):408-411.

299. Porcu E, Medici M, Pistis G, et al. A meta-analysis of thyroid-related traits reveals novel loci and genderspecific differences in the regulation of thyroid function. Plos Genet. 2013;9(2):e1003266.

300. van der Deure WM, Peeters RP, Visser TJ. Genetic variation in thyroid hormone transporters. Best Pract Res Clin Endocrinol Metab. 2007;21(2):339-350.

301. Lago-Lestón R, Iglesias MJ, San-José E, et al. Prevalence and functional analysis of the S107P polymorphism (rs6647476) of the monocarboxylate transporter 8 (SLC16A2) gene in the male population of north-west Spain (Galicia). Clin Endocrinol (Oxf). 2009;70(4):636-643.

302. Roef GL, Rietzschel ER, De Meyer T, et al. Associations between single nucleotide polymorphisms in thyroid hormone transporter genes (MCT8, MCT10 and OATP1C1) and circulating thyroid hormones. Clin Chim Acta. 2013;425:227-232

303. Medici $M$, van der Deure WM, Verbiest $M$, et al. A large-scale association analysis of 68 thyroid hormone pathway genes with serum TSH and FT4 levels. Eur J Endocrinol. 2011; 164(5):781-788

304. Carlé A, Faber J, Steffensen R, Laurberg P, Nygaard B. Hypothyroid patients encoding combined MCT10 and DIO2 gene polymorphisms may prefer $\mathrm{L}-\mathrm{T} 3+\mathrm{L}-\mathrm{T} 4$ combination treatmentdata using a blind, randomized, clinical Study. Eur Thyroid J. 2017;6(3):143-151.

305. Badagnani I, Castro RA, Taylor TR, et al. Interaction of methotrexate with organic-anion transporting polypeptide $1 \mathrm{~A} 2$ and its genetic variants. I Pharmacol Exp Ther. 2006;318(2):521-529.

306. Smith NF, Figg WD, Sparreboom A. Role of the liver-specific transporters OATP1B1 and OATP1B3 in governing drug elimination. Expert Opin Drug Metab Toxicol. 2005;1(3):429-445.

307. Niemi M, Backman JT, Kajosaari LI, et al. Polymorphic organic anion transporting polypeptide $1 \mathrm{~B} 1$ is a major determinant of repaglinide pharmacokinetics. Clin Pharmacol Ther. 2005; $77(6): 468-478$

308. Lee HK, Hu M, Lui SSh, Ho CS, Wong CK, Tomlinson B. Effects of polymorphisms in ABCG2, SLCO1B1, SLC10A1 and CYP2C9/19 on plasma concentrations of rosuvastatin and lipid response in Chinese patients. Pharmacogenomics. 2013;14(11):1283-1294.

309. van der Deure WM, Appelhof BC, Peeters RP, et al. Polymorphisms in the brain-specific thyroid hormone transporter OATP1C1 are associated with fatigue and depression in hypothyroid patients. Clin Endocrinol (Oxf). 2008;69(5):804-811.

310. Brozaitiene J, Skiriute D, Burkauskas J, et al. Deiodinases, organic anion transporter polypeptide polymorphisms, and thyroid hormones in patients with myocardial infarction. Genet Test $\mathrm{Mol}$ Biomarkers. 2018;22(4):270-278.

311. Ho RH, Leake BF, Roberts RL, Lee W, Kim RB. Ethnicity-dependent polymorphism in $\mathrm{Na}$-taurocholate cotransporting polypeptide (SLC10A1) reveals a domain critical for bile acid substrate recognition. I Biol Chem. 2004;279(8):7213-7222.

312. Boado RJ, Li JY, Wise P, Pardridge WM. Human LAT1 single nucleotide polymorphism N230K does not alter phenylalanine transport. Mol Genet Metab. 2004;83(4):306-311.

313. Allan W, Herndon CN, Dudley FC. Some examples of the inheritance of mental deficiency: apparently sex-linked idiocy and microcephaly. Am J Ment Defic. 1944;48:325-334.

314. Schwartz CE, Ulmer J, Brown A, Pancoast I, Goodman HO, Stevenson RE. Allan-Herndon syndrome. II. Linkage to DNA markers in Xq21. Am J Hum Genet. 1990;47(3):454-458.

315. Stevenson RE, Goodman HO, Schwartz $C E$, Simensen RJ, McLean WT Jr, Herndon CN. AllanHerndon syndrome. I. Clinical studies. Am J Hum Genet. 1990;47(3):446-453.

316. Passos-Bueno MR, Byth BC, Rosenberg S, et al. Severe nonspecific $X$-linked mental retardation caused by a proximally Xp located gene: intragenic heterogeneity or a new form of X-linked mental retardation? Am J Med Genet. 1993;46(2):172-175.

317. Davis JG, Silverber G, Williams MK, Spiro, A, Shapiro LR. A new $X$-linked recessive mental retardation syndrome with progressive spastic quadriparesis. Am J Hum Genet. 1981;33:75A.

318. Zorick TS, Kleimann S, Sertié A, Zatz M, Rosenberg S, Passos-Bueno MR. Fine mapping and clinical reevaluation of a Brazilian pedigree with a severe form of $\mathrm{X}$-linked mental retardation associated with other neurological dysfunction. Am J Med Genet A. 2004;127A(3):321-323.

319. Bialer MG, Lawrence L, Stevenson RE, et al. AllanHerndon-Dudley syndrome: clinical and linkage studies on a second family. Am । Med Genet. 1992;43(1-2):491-497.

320. Maranduba CM, Friesema EC, Kok F, et al. Decreased cellular uptake and metabolism in Allan-Herndon-Dudley syndrome (AHDS) due to a novel mutation in the MCT8 thyroid hormone transporter. J Med Genet. 2006;43(5):457-460.

321. Schwartz CE, May MM, Carpenter NJ, et al. Allan-Herndon-Dudley syndrome and the monocarboxylate transporter 8 (MCT8) gene. Am J Hum Genet. 2005;77(1):41-53.

322. Choi JH, Cho JH, Kim JH, Yoo EG, Kim GH, Yoo HW. Variable clinical characteristics and molecular spectrum of patients with syndromes of reduced sensitivity to thyroid hormone: genetic defects in the THRB and SLC16A2 genes. Horm Res Paediatr. 2018;90(5):283-290.

323. Kim JH, Kim YM, Yum MS, et al. Clinical and endocrine features of two Allan-Herndon-Dudley syndrome patients with monocarboxylate transporter 8 mutations. Horm Res Paediatr. 2015;83(4):288-292

324. Capri Y, Friesema EC, Kersseboom S, et al. Relevance of different cellular models in determining the effects of mutations on SLC16A2/ MCT8 thyroid hormone transporter function and genotype-phenotype correlation. Hum Mutat. 2013;34(7):1018-1025.

325. Shimojima K, Maruyama K, Kikuchi M, Imai A, Inoue K, Yamamoto T. Novel SLC16A2 mutations 
in patients with Allan-Herndon-Dudley syndrome Intractable Rare Dis Res. 2016;5(3):214-217.

326. Islam MS, Namba N, Ohata Y, et al. Functional analysis of monocarboxylate transporter 8 mutations in Japanese Allan-Herndon-Dudley syndrome patients. Endocr J. 2019;66(1):19-29.

327. Ono $E$, Ariga $M$, Oshima $S$, et al. Three novel mutations of the MCT8 (SLC16A2) gene: individual and temporal variations of endocrinological and radiological features. Clin Pediatr Endocrinol. 2016;25(2):23-35.

328. Grozeva D, Carss K, Spasic-Boskovic O, et al; Italian X-linked Mental Retardation Project; UK10K Consortium; GOLD Consortium. Targeted next-generation sequencing analysis of 1,000 individuals with intellectual disability. Hum Mutat. 2015;36(12):1197-1204

329. Thevenon J, Duffourd Y, Masurel-Paulet A, et al. Diagnostic odyssey in severe neurodevelopmental disorders: Towards clinical whole-exome sequencing as a first-line diagnostic test. Clin Genet. 2016;89(6):700-707

330. Vaurs-Barrière $C$, Deville $M$, Sarret $C$, et al. Pelizaeus-Merzbacher-Like disease presentation of MCT8 mutated male subjects. Ann Neurol. 2009;65(1):114-118.

331. Raymond F, Whibley A, Price S, et al. Raised T3 levels and mutations in MCT8 (SLC16A2) cause $X$-linked cerebral palsy and mental retardation. Eur J Med Genet. 2008;14(Suppl 1):60.

332. Boccone L, Dessì V, Meloni A, Loudianos G. AllanHerndon-Dudley syndrome (AHDS) in two consecutive generations caused by a missense MCT8 gene mutation. Phenotypic variability with the presence of normal serum T3 levels. Eur J Med Genet. 2013;56(4):207-210.

333. Yamamoto $T$, Shimojima $K$, Umemura $A$, Uematsu M, Nakayama T, Inoue K. SLC16A2 mutations in two Japanese patients with AllanHerndon-Dudley syndrome. Human Genome Variation. 2014;1:14010.

334. Friesema EC, Jansen J, Heuer $H$, Trajkovic $M$, Bauer K, Visser TJ. Mechanisms of disease: psychomotor retardation and high T3 levels caused by mutations in monocarboxylate transporter 8. Nat Clin Pract Endocrinol Metab. 2006;2(9):512-523.

335. Jansen J, Friesema EC, Kester $M H$, et al. Functional analysis of monocarboxylate transporter 8 mutations identified in patients with $X$-linked psychomotor retardation and elevated serum triiodothyronine. J Clin Endocrinol Metab. 2007;92(6):2378-2381.

336. Yamamoto T, Lu Y, Nakamura R, Shimojima $K$, Kira R. Novel A178P mutation in SLC16A2 in a patient with Allan-Herndon-Dudley syndrome. Congenit Anom (Kyoto). 2018;58(4):143-144.

337. Fuchs O, Pfarr N, Pohlenz J, Schmidt H. Elevated serum triiodothyronine and intellectual and motor disability with paroxysmal dyskinesia caused by a monocarboxylate transporter 8 gene mutation. Dev Med Child Neurol. 2009;51(3):240-244.

338. Hu H, Wrogemann K, Kalscheuer V, et al. Mutation screening in 86 known $X$-linked mental retardation genes by droplet-based multiplex PCR and massive parallel sequencing. Hugo J. 2009;3 (1-4):41-49.

339. Tzschach A, Grasshoff U, Beck-Woedl S, et al. Nextgeneration sequencing in $\mathrm{X}$-linked intellectual disability. Eur J Hum Genet. 2015;23(11):1513-1518.

340. Novara F, Groeneweg S, Freri E, et al. Clinical and molecular characteristics of SLC16A2 (MCT8) mutations in three families with the Allan-Herndon-Dudley syndrome. Hum Mutat. 2017;38(3):260-264.
341. Wémeau JL, Pigeyre $M$, Proust-Lemoine $E$, et al. Beneficial effects of propylthiouracil plus L-thyroxine treatment in a patient with a mutation in MCT8. J Clin Endocrinol Metab. 2008;93(6):2084-2088.

342. Dateki S, Haraguchi K, Sato T, et al. A novel MCT8 mutation in a Japanese patient with Allan-Herndon-Dudley syndrome. Horm Res. 2013;80(Suppl 1):360.

343. Wood T, Hobson D, Browning B, et al. The utilization of T3/T4 screening of males with MR of unknown etiology to identify patients with AllanHerndon-Dudley syndrome. Eur I Hum Genet. 2008;16(Suppl 1):26.

344. Ngu LH. Developmental delay and abnormal thyroid function test in two siblings caused by a novel mutation in SLC16A2 gene affecting a thyroid hormone specific transporter (MCT8): first report from Malaysia. Conference abstract: 35 th Malaysian Paediatrics Association Congress; 27-30 June 2013, Kuantan, Pahang, Malaysia.

345. Iglesias Álvarez A; Gómez-Gila AL; Casano P; del Pozo J, de Mingo MC, Pons N. Respuesta hormonal al tratamiento con triac en 5 niños españoles con síndrome de allan-herndon-dudley por defecto en el transportador mct8 de hormonas tiroideas. Rev Esp Endocrinol Pediatr. 2015;6(Suppl 1):91-3.

346. Armour CM, Kersseboom S, Yoon G, Visser TJ. Further insights into the Allan-Herndon-Dudley syndrome: clinical and functional characterization of a novel MCT8 mutation. PLoS One. 2015;10(10):e0139343.

347. La Piana R, Vanasse $M$, Brais B, Bernard G. Myelination delay and Allan-Herndon-Dudley syndrome caused by a novel mutation in the SLC16A2 gene. J Child Neurol. 2015;30(10):1371-1374.

348. Noguchi A, Takahashi I, Shoji Y, Oyamada M, Takahashi T. Transient acidosis in infancy with a novel variant in MCT8 (Monocarboxylate transporter 8) gene. Horm Res. 2009;72(Suppl 1):310-1.

349. Gika AD, Siddiqui A, Hulse AJ, et al. White matter abnormalities and dystonic motor disorder associated with mutations in the SLC16A2 gene. Dev Med Child Neurol. 2010;52(5):475-482

350. Langley KG, Trau S, Bean LJ, Narravula A, Schrier Vergano SA. A 7-month-old male with Allan-Herndon-Dudley syndrome and the power of T3. Am J Med Genet A. 2015;167A(5):1117-1120.

351. Fu J, Refetoff S, Dumitrescu AM, Weiss RE. OR293: whole-exome sequencing identified a novel MCT8 gene mutation in a child with mild cognitive, motor and behavior abnormalities. Endocr Rev. 2014;35:OR29-OR33.

352. Faruk Aydin O, Kara C, Jones J, et al. AllanHerndon-Dudley syndrome caused by a novel MCT8/SLC16A2 mutation in a Turkish family. Horm Res. 2013;80(Suppl 1):352-353.

353. Philips AK, Sirén A, Avela K, et al. X-exome sequencing in Finnish families with intellectual disability - four novel mutations and two novel syndromic phenotypes. Orphanet J Rare Dis. 2014;9:49

354. Ono E, Ariga M, Oshima S, et al. Endocrinological investigations in two cases of MCT8 abnormality with novel mutations in the SLC16A2 gene. 95th Annual Meeting of The Endocrine Society; June 15-18, 2013, San Francisco, CA.

355. Tonduti D, Vanderver A, Berardinelli A, et al. MCT8 deficiency: extrapyramidal symptoms and delayed myelination as prominent features. J Child Neurol. 2013;28(6):795-800

356. Redin C, Gérard B, Lauer J, et al. Efficient strategy for the molecular diagnosis of intellectual disability using targeted high-throughput sequencing. I Med Genet. 2014;51(11):724-736.

357. Visser WE, Vrijmoeth P, Visser FE, Arts WF, van Toor $H$, Visser T). Identification, functional analysis, prevalence and treatment of monocarboxylate transporter 8 (MCT8) mutations in a cohort of adult patients with mental retardation. Clin Endocrinol (Oxf). 2013;78(2):310-315.

358. López-Espíndola D, Morales-Bastos C, GrijotaMartínez C, et al. Mutations of the thyroid hormone transporter MCT8 cause prenatal brain damage and persistent hypomyelination. J Clin Endocrinol Metab. 2014:99(12):E2799-E2804.

359. Anık A, Kersseboom S, Demir K, et al. Psychomotor retardation caused by a defective thyroid hormone transporter: report of two families with different MCT8 mutations. Horm Res Paediatr. 2014;82(4):261-271.

360. Ugrasbul F, Ardinger $\mathrm{HH}$. A patient presenting with central hypothyroidism, developmental delay and poor head control. Should we be checking T3 levels? Horm Res. 2009;72(Suppl 1):458-459.

361. Brockmann K, Dumitrescu AM, Best TT, Hanefeld F, Refetoff S. X-linked paroxysmal dyskinesia and severe global retardation caused by defective MCT8 gene. J Neurol. 2005;252(6):663-666.

362. Rego T, Lado CG, Rodríguez PC, et al. Severe neurological abnormalities in a young boy with impaired thyroid hormone sensitivity due to a novel mutation in the MCT8 gene. Hormones (Athens). 2017;16(2):194-199.

363. Papadimitriou A, Dumitrescu AM, Papavasiliou A, Fretzayas A, Nicolaidou P, Refetoff S. A novel monocarboxylate transporter 8 gene mutation as a cause of severe neonatal hypotonia and developmental delay. Pediatrics. 2008;121(1):e199-e202.

364. Kobayashi S, Onuma A, Inui $T$, et al. Clinical course and images of four familial cases of Allan-Herndon-Dudley syndrome with a novel monocarboxylate transporter 8 gene mutation. Pediatr Neurol. 2014;51(3):414-416.

365. Frints SG, Lenzner S, Bauters M, et al. MCT8 mutation analysis and identification of the first female with Allan-Herndon-Dudley syndrome due to loss of MCT8 expression. Eur J Hum Genet. 2008; 16(9):1029-1037.

366. Visser WE, Jansen J, Friesema EC, et al. Novel pathogenic mechanism suggested by ex vivo analysis of MCT8 (SLC16A2) mutations. Hum Mutat. 2009;30(1):29-38.

367. Sijens PE, Rödiger LA, Meiners LC, Lunsing RJ. $1 \mathrm{H}$ magnetic resonance spectroscopy in monocarboxylate transporter 8 gene deficiency. J Clin Endocrinol Metab. 2008;93(5):1854-1859.

368. Tasdelen $\mathrm{E}, \mathrm{Bas} \mathrm{V}$, Aslan $\mathrm{H}$, et al. A novel mutation in SLC16A2 gene in a Turkish boy with AllanHerndon-Dudley Syndrome. Eur I Hum Genet. 2016;24(Suppl 1):P10.01A.

369. Wang J, Zhang Q, Bao X, Chen Y, Yu S. Clinical and genetic features of five patients with AllanHerndon-Dudley syndrome. Zhonghua Yi Xue Yi Chuan Xue Za Zhi. 2018;35(4):484-488.

370. Swiston C), Nash DL. Intermittent esotropia in 4 patients with Allan-Herndon-Dudley syndrome. J Child Neurol. 2018;33(8):525-527.

371. Goto $M$, Ito K, Okamoto $N$, Sato N, Sasaki $M$. Cerebral blood flow on (99m)Tc ethyl cysteinate dimer SPECT in 2 siblings with monocarboxylate transporter 8 deficiency. Clin Nucl Med. 2013;38(6):e276-e278.

372. Bedoyan JK, Tim-Aroon T, Deeb KK, Ganganna ST, Bass NE. The value of comprehensive thyroid function testing and family history for early 
diagnosis of MCT8 deficiency. Clin Pediatr (Phila) 2016;55(3):286-289.

373. Holden KR, Zuñiga OF, May MM, et al. X-linked MCT8 gene mutations: characterization of the pediatric neurologic phenotype. J Child Neurol. 2005;20(10):852-857

374. Filho HC, Marui S, Manna TD, et al. Novel mutation in MCT8 gene in a Brazilian boy with thyroid hormone resistance and severe neurologic abnormalities. Ara Bras Endocrinol Metabol. 2011;55(1):60-66.

375. Kakinuma $H$, Itoh $M$, Takahashi $H$. A novel mutation in the monocarboxylate transporter 8 gene in a boy with putamen lesions and low free T4 levels in cerebrospinal fluid. I Pediatr. 2005; 147(4):552-554.

376. Verge CF, Konrad D, Cohen $M$, et al Diiodothyropropionic acid (DITPA) in the treatment of MCT8 deficiency. J Clin Endocrinol Metab. 2012;97(12):4515-4523.

377. Rodrigues F, Grenha J, Ortez C, et al. Hypotonic male infant and MCT8 deficiency-a diagnosis to think about. BMC Pediatr. 2014; 14:252.

378. Levenson A, Tan WH, Huang SA. Diagnostic dilemma: a 3-year old boy with global developmental delay, truncal hypotonia, peripheral hypertonia, and central hypothyroidism. 95th annual meeting of the Endocrine Society; June 15-18, 2013, San Francisco, CA.

379. Herzovich V, Vaiani E, Marino R, et al. Unexpected peripheral markers of thyroid function in a patient with a novel mutation of the MCT8 thyroid hormone transporter gene. Horm Res. 2007:67(1):1-6.

380. Tsurusaki Y, Osaka H, Hamanoue $H$, et al. Rapid detection of a mutation causing $X$-linked leucoencephalopathy by exome sequencing. J Med Genet. 2011;48(9):606-609.

381. Azzolini S, Nosadini M, Balzarin M, et al. Delayed myelination is not a constant feature of AllanHerndon-Dudley syndrome: report of a new case and review of the literature. Brain Dev. 2014;36(8):716-720.

382. Yamamoto T, Takanashi J, Kurosawa K, Deguchi K, Osaka H, Inoue K. Comment on "delayed myelination is not a constant feature of Allan-HerndonDudley syndrome: report of a new case and review of the literature" by Azzolini $S$ et al. Brain \& Development 2014;36:716-720. Brain Dev. 2015;37(10):988-989.

383. Boccone L, Mariotti S, Dessì V, Pruna D, Meloni A, Loudianos G. Allan-HerndonDudley syndrome (AHDS) caused by a novel SLC16A2 gene mutation showing severe neurologic features and unexpectedly low TRH-stimulated serum TSH. Eur J Med Genet. 2010;53(6):392-395.

384. Crushell E, Reardon W. Elevated TSH levels in a mentally retarded boy. Eur J Pediatr. 2010;169(5):573-575.

385. Namba N, Etani Y, Kitaoka T, et al. Clinical phenotype and endocrinological investigations in a patient with a mutation in the MCT8 thyroid hormone transporter. Eur J Pediatr. 2008;167(7):785-791.

386. Garcia-de Teresa B, Gonzalez-Del Angel A, Reyna-Fabian ME, et al. Deletion of exon 1 of the SLC16A2 gene: a common occurrence in patients with Allan-Herndon-Dudley syndrome. Thyroid. 2015;25(3):361-367.

387. Zung A, Visser TJ, Uitterlinden AG, Rivadeneira F, Friesema EC. A child with a deletion in the monocarboxylate transporter 8 gene: 7-year follow-up and effects of thyroid hormone treatment. Eur J Endocrinol. 2011;165(5):823-830.

388. Yamamoto S, Okuhara K, Tonoki H, lizuka S, Nihei N, Tajima T. A novel deletion mutation of SLC16A2 encoding monocarboxylate transporter (MCT) 8 in a 26-year-old Japanese patient with Allan-Herndon-Dudley syndrome. Clin Pediatr Endocrinol. 2013;22(4):83-86.

389. Tang $\mathrm{YL}$, Peng J, Xiong J, et al. A family with Allan-Herndon-Dudley syndrome due to SLC16A2 gene mutation. Zhonghua Er Ke Za Zhi. 2018;56(11):829-834.

390. Gagliardi L, Nataren N, Feng J, et al. AllanHerndon-Dudley syndrome with unusual profound sensorineural hearing loss. Am J Med Genet A. 2015;167A(8):1872-1876.

391. López-Marín L, Martín-Belinchón M, GutiérrezSolana LG, Morte-Molina B, Duat-Rodríguez A, Bernal J. MCT8-specific thyroid hormone cell transporter deficiency: a case report and review of the literature. Rev Neurol. 2013;56(12):615-622.

392. Masnada S, Groenweg S, Saletti V, et al. Novel mutations in SLC16A2 associated with a less severe phenotype of MCT8 deficiency. Metab Brain Dis. 2019;34(6):1565-1575.

393. Kersseboom S, Kremers G], Friesema EC, et al. Mutations in MCT8 in patients with AllanHerndon-Dudley-syndrome affecting its cellular distribution. Mol Endocrinol. 2013;27(5):801-813.

394. Friesema EC, Visser WE, Visser TJ. Genetics and phenomics of thyroid hormone transport by MCT8. Mol Cell Endocrinol. 2010;322(1-2):107-113.

395. Jansen J, Friesema EC, Kester MH, Schwartz CE, Visser T). Genotype-phenotype relationship in patients with mutations in thyroid hormone transporter MCT8. Endocrinology. 2008;149(5):2184-2190.

396. Matheus MG, Lehman RK, Bonilha L, Holden KR. Redefining the pediatric phenotype of $X$-linked monocarboxylate transporter 8 (MCT8) deficiency: implications for diagnosis and therapies. J Child Neurol. 2015;30(12):1664-1668.

397. Groeneweg S, Peeters RP, Moran C. Effectiveness and safety of Triac in children and adults with MCT8 deficiency: an international, multicentre, single group, open-label, phase 2 trial. Lancet Diabetes Endocrinol. 2019;7(9):695-706.

398. Dumitrescu AM, Refetoff S. Impaired sensitivity to thyroid hormone: defects of transport, metabolism and action. In: Feingold KR, Anawalt B, Boyce A, et al., eds. Endotext [Internet]. South Dartmouth, MA: MDText. com, Inc.; 2000-2015.

399. Moran C, Chatterjee K. Resistance to thyroid hormone $\alpha$-emerging definition of a disorder of thyroid hormone action. J Clin Endocrinol Metab. 2016;101(7):2636-2639.

400. Schwartz CE, Stevenson RE. The MCT8 thyroid hormone transporter and Allan-Herndon-Dudley syndrome. Best Pract Res Clin Endocrinol Metab. 2007;21(2):307-321.

401. Ramos HE, Morandini M, Carré A, et al. Pregnancy in women heterozygous for MCT8 mutations: risk of maternal hypothyroxinemia and fetal care. Eur J Endocrinol. 2011;164(2):309-314.

402. Constant EL, de Volder AG, Ivanoiu A, et al. Cerebral blood flow and glucose metabolism in hypothyroidism: a positron emission tomography study. J Clin Endocrinol Metab. 2001;86(8):3864-3870.

403. Cremer GM, Goldstein NP, Paris J. Myxedema and ataxia. Neurology. 1969;19(1):37-46.

404. Westerholz S, de Lima AD, Voigt T. Regulation of early spontaneous network activity and
GABAergic neurons development by thyroid hormone. Neuroscience. 2010;168(2):573-589.

405. Hashemi E, Ariza J, Rogers H, Noctor SC, MartínezCerdeño $V$. The number of parvalbumin-expressing interneurons is decreased in the prefrontal cortex in autism. Cereb Cortex. 2017;27(3):1931-1943.

406. Bernal J. Thyroid hormones and brain development. Vitam Horm. 2005;71:95-122.

407. Vaz FM, Paulusma CC, Huidekoper $H$, et al. Sodium taurocholate cotransporting polypeptide (SLC10A1) deficiency: conjugated hypercholanemia without a clear clinical phenotype. Hepatology. 2015;61(1):260-267.

408. Tan HJ, Deng $M$, Qiu JW, Wu JF, Song $Y Z$. Monozygotic twins suffering from sodium taurocholate cotransporting polypeptide deficiency: a case report. Front Pediatr. 2018;6:354.

409. Deng M, Mao M, Guo L, Chen FP, Wen WR, Song $Y Z$. Clinical and molecular study of a pediatric patient with sodium taurocholate cotransporting polypeptide deficiency. Exp Ther Med. 2016;12(5):3294-3300.

410. Liu R, Chen C, Xia X, et al. Homozygous p.Ser267Phe in SLC10A1 is associated with a new type of hypercholanemia and implications for personalized medicine. Sci Rep. 2017;7(1):9214.

411. Qiu JW, Deng $M$, Cheng $Y$, et al. Sodium taurocholate cotransporting polypeptide (NTCP) deficiency: identification of a novel SLC10A1 mutation in two unrelated infants presenting with neonatal indirect hyperbilirubinemia and remarkable hypercholanemia. Oncotarget. 2017;8(63):106598-106607.

412. Tărlungeanu DC, Deliu E, Dotter CP, et al. Impaired amino acid transport at the blood brain barrier is a cause of autism spectrum disorder. Cell. 2016;167(6):1481-1494.e18.

413. Di Cosmo C, Liao XH, Ye H, et al. Mct8-deficient mice have increased energy expenditure and reduced fat mass that is abrogated by normalization of serum T3 levels. Endocrinology. 2013;154(12):4885-4895.

414. Leitch VD, Di Cosmo C, Liao XH, et al. An essential physiological role for MCT8 in bone in male mice. Endocrinology. 2017;158(9):3055-3066.

415. Rodrigues TB, Ceballos A, Grijota-Martínez C, et al. Increased oxidative metabolism and neurotransmitter cycling in the brain of mice lacking the thyroid hormone transporter SLC16A2 (MCT8). PloS One. 2013;8(10):e74621.

416. Bárez-López S, Obregon MJ, Bernal J, GuadañoFerraz A. Thyroid hormone economy in the perinatal mouse brain: implications for cerebral cortex development. Cereb Cortex. 2018;28(5):1783-1793.

417. Ferrara $\mathrm{AM}$, Liao XH, Gil-lbáñez $\mathrm{P}$, et al. Changes in thyroid status during perinatal development of MCT8-deficient male mice. Endocrinology. 2013;154(7):2533-2541.

418. Wirth EK, Sheu SY, Chiu-Ugalde J, et al. Monocarboxylate transporter 8 deficiency: altered thyroid morphology and persistent high triiodothyronine/thyroxine ratio after thyroidectomy. Eur J Endocrinol. 2011;165(4):555-561.

419. Sharlin DS, Ng L, Verrey F, et al. Deafness and loss of cochlear hair cells in the absence of thyroid hormone transporters Slc16a2 (Mct8) and Slc16a10 (Mct 10). Sci Rep. 2018;8(1):4403.

420. Weber J, Mclnnes J, Kizilirmak C, et al. Interdependence of thyroglobulin processing and thyroid hormone export in the mouse thyroid gland. Eur J Cell Biol. 2017;96(5):440-456.

421. Espino Guarch M, Font-Llitjos M, Murillo-Cuesta S, et al. Mutations in L-type amino acid transporter-2 
support SLC7A8 as a novel gene involved in agerelated hearing loss. eLife. 2018;7:e31511.

422. Galton VA, Schneider MJ, Clark AS, St Germain DL. Life without thyroxine to 3,5,3'-triiodothyronine conversion: studies in mice devoid of the 5'-deiodinases. Endocrinology. 2009; 150(6):2957-2963.

423. Barez-Lopez S, Grijota-Martinez C, Auso E, et al. Adult mice lacking Mct8 and Dio2 proteins present alterations in peripheral thyroid hormone levels and severe brain and motor skill impairments. Thyroid. 2019;29(11):1669-1682.

424. Hernandez A, Martinez ME, Fiering S, Galton VA, St Germain D. Type 3 deiodinase is critical for the maturation and function of the thyroid axis. J Clin Invest. 2006;116(2):476-484.

425. Fliers E, Unmehopa UA, Alkemade A. Functional neuroanatomy of thyroid hormone feedback in the human hypothalamus and pituitary gland. Mol Cell Endocrinol. 2006;251(1-2):1-8.

426. Müller J, Heuer $H$. Understanding the hypothalamus-pituitary-thyroid axis in mct8 deficiency. Eur Thyroid J. 2012;1 (2):72-79.

427. Wirth EK, Rijntjes E, Meyer F, Köhrle J, Schweizer U. High T3, low T4 serum levels in Mct8 deficiency are not caused by increased hepatic conversion through type i deiodinase. Eur Thyroid J. 2015;4(Suppl 1):87-91.

428. Buitendijk M, Galton VA. Is the kidney a major storage site for thyroxine as thyroxine glucuronide? Thyroid. 2012;22(2):187-191.

429. Vancamp P, Darras VM. From zebrafish to human: a comparative approach to elucidate the role of the thyroid hormone transporter MCT8 during brain development. Gen Comp Endocrinol. 2018; 265:219-229.

430. Vancamp P, Deprez MA, Remmerie M, Darras VM Deficiency of the thyroid hormone transporter monocarboxylate transporter 8 in neural progenitors impairs cellular processes crucial for early corticogenesis. J Neurosci. 2017;37(48):11616-11631.

431. Vancamp P, Bourgeois NMA, Houbrechts AM, Darras VM. Knockdown of the thyroid hormone transporter MCT8 in chicken retinal precursor cells hampers early retinal development and results in a shift towards more UV/blue cones at the expense of green/red cones. Exp Eye Res. 2019;178:135-147.

432. Saunders NR, Liddelow SA, Dziegielewska KM. Barrier mechanisms in the developing brain. Front Pharmacol. 2012;3:46.

433. Landers K, Richard K. Traversing barriers-how thyroid hormones pass placental, blood-brain and blood-cerebrospinal fluid barriers. Mol Cell Endocrinol. 2017;458:22-28

434. Dratman MB, Crutchfield FL, Schoenhoff MB. Transport of iodothyronines from bloodstream to brain: contributions by blood:brain and choroid plexus:cerebrospinal fluid barriers. Brain Res. 1991;554(1-2):229-236

435. Chanoine JP, Alex S, Fang SL, et al. Role of transthyretin in the transport of thyroxine from the blood to the choroid plexus, the cerebrospinal fluid, and the brain. Endocrinology. 1992;130(2):933-938.

436. Schweizer U, Köhrle J. Function of thyroid hormone transporters in the central nervous system. Biochim Biophys Acta. 2013;1830(7):3965-3973.

437. Richardson SJ, Wijayagunaratne RC, D'Souza DG, Darras VM, Van Herck SL. Transport of thyroid hormones via the choroid plexus into the brain: the roles of transthyretin and thyroid hormone transmembrane transporters. Front Neurosci. 2015;9:66.
438. Richardson SJ, Lemkine GF, Alfama G, Hassani Z, Demeneix BA. Cell division and apoptosis in the adult neural stem cell niche are differentially affected in transthyretin null mice. Neurosci Lett. 2007:421(3):234-238.

439. Zhang $Y$, Chen $K$, Sloan $S A$, et al. An RNAsequencing transcriptome and splicing database of glia, neurons, and vascular cells of the cerebral cortex. J Neurosci. 2014;34(36):11929-11947.

440. Fleming A, Diekmann H, Goldsmith P. Functional characterisation of the maturation of the blood-brain barrier in larval zebrafish. PloS One. 2013;8(10):e77548

441. Jeong JY, Kwon HB, Ahn JC, et al. Functional and developmental analysis of the blood-brain barrier in zebrafish. Brain Res Bull. 2008:75(5):619-628.

442. Zhang Y, Sloan SA, Clarke LE, et al. Purification and characterization of progenitor and mature human astrocytes reveals transcriptional and functional differences with mouse. Neuron. 2016;89(1):37-53.

443. Calzà L, Baldassarro VA, Fernandez M, Giuliani A, Lorenzini L, Giardino L. Thyroid hormone and the white matter of the central nervous system: from development to repair. Vitam Horm. 2018; 106:253-281.

444. Schnell C, Shahmoradi A, Wichert SP, et al. The multispecific thyroid hormone transporter OATP1C1 mediates cell-specific sulforhodamine 101-labeling of hippocampal astrocytes. Brain Struct Funct. 2015;220(1):193-203.

445. Lang MF, Salinin S, Ridder DA, et al. A transgenic approach to identify thyroxine transporterexpressing structures in brain development. J Neuroendocrinol. 2011;23(12):1194-1203.

446. Dentice M, Marsili A, Zavacki A, Larsen PR, Salvatore D. The deiodinases and the control of intracellular thyroid hormone signaling during cellular differentiation. Biochim Biophys Acta. 2013;1830(7):3937-3945

447. Sharlin DS, Visser TJ, Forrest D. Developmental and cell-specific expression of thyroid hormone transporters in the mouse cochlea. Endocrinology. 2011;152(12):5053-5064

448. Vilches C, Boiadjieva-Knöpfel E, Bodoy S, et al. Cooperation of antiporter LAT2/CD98hc with uniporter TAT1 for renal reabsorption of neutral amino acids. J Am Soc Nephrol. 2018;29(6):1624-1635.

449. Gigena N, Alamino VA, Montesinos MD, et al. Dissecting thyroid hormone transport and metabolism in dendritic cells. J Endocrinol. 2017;232(2):337-350.

450. Guetg A, Mariotta L, Bock L, et al. Essential amino acid transporter Lat4 (Slc43a2) is required for mouse development. I Physiol. 2015;593(5):1273-1289.

451. Poncet N, Mitchell FE, Ibrahim AF, et al. The catalytic subunit of the system $L 1$ amino acid transporter (slc7a5) facilitates nutrient signalling in mouse skeletal muscle. PloS One. 2014;9(2):e89547.

452. Kang HJ, Kawasawa Yl, Cheng F, et al. Spatiotemporal transcriptome of the human brain. Nature. 2011;478(7370):483-489.

453. Bernal J. Thyroid hormone transport in developing brain. Curr Opin Endocrinol Diabetes Obes. 2011; 18(5):295-299.

454. Bernal J. Thyroid hormone receptors in brain development and function. Nat Clin Pract Endocrinol Metab. 2007;3(3):249-259.

455. Groeneweg S, Peeters RP, Visser TJ, Visser WE. Therapeutic applications of thyroid hormone analogues in resistance to thyroid hormone (RTH) syndromes. Mol Cell Endocrinol. 2017;458:82-90.
456. Groeneweg S, Visser WE, Visser TJ. Disorder of thyroid hormone transport into the tissues. Best Pract Res Clin Endocrinol Metab. 2017;31(2):241-253.

457. Filho HC, Marui S, Manna TD, et al. Novel mutation in MCT8 gene in a Brazilian boy with thyroid hormone resistance and severe neurologic abnormalities. Ara Bras Endocrinol Metabol. 2011;55(1):60-66.

458. Fu J, Refetoff S, Dumitrescu AM. Inherited defects of thyroid hormone-cell-membrane transport: review of recent findings. Curr Opin Endocrinol Diabetes Obes. 2013;20(5):434-440.

459. Rivkees SA, Mattison DR. Ending propylthiouracilinduced liver failure in children. N Engl J Med. 2009;360(15):1574-1575.

460. Di Cosmo C, Liao XH, Dumitrescu AM, Weiss RE, Refetoff S. A thyroid hormone analog with reduced dependence on the monocarboxylate transporter 8 for tissue transport. Endocrinology. 2009; 150(9):4450-4458.

461. Pennock GD, Raya TE, Bahl JJ, Goldman S, Morkin E. Cardiac effects of 3,5-diiodothyropropionic acid, a thyroid hormone analog with inotropic selectivity. J Pharmacol Exp Ther. 1992;263(1):163-169.

462. Ferrara $A M$, Liao $X H$, Ye $H$, Weiss RE, Dumitrescu AM, Refetoff S. The thyroid hormone analog DITPA ameliorates metabolic parameters of male mice with mct8 deficiency. Endocrinology. 2015;156(11):3889-3894

463. Ferrara $A M$, Liao XH, Gil-Ibáñez P, et al. Placenta passage of the thyroid hormone analog DITPA to male wild-type and Mct8-deficient mice. Endocrinology. 2014;155(10):4088-4093.

464. Groeneweg S, Peeters RP, Visser TJ, Visser WE. Triiodothyroacetic acid in health and disease. I Endocrinol. 2017;234(2):R99-R121.

465. Takeda T, Suzuki S, Liu RT, DeGroot LJ. Triiodothyroacetic acid has unique potential for therapy of resistance to thyroid hormone. J Clin Endocrinol Metab. 1995;80(7):2033-2040.

466. Messier N, Laflamme L, Hamann G, Langlois MF. In vitro effect of Triac on resistance to thyroid hormone receptor mutants: potential basis for therapy. Mol Cell Endocrinol. 2001;174(1-2):59-69.

467. Martínez L, Nascimento AS, Nunes FM, et al. Gaining ligand selectivity in thyroid hormone receptors via entropy. Proc Natl Acad Sci U S A. 2009;106(49):20717-20722.

468. Bárez-López S, Obregon MJ, Martínez-deMena R, Bernal J, Guadaño-Ferraz A, Morte B. Effect of triiodothyroacetic acid treatment in mct8 deficiency: a word of caution. Thyroid. 2016;26(5):618-626.

469. Visser WE, Heuer H, Visser TJ. Triiodothyroacetic acid treatment in MCT8 deficiency: a word of nuance. Thyroid. 2016;26(5):615-617.

470. Bárez-López S, Hartley MD, Grijota-Martínez C, Scanlan TS, Guadaño-Ferraz A. Sobetirome and its amide prodrug Sob-AM2 exert thyromimetic actions in Mct8-deficient brain. Thyroid. 2018;28(9):1211-1220.

471. Hartley MD, Banerji T, Tagge IJ, et al. Myelin repair stimulated by CNS-selective thyroid hormone action. JCl Insight. 2019;4(8):126329.

472. Wainwright CE, Elborn JS, Ramsey BW, et al; TRAFFIC Study Group; TRANSPORT Study Group. Lumacaftor-ivacaftor in patients with cystic fibrosis homozygous for Phe508del CFTR. N Engl J Med. 2015;373(3):220-231.

473. Ciechanover A, Kwon YT. Protein quality control by molecular chaperones in neurodegeneration. Front Neurosci. 2017;11:185. 
474. Cortez L, Sim V. The therapeutic potential of chemical chaperones in protein folding diseases. Prion. 2014;8(2):28938.

475. Mol JA, Visser TJ. Synthesis and some properties of sulfate esters and sulfamates of iodothyronines. Endocrinology. 1985;117(1):1-7.

476. Rooda SJ, Otten MH, van Loon MA, Kaptein E, Visser TJ. Metabolism of triiodothyronine in rat hepatocytes. Endocrinology. 1989;125(4):2187-2197.

477. Jayarama-Naidu R, Johannes J, Meyer F, et al A Nonradioactive uptake assay for rapid analysis of thyroid hormone transporter function Endocrinology. 2015;156(7):2739-2745.

478. Dong $H$, Wade MG. Application of a nonradioactive assay for high throughput screening for inhibition of thyroid hormone uptake via the transmembrane transporter MCT8. Toxicol in Vitro. 2017:40:234-242

479. Rathmann D, Rijntjes E, Lietzow J, Köhrle J. Quantitative analysis of thyroid hormone metabolites in cell culture samples using LC-MS/ MS. Eur Thyroid J. 2015;4(Suppl 1):51-58.

\section{Acknowledgments}

We dedicate this review to the memory of our dear colleague, mentor, and friend, Prof Theo J. Visser, who passed away suddenly in 2018. His contributions to the field of thyroidology, in particular to thyroid hormone transporter research, are difficult to overestimate. He was among the first to demonstrate that the cellular entry of thyroid hormone is a carrier-mediated process that requires cell membrane thyroid hormone transporter proteins. His subsequent work resulted in the discovery and characterization of various thyroid hormone transporters. In particular, the discovery of MCT8 as a specific thyroid hormone transporter and mutations therein associated with a human disorder opened new avenues and fueled many groups worldwide to pursue research in the thyroid hormone transport field. Prof Visser inspired many generations of scientists during his career. Not only his brilliant scientific ideas and insights, but also his charming personality and humor were known to everyone.

Financial Support: The authors would like to acknowledge funding support by the Sherman family (to W.E.V.), Eurostars (E!11378; to W.E.V.), Erasmus MC fellowship (to W.E.V.), Deutsche Forschungsgemeinschaft (SPP1629; to H.H.), and Federal Ministry of Education and Research (E-RARE "Thyronerve," to H.H.).

Additional Information

Disclosure Summary: The Erasmus Medical Centre (Rotterdam, the Netherlands) might receive royalties from Rare Thyroid Therapeutics (the manufacturer of Triac) in the future, dependent on any future commercialization. None of the authors have personal disclosures relevant to this work.

Correspondence: W. Edward Visser, MD, PhD, Department of Internal Medicine, Thyroid laboratory, Room 502, Dr. Molewaterplein 40, 3015 GD Rotterdam, the Netherlands.E-mail:w.e.visser@erasmusmc.nl.

Abbreviations: ABC, ATP-binding cassette; AHDS, Allan-Herndon-Dudley syndrome; APC, amino acid/ polyamine/organocation transporter; BAT, brown adipose tissue; BBB, blood-brain barrier; BCSFB, bloodcerebrospinal fluid barrier; $B M E C$, brain microvasculature endothelial; BSP, bromosulfophthalein; CNS, central nervous system; CRYM, mu-crystallin; CSFBB, cerebrospinal fluid brain barrier; DEPC, diethylpyrocarbonate; DHEAS, dehydroepiandrosterone sulfate; DIO, deiodinating enzyme; DIT, 3,5-di-iodotyrosine; DITPA, 3,5-diiodothyropropionic acid; E3S, estron-3-sulfate; GW, gestational week;HPT, hypothalamus-pituitary-thyroid; IMT, 3-iodo- $\alpha$-methyl-tyrosine; iPSCs, induced pluripotent stem cells; ISH, in situ hybridization; Km, Michaelis constant; Ko, knock-out; LAT, L-type amino acid transporters; L-dopa, L-3,4-dihydroxyphenylalanine; L-T4, levothyroxine; MCT, monocarboxylate transporter; MFS, major facilitator; MIT, 3-mono-iodotyrosine; NEM, N-ethylmaleimide; NPT, Na+-dependent phosphate transporters; NTCP, sodium/taurocholate cotransporting polypeptide; OATP, organic anion transporting polypeptide; PBA, phenylbutyric acid; PTU, propylthiouracil; PV, parvalbumin; PVN, paraventricular hypothalamic nucleus; RAR, retinoic acid receptor; RTH, resistance to thyroid hormone; SHBG, sex hormone-binding globulin; SLC, solute carrier; SNP, singlenucleotide polymorphism; T3, 3,5,3'-tri-iodothyronine; T4, thyroxine; Tetrac, tetraiodothyroacetic acid; TLS, translational start site; TMD, transmembrane domain; TRE, T3response elements; Triac, triiodothyroacetic acid; TRH, thyrotropin-releasing hormone; TSH, thyrotropin (formerly thyroid-stimulating hormone); TTR, transthyretin; $V$ GLUT, vesicular glutamate transporters; VNUT, vesicular nucleotide transporter; XPTC, X-linked PEST containing transporter. 